I $19.4 / 2: 872$

\title{
Reclamation of Mined Lands In the Western Coal Region
} GEOLOGICAL SURVEY CIRCULAR 872

\author{
DEPOSITORY \\ SKIDMOPE COLLEGE LIBRARY \\ REC'D JUN 61383 \\ SARATOGA SPRINGS, N.Y.
}

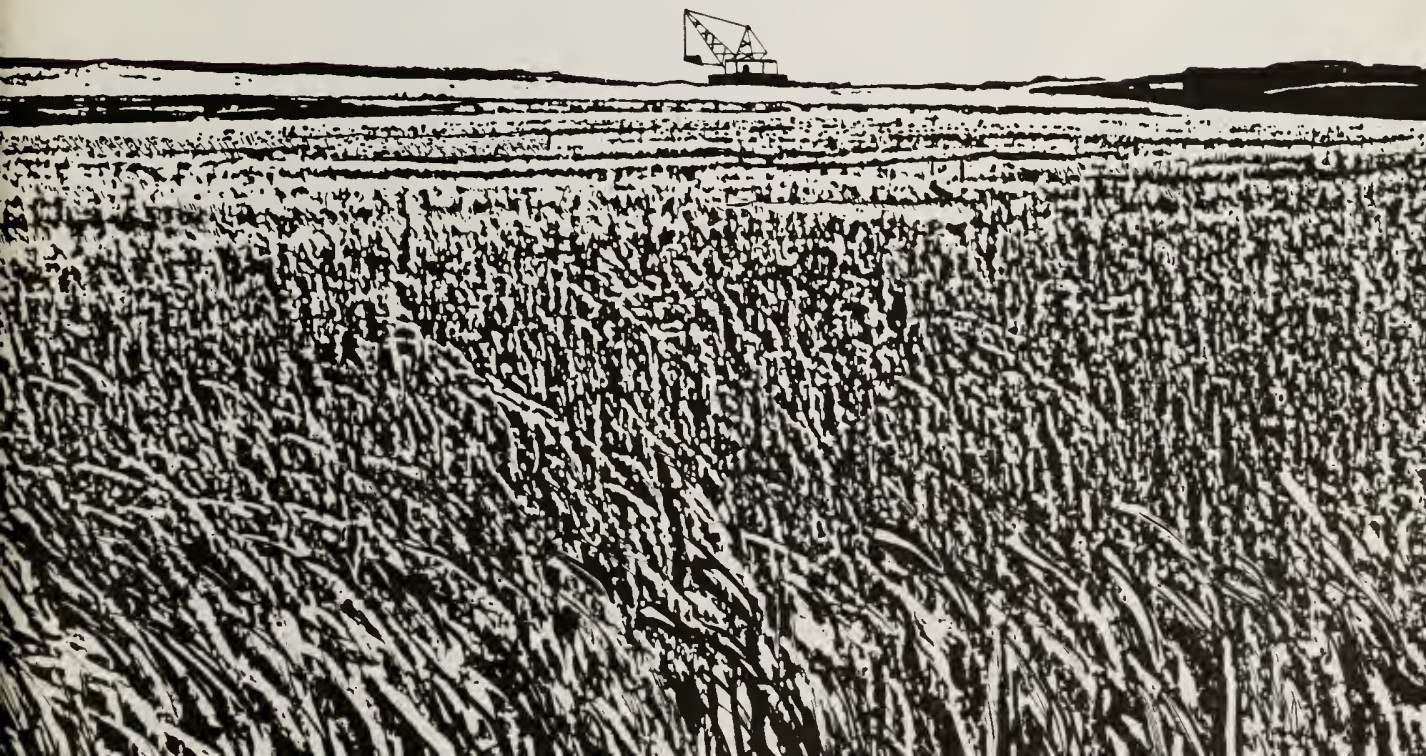




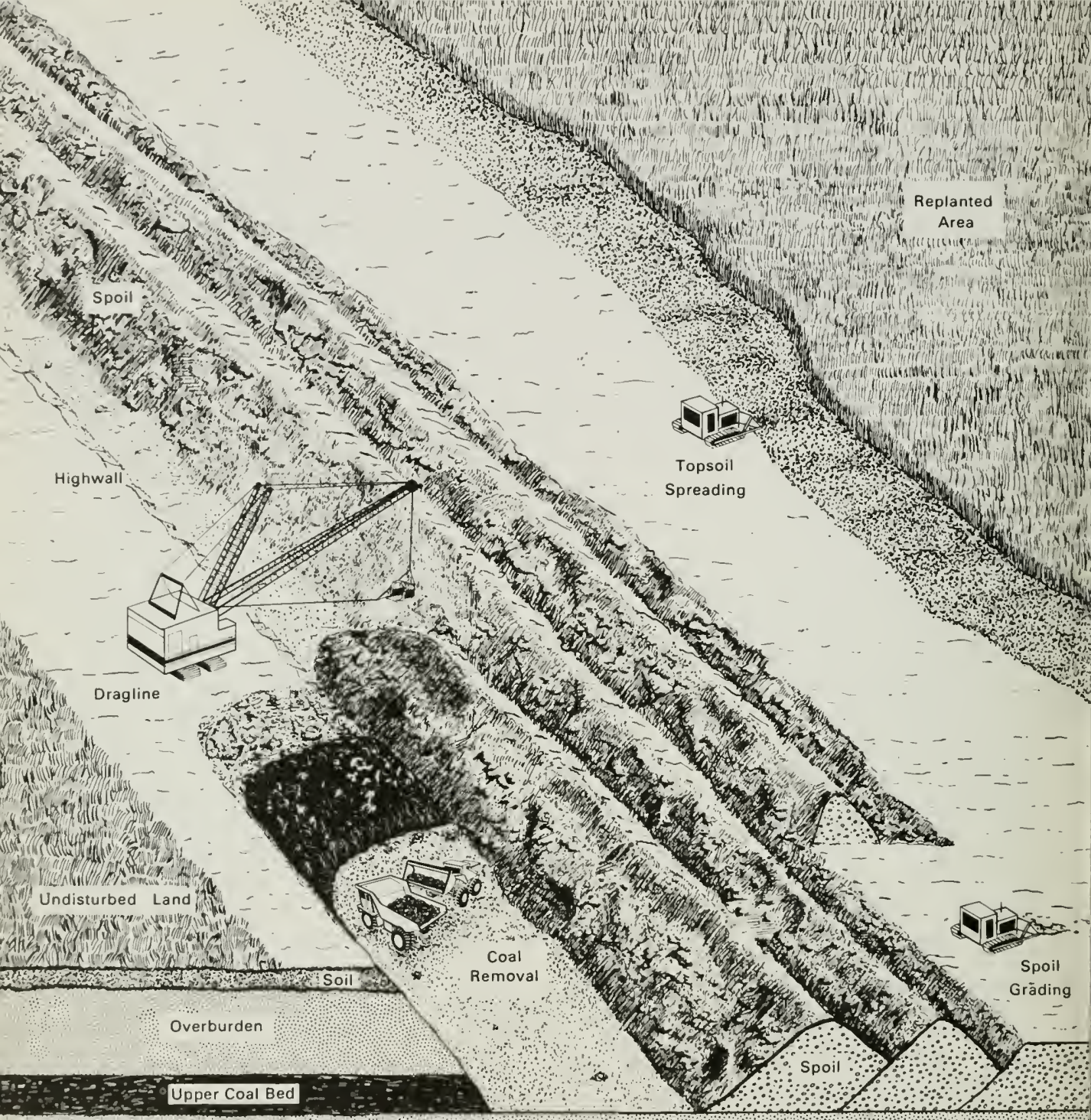

Interburden 
From the collection of the

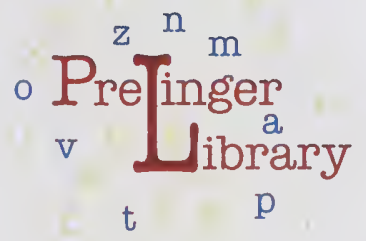

San Francisco, California 2008

\section{Reclamation of Mined Lands In the Western Coal Region}




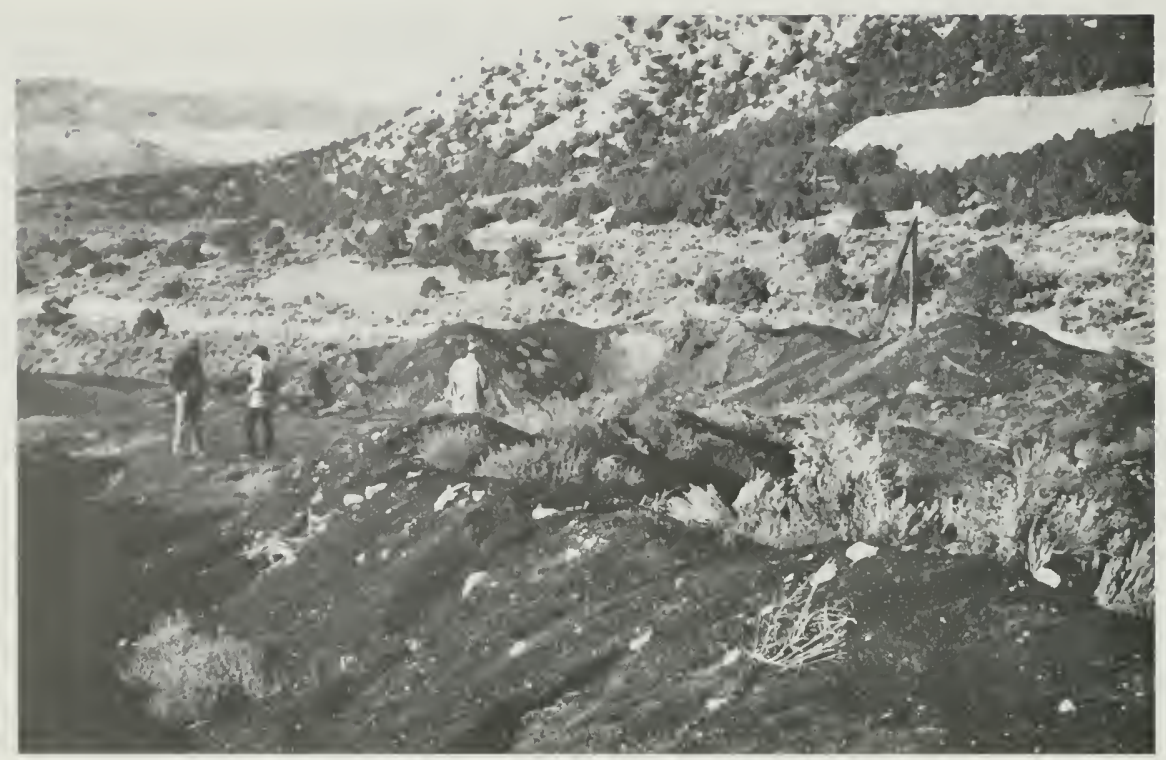

Loose coal waste at the old abandoned Weber mine in Utah. In more than 30 years, except on the north slopes, few plants have been able to establish themselves on these coal wastes because of high surface temperatures. The background shows typical mixed pinyon-juniper woodland and sagebrush lands with patches of grassland. The spoils have been left undisturbed; they lack grading, topsoiling, and seeding characteristics of later surfacemining operations. October 1978.

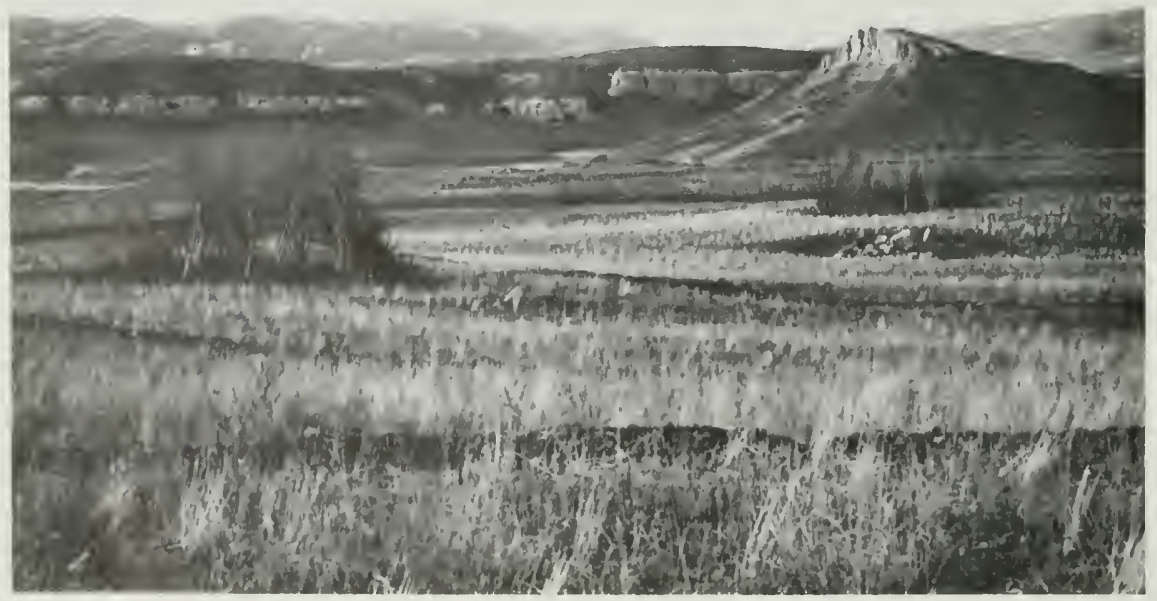

Reclamation at the Energy No. 2 mine in Colorado, showing transplanted aspen clumps, rock piles, and contour furrows on 2-year-old grassed slopes. Thls design aids in establishing a natural diversity of species and speeds up the reclamation process. November 1978. 


\section{Reclamation of Mined Lands in the Western Coal Region}

By Perry F. Narten, Stephen F. Litner, John W. Allingham, Lee Foster, Daniel M. Larsen and Harry C. McWreath, III

GEOLOGICAL SURVEY CIRCULAR 872 


\section{United States Department of the Interior}

JAMES G. WATT, Secretary

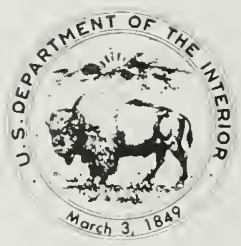

\section{Geological Survey}

Dallas L. Peck, Director

\section{Library of Congress Cataloging in Publication Data}

Main entry under title:

Reclamation of mined lands in the western coal region.

(Geological Survey circular ; 872)

Bibliography: $p$.

1. Coal mines and mining-Environmental aspects-

West (U.S.) 2. Reclamation of land-West (U.S.)

I. Narten, Perry F. II. Series.

QE75.C5 no. $872 \quad 557.3 \mathrm{~s}$ [333.73] 82-600118

[TD195.C58] 


\section{PREFACE}

This report is intended to provide readers from Federal, State, and local governments and the general public with a description of the process of mining and reclamation in the coalfields of the Western United States and some of the associated environmental problems. Reclamation is complex and involves many technical disciplines. The technical language in this report has been simplified as much as possible, or defined. The report includes photographs that illustrate various aspects of reclamation and some of the problems encountered in the reclamation process and a table that presents an overview of reclamation at 22 coal mines.

The report is based on 1978 observations by personnel of the Geological Survey, the Bureau of Land Management, and the Office of Surface Mining Reclamation and Enforcement of the U.S. Department of the Interior and of the Forest Service, U.S. Department of Agriculture. Our observations, individual and collective, have been supplemented by a review of the available literature. 
alfalfa

alkali sacaton -antelope bitterbrush

aspen

big bluegrass -

big sagebrush -..

bitterbrush --

black greasewood -

black sagebrush -.-_-_-_-

bluebunch wheatgrass --_-_-

blue grama --.--

bluegrass --

bluestem -.-

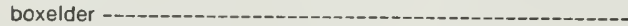

brome ----1-

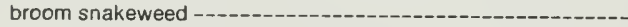

cereal rye --

cheatgrass -...---

Chinese elm --_.

chokecherry --

cottonwood -.---

crested wheatgrass --_-

fescue --

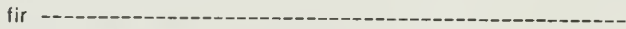

fourwing saltbush --_.

fringed sagebrush --

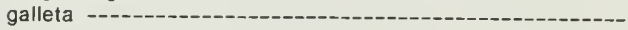

Gambel oak --

giant dropseed

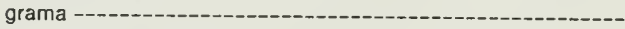

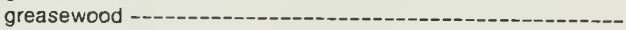

Great Basin wildrye -

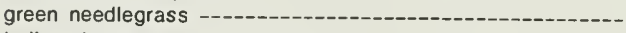

Indian ricegrass -----_-

intermediate wheatgrass

Japanese brome --_-_-_-

junegrass --

juniper --

little bluestem -.

millet --

Mormon tea ---

mountain brome -

mountain mahogany -

needleandthread -...--

oak --

orchardgrass --

pine (pinyon) -

ponderosa pine --_--_-

prairie sandreed --

prickly pear -

pubescent wheatgrass -.

rabbitbrush -_-

ricegrass --

rose - -

Russian-olive --_-

Russian-thistle --

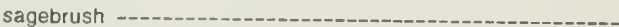

sainfoin

saltbush

saltgrass
Medicago sativa

Sporobolus airoides

Purshia tridentata

Populus spp.

Poa ampla

Artemisia tridentata

See antelope bitterbrush

Sarcobatus vermiculatus

Artemisia nova

Agropyron spicatum

Bouteloua gracilis

Poa spp.

Schizachyrium spp.

Acer negundo

Bromus spp.

Gutierrezia sarothrae

Secale cereale

Bromus tectorum

Uimus parviflora

Prunus virginiana

Populus spp.

Agropyron cristatum

Festuca spp.

Abies spp.

Atriplex canescens

Artemisia frigida

Hilaria jamesii

Quercus gambelii

Sporobolus giganteus

Bouteloua spp.

Sarcobatus vermiculatus

Elymus cinereus

Stipa viridula

Oryzopsis hymenoides

Agropyron intermedium

Bromus japonicus

Koeleria cristata

Juniperus spp.

Andropogon scoparius

Panicum miliaceum

Ephedra viridis

Bromus marginatus

Cerocarpus spp.

Stipa comata

Quercus spp.

Dactylis glomerata

Pinus edulis

Pinus ponderosa

Calamovilfa longifolia

Opuntia spp.

Agropyron trichophorum

Chrysothamnus spp.

Spartina spp.

Rosa spp.

Elaeagnus angustifolia

Salsola kali

Artemisia spp.

Onobrychis spp.

Atriplex spp.

Distichlis stricta 


\section{List of Common and Scientific Plant Names Used-Continued}

Sandberg bluegrass

sand dropseed

sandhill muhly

scrub oak -

sedge

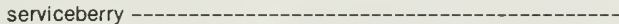

shadscale saltbush or shadscale

silver sagebrush

skunkbush

slender wheatgrass

smooth brome

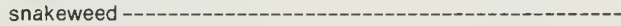

snowberry

spruce --

streambank wheatgrass

Sudan grass

summer-cypress

sweetclover ---

tall wheatgrass

thickspike wheatgrass

threadleaf sedge

western wheatgrass

white clover

willow

winter wheat

yellow sweetclover
Poa secunda

Sporobolus cryptandrus

Muhlenbergia pungens

Quercus spp.

Carex spp.

Amelanchier spp.

Atriplex confertifolia

Artemisia cana

Rhus trilobata

Agropyron trachycaulum

Bromus inermis

Gutierrezia spp.

Symphoricarpus oreophilus

Picea spp.

Agropyron riparium

Sorghum sudanense

Kochia lasiocarpa

Melilotus spp.

Agropyron elongatum

Agropyron dasystachyum

Carex filifolia

Agropyron smithii

Trifolium repens

Salix spp.

Triticum spp.

Melilotus officinalis 


\section{English-Metric Conversion}

\begin{tabular}{|c|c|c|}
\hline $\begin{array}{c}\text { MULTIPLY } \\
\text { ENGLISH UNITS }\end{array}$ & BY & $\begin{array}{l}\text { TO OBTAIN } \\
\text { METRIC UNITS }\end{array}$ \\
\hline $\begin{array}{l}\text { inches (in.) } \\
\text { feet (ft) } \\
\text { miles (mi) } \\
\text { acres } \\
\text { cubic yards }\left(\mathrm{yd}^{3}\right) \\
\text { pounds (lb) } \\
\text { pounds per acre (Ib/acre) } \\
\text { degrees Fahrenheit }\left({ }^{\circ} \mathrm{F}\right)\end{array}$ & $\begin{array}{l}2.54 \\
3.048 \times 10^{1} \\
3.048 \times 10^{-1} \\
1.609 \\
4.047 \times 10^{-1} \\
4.047 \times 10^{-3} \\
7.646 \times 10^{-1} \\
4.536 \times 10^{-1} \\
1.12 \\
5 / 9\left({ }^{\circ} \mathrm{F}-32\right)\end{array}$ & $\begin{array}{c}\text { centimeters }(\mathrm{cm}) \\
\text { centimeters }(\mathrm{cm}) \\
\text { meters }(\mathrm{m}) \\
\text { kilometers }(\mathrm{km}) \\
\text { hectares }(\mathrm{ha}) \\
\text { square kilometers }\left(\mathrm{km}^{2}\right) \\
\text { cubic meters }\left(\mathrm{m}^{3}\right) \\
\text { kilograms }(\mathrm{kg}) \\
\text { kilograms per hectare }(\mathrm{kg} / \mathrm{ha}) \\
\text { degrees Celsius }\left({ }^{\circ} \mathrm{C}\right)\end{array}$ \\
\hline
\end{tabular}




\section{CONTENTS}

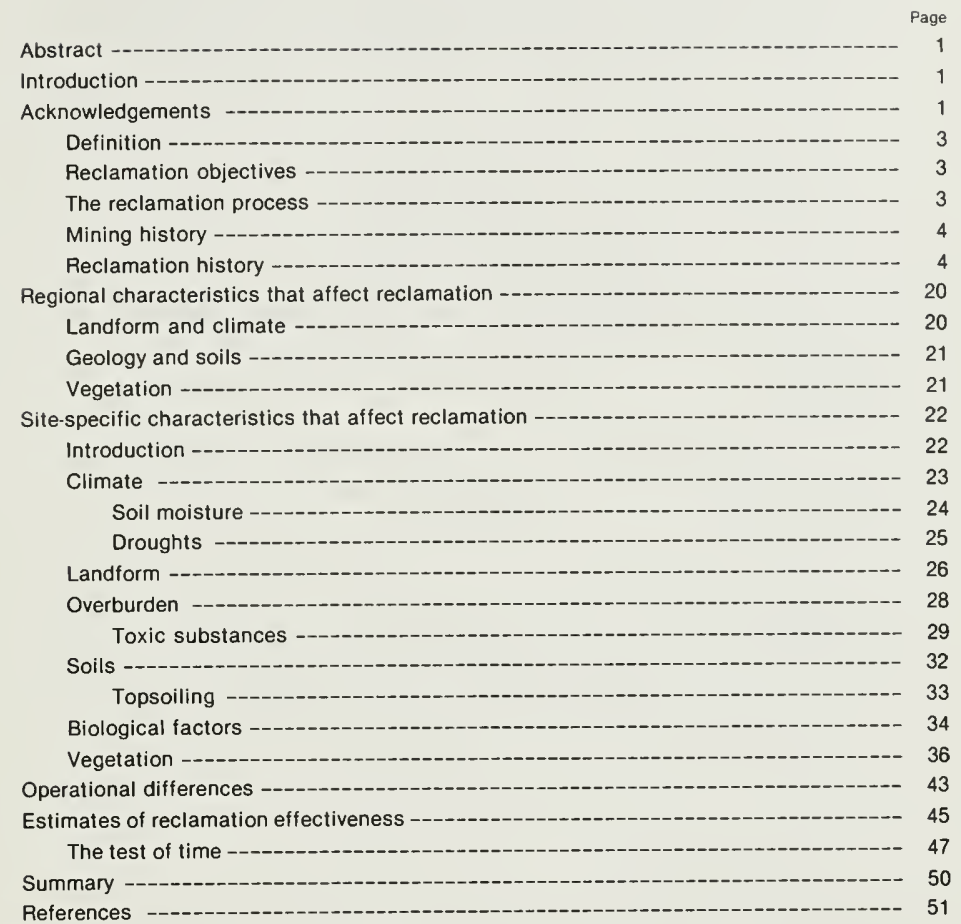

\section{ILLUSTRATIONS}

FRONTISPIECE. Photographs contrasting old abandoned Weber coal mine in Utah with recent reclamation methods used at the Energy 2 mine in Colorado

Figure 1. Map showing locations of 22 western coal mines assessed in the study -----2. Aerial photograph showing surface mine of the Arrowhead Coal Company near Haynes, North Dakota, and subsidence over the old abandoned underground mine -3. Photograph showing truck leaving a boxcut at the West Decker coal mine in Montana -- 6

4. Aerial photograph of the West Decker mine in Montana - 7

5. Aerial photograph showing the Navajo surface coal mine in New Mexico------ 20 
6. Photograph showing waste coal dumped below the entry of the abandoned Reminger coal mine in Utah -..-_-

7. Aerial photograph showing rows of abandoned spoil piles at the Rosebud mine in Montana -..--_...-

8. Photograph showing natural revegetation on the 19-year-old mine-waste dumps at the Elkol-Sorensen mine in Wyoming --_--_--

9. Photograph showing orphaned spoils naturally revegetated during the past 35 years at the Indian Head mine in North Dakota --

10. Photograph of revegetated orphaned spoils at the North Beulah mine in North Dakota

11. Photograph showing second season of revegetation by seeding at McKinley mine in New Mexico -

12. Photograph showing natural revegetation on spoil piles near the Seneca mines in Colorado --.-

13. Aerial photograph showing hummocky surface created by rounding off tops of spoils at an older part of the Seneca mine in Colorado -

14. Photograph showing graded and seeded spoil blending into the natural wooded terrain at the Absaloka mine in Montana --------

15. Map showing mean annual precipitation for the western coal region States ----

16. Map showing mean annual pan evaporation for the western coal region States -.-.....

17. Photograph showing grooved, mulched, and seeded slope at Seminoe 2 mine in Wyoming -

18. Photograph showing experimental water detention terraces at the San Juan mine in New Mexico ---_-

19. Aerial photograph showing mining and reclamation sequence at the Energy 1 mine in Colorado ---

20. Closeup photograph showing a contour furrow at the Energy 1 mine in Colorado -...-

21. Photograph showing a rough, ditched, and grooved reclaimed area at the McKinley mine in New Mexico -

22. Photograph showing a graded highwall at the Big Sky mine in Montana -------

23. Photograph showing eroded steep slope and natural revegetation of an old spoil pile along a haulroad at the Seminoe mines in Wyoming -...--

24. Photograph showing contrasting vegetation at the Indian Head mine in North Dakota

25. Map of soil series and associations in the Spring Creek permit area of Montana --.--

26. Photograph showing topsoil being spread on regraded spoil at Seminoe 2 mine in Wyoming -

27. Photograph showing sprinkler irrigation on topsoiled, graded spoils at the San Juan mine in New Mexico -.-..-

28. Photograph showing a soil column used for State certification in reclamation topsoiling at the Black Mesa mine in Arizona --

29. Photograph showing topsoil borrow strips at the Dave Johnston mine in Wyoming ------

30. Photograph showing densely revegetated slope of fourwing saltbush at the Navajo mine in New Mexico -..-

31. Photograph showing cattle grazing on young seedlings on a graded highwall at the Rosebud mine in Montana -

32. Photograph showing isolated 2- to 3-year-old saltbush plant on overgrazed reclaimed ground at the Black Mesa mine in Arizona -----------------

33. Photograph showing disturbed surface-mined land in various stages of revegetation at the Dave Johnston mine in Wyoming --_-_-_-

34. Closeup photograph of a small native ponderosa pine transplanted in the spring of 1978 at the Absaloka mine in Montana --

35. Aerial photograph showing mining and reclamation sequence at the Rosebud mine in Montana -

36. Photograph showing reclaimed area of wheatgrasses, pines, and junipers at the Big Sky mine In Montana --_-_-

37. Photograph showing rocks and a dead tree that provide perches for hawks and owls on reclaimed area at the Rosebud mine in Montana -.......-

21

22

23

24 
38. Photograph showing transplanted native aspens at the Energy 1 mine in

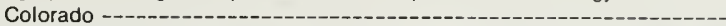

39. Photograph showing lush grasses on reclaimed land at the South Beulah mine in North Dakota

40. Photograph showing reclaimed land covered by grasses in their third growing season at the West Decker mine in Montana

41. Photograph showing grass-seeded reclaimed land blending into undisturbed land at the Vanguard mine in Wyoming

\section{TABLES}





\title{
Reclamation of Mined Lands in the Western Coal Region
}

\author{
By Perry F. Narten', Stephen F. Lintner', John W. Allingham', \\ Lee Foster ${ }^{2}$, Daniel M. Larsen ${ }^{2}$, and Harry C. McWreath III'
}

\begin{abstract}
In 1978, a group of scientists from several Federal agencies examined reclamation work at 22 coal mines in seven western States. The results of these examinations were not used to derive quantitative predictions of the outcome of reclamation work but rather to determine the general requirements for revegetation success. Locally, reclamation efforts are affected by climate, especially precipitation; the landform of the restored surface; the nature of the overburden material; the nature of the surface soil; and the natural ecological system. The goals of reclamation efforts are now broader than ever. Regulations call not only for reducing the steepness of the final surface and establishing a cover of mostly perennial native vegetation, but for restoring the land for specific land uses, achieving diversity both in types of plants and in number of species, and reintroduction of biological and ecological processes. If specific sites are monitored over a long enough period of time, quantitative predictions of success for individual mines may be possible, and such predictions can be included in environmental impact statements to help in the decisionmaking process. The results of this study indicate that current reclamation objectives can be met when the recla. mation effort is designed on the basis of site-specific needs and when existing technology is used.
\end{abstract}

\section{INTRODUCTION}

In October and November of 1978, scientists from the U.S. Geological Survey, the Bureau of Land Management, the Office of Surface Mining Reclamation and Enforcement, and the U.S. Forest Service visited 22 western coal mines at 17 locations in North Dakota, Montana, Wyoming, Colorado, Utah, Arizona, and New Mexico (fig. 1) to assess reclamation practices, to find out what had been accomplished and what was being done in planned reclamation, and to determine, if possible, what happens when minimal or

\footnotetext{
'Geological Survey, U.S. Department of the interior

${ }^{2}$ Forest Service, U.S. Department of Agriculture.
}

no attempts have been made at reclamation. These visits were made mostly to determine the outcome of revegetation work in respect to assessing environmental impacts of proposed coal mine development and forecasting reclamation success. Some observations were also made on techniques such as planting-site preparation and other factors that influence success.

The condition of land when it is abandoned after mining has been a major public concern and the focus of studies, laws, and regulations on reclamation. Regulatory agencies and the mining industry have made varied attempts at prescribing and implementing reclamation. This paper describes what is being done and delimits some predictions about the probable long-term effectiveness of present-day reclamation efforts in the western coal region.

To reach environmentally sound decisions on coal mine development, practical answers are needed to the following questions: Can selfsustaining diverse plant communities composed primarily of native species be reestablished? What will be the eventual vegetative production potentials of reclaimed land?

\section{ACKNOWLEDGMENTS}

The Federal team that conducted this study was assisted and guided by reclamation consultant Ed Nobel, who had participated in a 1976-77 detailed examination of plant and soil relationships on many of the same mines (Packer and others, 1981; Nobel, 1978a and b). Shirley Lindsay of the U.S. Office of Surface Mining and John Lovell of the U.S. Bureau of Land Management (now with the Office of Surface Mining), both with extensive experience in western coal 

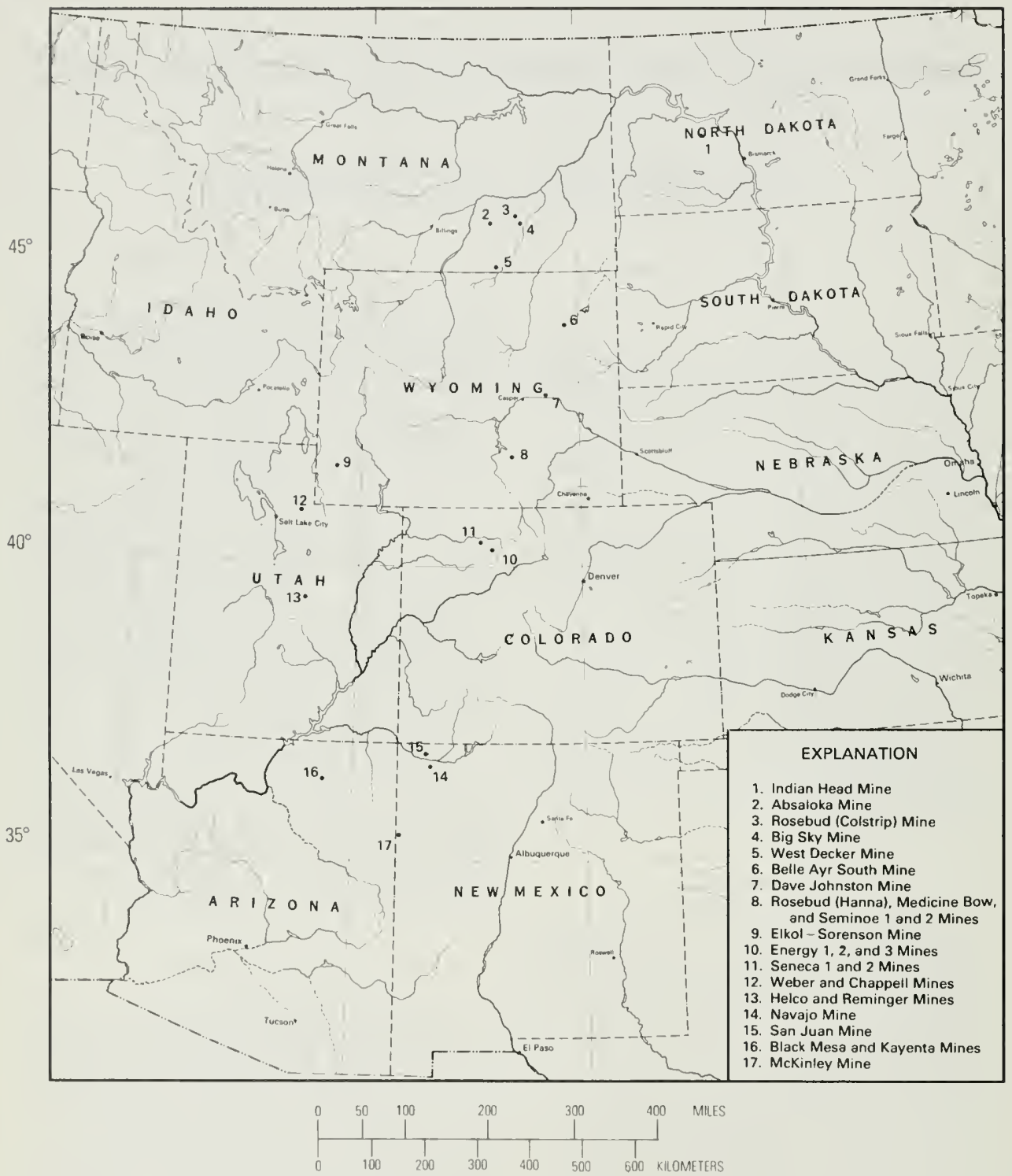

FIGUAE 1.-Locations of 22 western coal mines assessed in the study. 
mining reclamation problems, participated in the mine examinations in Utah, Colorado, New Mexico, and Arizona and contributed to this report in consultive and review capacities. The technical advice and logistical assistance of local Federal officials is gratefully acknowledged. These officials include Arnie Matilla, John Paul Storrs, Bill Newby, Vernon Ruill, Douglas Hileman, Archie Carver, Jim Edward, Bill Clarke, and Moon Hom of the U.S. Geological Survey, and James Webb, Richard Enriquez, Bryant Christenson, Jim Forest, Bob Black, and Ruby Hartman of the U.S. Forest Service.

Officials of the mining companies were particularly helpful in providing on-site guidance and discussion on their reclamation practices. They include Nick Bettas, Kemmerer Coal Company; Jerry Ellis, Rosebud Coal Company; Glen Doss, Gordon Peters, and Duane Richards, Pacific Power and Light; John Bradley, Amax Coal Company; Dave Jennings and Dwight Layton, Decker Coal Company; Reg Hoff, Walter BeGay, Jan Macfarlan, and Roy Karo, Peabody Coal Company; Ralph Moore and Bill Sullivan, Westmoreland Resources; Joe Mitzel, Jim Brown, and Terry Dudley, North American Coal Company; Jack Etherton, Al Czarnosky, Jr., Bill Noud, and Kent Crofts, Energy Fuels Corporation; Rodney Gabehart, Western Coal Company; and Sterling Grogan, Utah International and Midway Coal Mining Company.

\section{DEFINITION}

Planned reclamation, as defined in this report, is the process of returning mined lands to their premining productive capability or to a greater productive capability. It involves reshaping the disturbed lands into stable landforms and establishing a self-sustaining vegetative cover.

The majority of western Federal coal lands are classified as rangelands, which are used and managed as both wildlife habitats and as stock grazing areas. If a decision is made to return such lands to the same use after mining, it is implicit that the final result, the "reclaimed lands," will have (1) soil and plants established at about the same point in the plant succession, and (2) a plant diversity that is both self-sustaining under natural extremes of weather and climate and that provides a habitat for a variety of wildlife.
These goals commonly demand the use of a large component of native plants. The significant criterion of reclamation effectiveness, however, would be the restored capability and stability of the mined area as rangeland rather than the restoration of the premining ecosystem.

\section{RECLAMATION OBJECTIVES}

The objectives of reclamation have been changed over the years-between regions, between mines, and even within single mines-as the need for these changes became apparent to lawmakers. Contemporary goals in the western coal region have evolved to include (1) reducing the steepness of the land slopes on excavated materials, or "spoils," that overlay the mined coalbeds and of the end-of-mining cliff-like highwalls, and (2) establishing a cover of perennial native vegetation that can provide food and cover for livestock and wildlife and reduce the erosion potential. Initial implementation of these objectives has sometimes resulted in large areas that are nearly covered by a single nonnative legume or grass species or at most a few species. In recent years, because of greater awareness and concern for wildlife habitat and the possible long-term instability of monoculturelike plantings and nonnative plants, postmining efforts have been directed toward establishment of much more diverse plant communities and greater use of native plants, including woody browse shrubs and trees. The Surface Mining Control and Reclamation Act of 1977 (Public Law 95-87) has unified and broadened the objectives of reclamation of surface-mined lands on a nationwide basis.

\section{THE RECLAMATION PROCESS}

In the western surface coal mine region, reclamation is a continuing process, tied into the mining process, commencing with the initial removal and stockpiling of the topsoils. As mining progresses, the material above the coal, the overburden, is sequentially removed and deposited as "spoil piles" in previously minedout sections (see frontispiece). Any overburden materials usable for plant growth media may be salvaged and stockpiled at this time. The spoil 
piles are then graded, and topsoil or other suitable growth media are applied. The resulting surface is prepared for planting and is planted during the appropriate season. It may be treated in various ways before or after planting to improve germination and plant growth potentials, to reduce erosion, and to improve its water retention characteristics. In many mining operations, after the initial opening of the mine, the plant growth media can usually be placed directly on the graded surfaces without intermediate stockpiling.

Although the overall approaches to mining and reclamation are similar, local procedures will vary with differences in the attitude of the coalbeds (flat to steeply dipping); the number of individual beds to be mined; the thickness, strength, and mechanical and chemical properties of the materials above and between the coalbeds; and the types of equipment used. The costs of coal recovery and of handling overburden material directly influence the methods used. The technical aspects related to earthmaterial handling have reached an advanced state, and computer-assisted planning and decisionmaking models are now available (Scott, 1978; Gibson and others, 1979).

The revegetation process, though dependent on climate, the availability of suitable thicknesses of growing media, and of sources of plant materials, has been extensively researched for western coal surface-mined lands in recent years by the Surface Environment and Mining Program ${ }^{3}$ and other Federal and State government agencies and universities. In general, the same horticultural principles that are used for successful farming, home gardening, or landscaping, were found to apply in the revegetation process.

\section{MINING HISTORY}

Early western coal mines, which date back to the late 1800's, were usually small underground mines that served local markets and railroads. Subsidence has occurred over some of these underground mines creating surfaces that are hazardous and virtually unreclaimable (fig. 2).

\footnotetext{
${ }^{2}$ The Surface Environment and Mining Program (SEAM) was established by the U.S. Forest Service in 1973 and given a 5-year misston to develop and apply the most current reclamation end planning technology to western coal mining operations.
}

The subsidence is more common over mines with less overburden. In North Dakota, for example, overburdens of 100 feet or less have collapsed over several underground mines; those of 200 feet or more have not (Trimble, 1979, p. 16).

Early surface mining was restricted to a few large mines generally adjacent to railroads or powerplants. One of the oldest surface operations is the Rosebud mine at Colstrip, Montana, where mining began in 1924. Some of the surface mines in North Dakota are of similar age. The Indian Head mine, for example, began operating in 1929.

With the increased demand for electric power and low-sulfur coals and with the improvement of surface-mining machinery and transportation equipment (fig. 3), western coal mining began a period of major new development in the late 1960's and early 1970's. This boom has continued at a rapid pace, and some new surface mines are excavating the land surface at a high rate (fig. 4). For example, the Navajo mine in northwestern New Mexico disturbed about 4,500 acres (more than $7 \mathrm{mi}^{2}$ ) in 14 years (fig. 5). Environmental concerns about the reclamation of mined land have intensified as the rate of mining and the number of western surface mines has increased.

\section{RECLAMATION HISTORY}

Planned reclamation was not required and therefore not practiced in early western mining. Most mines were small and underground, and thus only a few surface acres were disturbed. In some areas, postmining debris such as surface buildings and load-out platforms have been removed by scavengers or land management agencies.

Only lump coal was marketable in early mining days, and the fine materials were sifted or washed out and left at the mine site. At many of these old abandoned underground mines, broken timbers and the coal fines and dust that blacken hillsides near the mine entrances are the major remaining physical indications of past mining. These blackened surfaces absorb enough heat to destroy any germinating seedlings and are largely devoid of vegetation (fig. 6).

In the early surface mines the overburden was left as it was excavated, generally in a series of small ridges having steep slopes (figs. 7 and 8 ), 


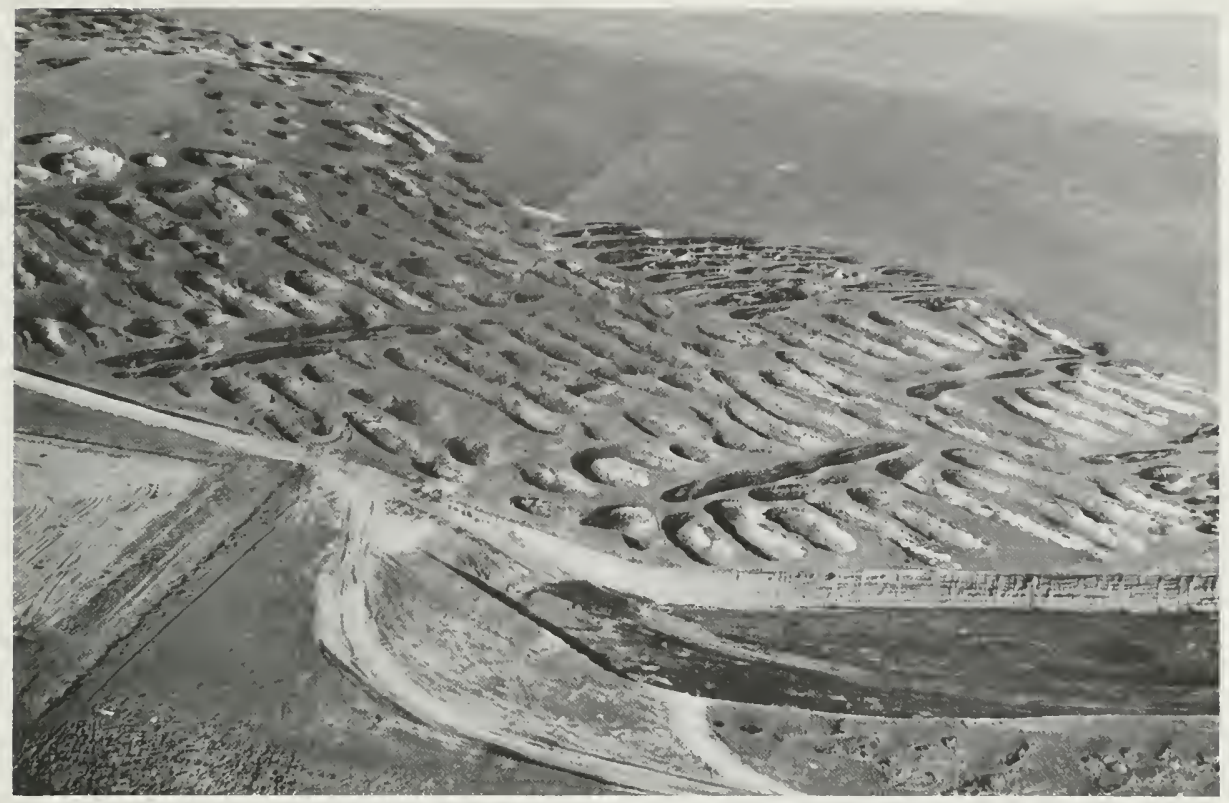

FIGURE 2.-Aerial photograph of the surface mine of the Arrowhead Coal Company in North Dakota, showing subsidence over the old abandoned underground mine workings in the background (Trimble, 1979). The highwall of the surface mine is 15 to 20 feet (foreground). Subsidence over underground room-and-pillar mines creates hazardous surfaces and destroys agricultural land; it does, however, create a local wildlife habitat. Such areas are difficult or impossible to reclaim.

to which fines from the coal were added. These ridges, commonly called orphaned spoils, provide examples of the natural revegetation process under the most adverse local conditions of moisture depletion, temperature stress, and soil instability.

In North Dakota where adequate moisture is available, some orphan spoils have, over a period of 40 years, become naturally revegetated with a variety of plant forms, mainly perennials. These plants have formed islands of vegetation in the mined lands and provide refuges for wildlife, especially deer (figs. 9 and 10). In some places, sportsmen and conservation groups have planted woody vegetation on spoil piles specifically to create wildlife refuges, and the vegetation is doing well (fig. 10). Canadian experience in converting old spoil piles to wildlife refuges has been similar (Schumacher and others, 1977, p. 19).
Most steep slopes in western coal mined areas, however, are generally bare of vegetation. Elsewhere vegetation is restricted to the most favorable germination sites, namely those where the soil surface is flat enough or where benches allow enough moisture to accumulate to support seed germination. Except in the more humid areas, plant cover is sparse even though some native plants are beginning to colonize (fig. 11). In some locations, moisture-collecting places between the spoil piles and at the bases of old highwalls and ponds are colonized by willows and cottonwoods which may locally dominate an otherwise barren landscape (fig. 7).

Some mining companies attempted to stabilize raw spoil piles by aerial seeding with either grasses or legumes, or both. Some State regulations required the leveling of the ridges (figs. -12 and 13). These newly leveled surfaces were then partly seeded from the air and, in places, were 


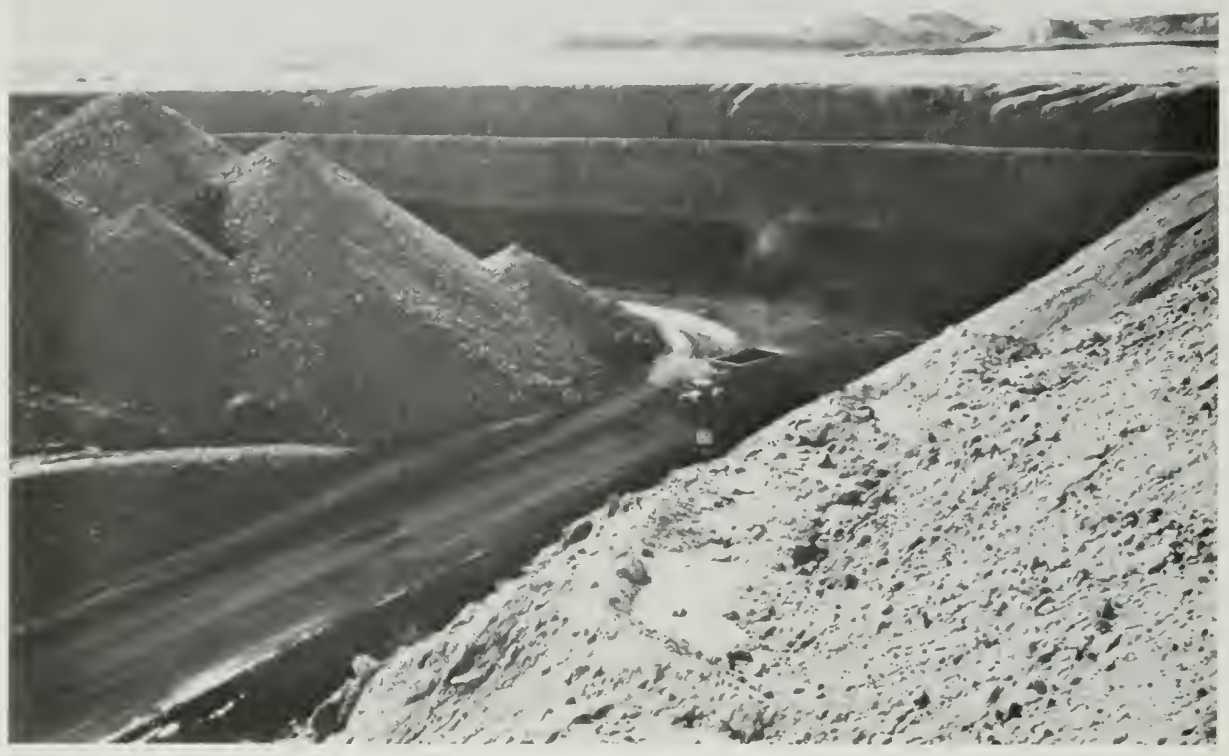

Figure 3.-A 100-ton truck leaves a box cut at the West Decker mine in Montana. Light-colored sandy overburden, about 90 feet thick, is stripped on two benches (away from the viewer) leaving spoil piles (left). Overburden will be stripped from the flat plain (center) as far as the distant buttes (background). The 52-foot coalbed is exposed in the box cut. September 1975.

planted with mostly nonnative trees and shrubs on an experimental basis in cooperation with State and Federal agencies (Berg, 1975, p. 82-83). Although evidence of deliberate seeding attempts can be inferred from the existence of nonnative grasses, the history is incomplete.

Recent reclamation efforts have been guided by both existing and proposed Federal and State regulations (Imhoff and others, 1976). In the 1970 's, government agencies, working in part with the universities, developed much of what is presently known about western reclamation technology. The state of the art is now believed sufficiently established that a major Federal program of the U.S. Forest Service, the Surface Environment and Mining Program (SEAM), changed its emphasis from research and development to assuring that reclamation technology is avail. able to users (Scholz, 1978). Much of the result of the SEAM program and other similar government and university research work (Burbank, 1978;
Brown, 1977) has been applied at one or more mines through experimental techniques and plantings done in cooperation with the mining companies. The mining companies are doing some testing and experimenting on their own (Deveraux, 1978) as new techniques are developed or as they are mandated by government regulation.

In 1977, the Surface Mining Control and Reclamation Act (SMCRA) was passed by Congress. This law called for the creation of a new Federal regulatory agency, The U.S. Office of Surface Mining Reclamation and Enforcement (OSM), whose principle duties include (1) administering the programs for controlling surface coal mining operations; (2) reviewing and approving or disapproving State programs for controlling mining operations and reclaiming mined lands; (3) inspecting for regulatory compliance; (4) issuing cease-and-desist orders; (5) ordering the supervision or revocation of any permit that does not 


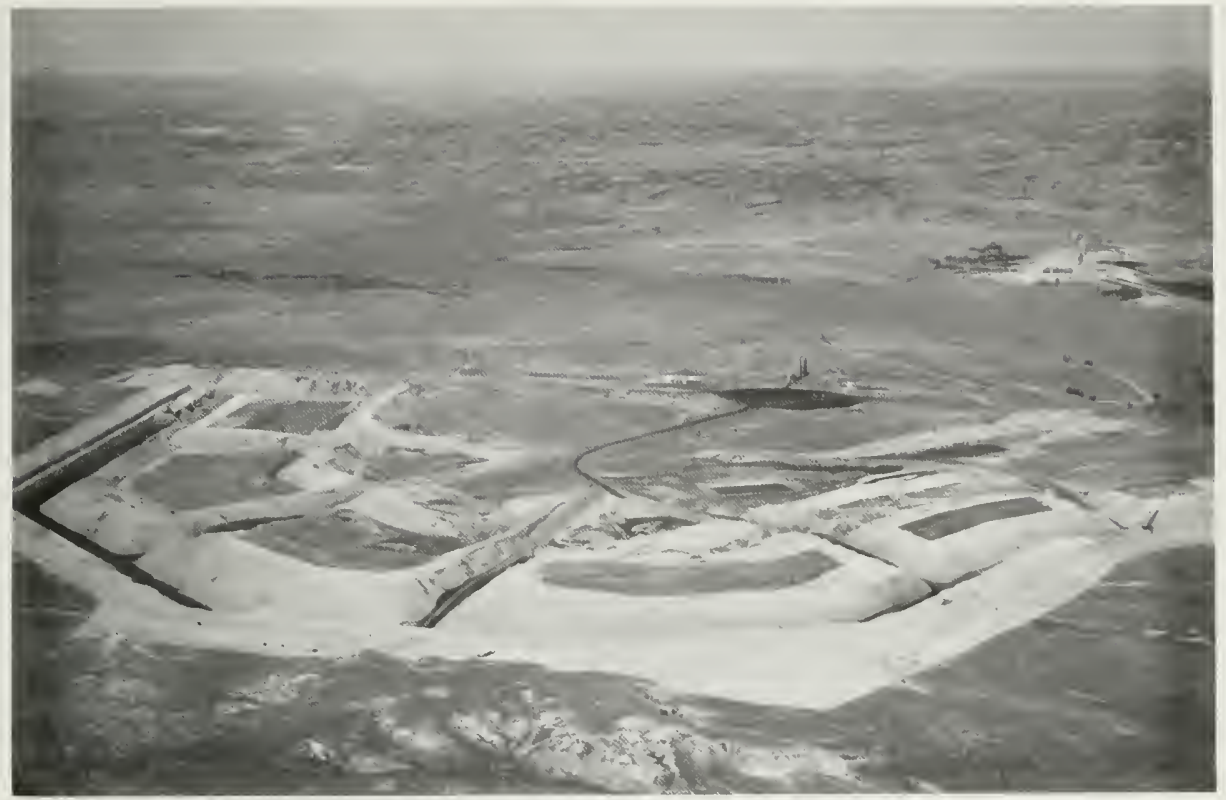

FIGURE 4.-Aerial photograph of the West Decker coal mine in Montana, adjacent to the Tongue River Reservoir. Systematic mining operations proceed radially outward. Regraded grass-covered reclaimed areas near the center are interrupted by radially oriented haulroads in deep cuts. Spoil is continuously regraded and seeded after the overburden has been stripped from the 52-foot coalbed. Irrigation is not needed, and grasses are quickly established. Extensive reclamation experiments have been made here under the SEAM Program of the U.S. Forest Service. Some replanted areas have multiple test plots. The East Decker Mine is across the reservoir at right center. July 1978.

comply with SMCRA or its regulations; (6) administering the program for the purchase and reclamation of abandoned and unreclaimed mined areas; and (7) assisting and monitoring programs for the research and development of improved surface mining and reclamation techniques designed to reduce adverse environmental and social effects.

An essential feature of the SMCRA was the call for cooperative development of legislation and technical organizations at the State level to support enforcement of Federal Government regulations. The Office of Surface Mining's regulations covering surface coal mining and reclamation operations were published on March 13, 1979 (U.S. Office of Surface Mining, 1979).

Although practices at individual mines may differ, reclamation now follows this basic scheme: the rough spoil is graded to approximate the original landform conforming to adjacent unmined terrain (fig. 14); the salvaged topsoil is respread; the surface is prepared as a seed bed, and measures are taken to supply whatever is necessary or required for good seedling development; the surface is planted; and post-planting amendments are made as needed.

At the time of the 1978 examinations of reclaimed areas, most of the revegetated mined lands examined were the result of reclamation initiated a few years earlier (see table 1). Some revegetation less than 5 years old was done in accordance with the general goals of recent legislation. The specific mandates of SMCRA for diversity of native communities, productivity, and cover, however, had not yet been met.

Any predictions about meeting current goals of reclamation are severely hampered by time 
The data are compiled from Evans, Uhlemann, and Eb8, 1978: Nobel, 1978a; Nobel, 1978b; field

\begin{tabular}{|c|c|c|c|c|c|}
\hline Misne and location & $\begin{array}{l}\text { Mining } \\
\text { Bcyinning date } \\
\text { Acres mined }\end{array}$ & $\begin{array}{l}\text { Reclamation } \\
\text { Begnning date } \\
\text { Acres reclaumed }\end{array}$ & Land Use & Climatic Factors & $\begin{array}{c}\text { Topography } \\
\text { Elevation, in feet }\end{array}$ \\
\hline $\begin{array}{l}\text { 1. INDIAN HEAD MIINE } \\
\text { Beulah, Mercer County, } \\
\text { central North Dakota } \\
\text { USGS } 7 y^{\prime} 2^{\prime} \text { maps: } \\
\text { Nedicine Butte NE } \\
\text { Nledicine Butte } \\
\text { Zap } \\
\text { Beulah } \\
\text { lat } 47^{\circ} 15^{\prime} \mathrm{N} . \\
\text { long } 101^{\circ} 52^{\prime} \mathrm{W} . \\
\text { secs. } 29-33, \mathrm{~T} .144 \mathrm{~N} \text {., } \\
\text { R. } 89 \mathrm{~W} \text {. }\end{array}$ & $\begin{array}{l}1929 \\
1,082 \text { acres } \\
\text { through } \\
1978 .\end{array}$ & $\begin{array}{l}\text { Unknown. } \\
608 \text { acres through } \\
1978 .\end{array}$ & $\begin{array}{l}\text { Premining: } \\
\text { Pasture, hay, } \\
\text { grains. } \\
\text { Intended: No } \\
\text { Change. } \\
\text { Livestock and } \\
\text { hunting ex- } \\
\text { cluded dur- } \\
\text { ing reclama- } \\
\text { tion. }\end{array}$ & $\begin{array}{l}\text { Growing season } \\
\text { (avg frost-free days): } 120 \text {. } \\
\text { Temperature }\left({ }^{\circ} \mathrm{F} \text { ): }\right. \\
\text { mean annual: } 41 \\
\text { range: }-40 \text { to } 110 \text {. } \\
\text { Precipitation (inches): } \\
\text { mean annual: } 17 \\
\text { range: } 11 \text { to } 22 ; \\
70 \text { percent from April } \\
\text { through August. }\end{array}$ & $\begin{array}{l}\text { Hilly with } 4 \text { - to 6-per- } \\
\text { cent slopes. Orphaned } \\
\text { spoils left from older } \\
\text { mining. Recent spoils } \\
\text { reshaped to original } \\
\text { forms. Surfaces } \\
\text { mostly stabilized even } \\
\text { on orphaned spoils. } \\
1,950-2,000\end{array}$ \\
\hline $\begin{array}{l}\text { 2. ABSALOKA MINE } \\
\text { Hardin, Big Horn Coun- } \\
\text { ty, southeastem } \\
\text { Montana } \\
\text { USGS } 7 y_{2}^{\prime} \text { maps: } \\
\text { Wolf School } \\
\text { lat } 45^{\circ} 48^{\prime} \mathrm{N} \text {.; } \\
\text { long } 107^{\circ} 05^{\prime} \mathrm{W} \\
\text { secs. } 23-26, \mathrm{~T} .37 \mathrm{~N} \text {., } \\
\text { R.1 E. }\end{array}$ & $\begin{array}{l}1974 \\
516 \text { acres } \\
\text { through } \\
1978 .\end{array}$ & $\begin{array}{l}1974 \\
253 \text { acres graded; } \\
127 \text { acres } \\
\text { seeded. }\end{array}$ & $\begin{array}{l}\text { Premining: } \\
\text { Wildlife and } \\
\text { livestock } \\
\text { range. } \\
\text { Intended: No } \\
\text { change. } \\
\text { Livestock and } \\
\text { hunting } \\
\text { excluded } \\
\text { during } \\
\text { reclamation. }\end{array}$ & $\begin{array}{l}\text { Growing season } \\
\text { (avg frost-free days): } 125 \\
\text { mid-May to mid- } \\
\text { September. } \\
\text { Temperature }\left({ }^{\circ} \mathrm{F}\right) \text { : } \\
\text { mean annual: } 46 \\
\text { range: }-42 \text { to } 100 \text {. } \\
\text { Precipitation (inches): } \\
\text { mean annual: } 12 \\
\text { range: } 2 \text { to } 15 ; \\
\text { mostly from May to } \\
\text { June. }\end{array}$ & $\begin{array}{l}\text { Rolling hills and buttes } \\
\text { with } 2 \text { - to } 8 \text {-percent } \\
\text { slopes. Some minor } \\
\text { rilling and gullying. } \\
3,500\end{array}$ \\
\hline $\begin{array}{l}\text { 3. ROSEBUD MINE } \\
\text { Colstrip. Rosebud Coun- } \\
\text { ty, southeastem } \\
\text { Montana } \\
\text { USGS } 7 y^{\prime} 2^{\prime} \text { maps: } \\
\text { Colstrip W. } \\
\text { Colstrip SW. } \\
\text { Colstrip SE. } \\
\text { Colstrip E. } \\
\text { lat } 45^{\circ} 52^{\prime} \text { N.; } \\
\text { long } 106^{\circ} 37^{\prime} \text { W. } \\
\text { T.I N., R. } 41-42 \mathrm{E} \text {. }\end{array}$ & $\begin{array}{l}1924-58 \\
1,800 \text { acres } \\
\text { mined by } \\
\text { Northern } \\
\text { Pacific RR. } \\
1968-79 \\
3,000 \text { total } \\
\text { acres dis- } \\
\text { turbed by } \\
\text { Western } \\
\text { Energy Co. }\end{array}$ & $\begin{array}{l}1969-74 \\
1,100 \text { acres } \\
\text { graded and } \\
\text { seeded by } \\
\text { Northern } \\
\text { Pacific RR; } \\
350 \text { acres re- } \\
\text { claimed by } \\
\text { Western } \\
\text { Energy Co.; } \\
350 \text { acres } \\
\text { abandoned. } \\
1975-78 \\
1,250 \text { acres } \\
\text { graded and } \\
\text { seeded. } \\
1979 \\
400 \text { acres graded } \\
\text { in preparation } \\
\text { for seeding. }\end{array}$ & $\begin{array}{l}\text { Premining: } \\
\text { Wildlife and } \\
\text { livestock } \\
\text { range; some } \\
\text { hayfields. } \\
\text { Intended: No } \\
\text { change. } \\
\text { Livestock ex- } \\
\text { cluded dur- } \\
\text { ing reclama- } \\
\text { tion. }\end{array}$ & $\begin{array}{l}\text { Growing season } \\
\text { (avg frost-free days): } 127 \\
\text { mid-May to mid- } \\
\text { September. } \\
\text { Temperature ( }{ }^{\circ} \mathrm{F} \text { ): } \\
\quad \text { mean annual: } 47 \\
\text { range: }-40 \text { to } 110 \text {. } \\
\text { Precipitation (inches): } \\
\text { mean annual: } 15 \\
\text { range: } 14 \text { to } 16 ; \\
60 \text { percent from March } \\
\text { through June. }\end{array}$ & $\begin{array}{l}\text { Sandstone ridges and } \\
\text { mesas, with 2-percent } \\
\text { slopes separated by } \\
\text { broad valleys having } \\
\text { low relief and numer- } \\
\text { ous drainages and } \\
\text { ephemeral streams; } \\
\text { sandstone escarp- } \\
\text { ments adjacent to } \\
\text { mined lands. Older } \\
\text { unreclaimed spoils } \\
\text { have } 35 \text {-percent } \\
\text { slopes and some } \\
\text { minor rilling and } \\
\text { gullying. Some wind } \\
\text { erosion. } \\
3,260-3,340\end{array}$ \\
\hline
\end{tabular}

\footnotetext{
'See Gikawn (1979) for nublisbed reclamation data on specific mines.
} 


\begin{tabular}{cc} 
Growing Media & Vegetation \\
\hline Premining: No data. & Premining: Mostly grasses (wheatgrass-needlegrass). \\
Postmining: In older recla- & Postmining: Successful seeding of native grasses and \\
mation, fine-textured & grain crops achieved on shallow, nontoxic spoils \\
spoils with high sodium & or on topsoiled surfaces. Little success in \\
content usually mixed in & rehabilitating raw spoils having high sodium con- \\
handling; some highly & tent. Western and crested wheatgrasses, green \\
sodic spoils still exposed; & needlegrass, slender wheatgrass, yellow \\
topsoil not used. Recent & sweetclover, and Sudan grass cover. Planted and \\
reclamation, including & self-sown trees and shrubs on old spoil piles.
\end{tabular}
reworking of older areas, has involved up to 21 in. of topsoil.
Species Diversity

Premining: Mostly mixed grasses.

\section{Postmining: Mostly} grasses. Experimental plantings of exotic trees and shrubs on rougher ground. Cottonwood, willow, and common reed volunteers in wetter areas.
Premining: Clay loam to cobbly loam soils, 10 to $40 \mathrm{in}$. deep, moderate permeability; pH 7.2-8.0.

Postmining: Smoothed surface, generally 6 to 12 in. topsoil, but as much as 36 in. on highly sodic spoils; fertilized.
Premining: Ponderosa pine forests on ridges and buttes, shrub-grassland on slopes, and wheatgrass-needlegrass in bottom lands.

Postmining: Vegetation cover variable ( 4 to 22 percent). Slender wheatgrass and associated wheatgrasses dominant. Lesser amounts of yellow sweetclover and winter wheat. Preliminary results from ponderosa pine revegetation show 80 -percent survival of transplanted containerized trees and 33-percent survival of transplanted bare-rooted trees.
Premining: About 20 species.

Postmining: Mostly grasses; diversity increased by transplanting local ponderosa pines and associated vegetation. Eight species of shrubs and two species of deciduous trees planted.
Premining: No data.

Postmining: 320-900 lbs/acre for ponderosa pine area; 3,000 $\mathrm{lbs} /$ acre for shrub grass area.
Premining: Sandy loam over
parent sandstone and shale; $\mathrm{pH} 7.0-8.2$; toxic materials absent in surface zones.

Postmining: Selective salvage and redistribution of topsoil and subsoil; stockpiling avoided. Straw mulch incorporated.
Premining: Mixed prairie (wheatgrasses, needlegrass, grama, sedge, silver sagebrush, big sagebrush, fringed sagebrush, broom snakeweed) with scattered woodland of ponderosa pine and juniper; intrusions of niparian plants (cottonwood, boxelder, chokecherry, snowberry).

Postmining: 40-percent cover and litter accumulation in basins; cover of needleandthread; bluebunch wheatgrass; threadleaf sedge; tall, crested, western, slender, and thickspike wheatgrasses; blue grama; prairie sandreed, cheatgrass; bluegrass; and bluestem. Upland areas covered by tall and crested wheatgrasses, Indian ricegrass, and others; cover 25 percent. Research plots monitored by Montana State University. Early reclamation by broadcast, drill, and aerial seeding with crested wheatgrass, thickspike wheatgrass, western wheatgrass, smooth brome, orchardgrass, alfalfa, sainfoin, fourwing saltbush, and similar plants.

Premining: Over 200
species on rangeland
sites.
Postmining: 1972 seed
mix contained 16
species. After 1973 ,
refined seed mix of
14 major perennial
species was used.

Premining: 250-800 lbs/acre; $500-1,600 \mathrm{lbs} /$ acre potential for wetter areas.

Postmining: 3,000 lbs/acre for introduced stands. 


\begin{tabular}{|c|c|c|c|c|c|}
\hline Vline and locasion & $\begin{array}{l}\text { Mining } \\
\text { Begunning date } \\
\text { Acres muned }\end{array}$ & $\begin{array}{l}\text { Reclamation } \\
\text { Begnnung date } \\
\text { Acres reclaumed }\end{array}$ & Land Use & Climalic Factors & $\begin{array}{c}\text { Topography } \\
\text { Elevation, in feet }\end{array}$ \\
\hline $\begin{array}{l}\text { 4. BIG SKY MINE } \\
\text { Colstrip. Rosebud Coun- } \\
\text { ty, southeastern } \\
\text { Montana } \\
\text { USGS } 7 y^{\prime} \text { ' maps: } \\
\text { Colstrip SE. } \\
\text { lat } 45^{\circ} 49^{\prime} \mathrm{N} \text {.; } \\
\text { long } 106^{\circ} 36^{\prime} \mathrm{W} \text {. } \\
\text { secs. } 21,22,27, \mathrm{~T} .1 \mathrm{~N} . \\
\text { R. } 41 \mathrm{E} \text {. }\end{array}$ & $\begin{array}{l}1969 \\
674 \text { acres } \\
\text { through } \\
1978 .\end{array}$ & $\begin{array}{l}521 \text { acres graded, } \\
346 \text { acres seeded } \\
\text { through } 1978 .\end{array}$ & $\begin{array}{l}\text { Premining: } \\
\text { Wildlife and } \\
\text { livestock } \\
\text { range. } \\
\text { Intended: No } \\
\text { change. } \\
\text { Livestock and } \\
\text { hunting ex- } \\
\text { cluded dur- } \\
\text { ing reclama- } \\
\text { tion; live- } \\
\text { stock grazing } \\
\text { scheduled to } \\
\text { begin on } 335 \\
\text { acres in } \\
\text { 1980. }\end{array}$ & $\begin{array}{l}\text { Growing season } \\
\text { (avg frost-free days): } 127 \\
\text { mid-May to mid- } \\
\text { September. } \\
\text { Temperature }\left({ }^{\circ} \mathrm{F}\right) \text { : } \\
\quad \text { mean annual: } 47 \\
\text { range: }-40 \text { to } 110 \text {. } \\
\text { Precipitation (inches): } \\
\text { mean annual: } 15 \\
\text { range: } 14 \text { to } 16 ; \\
60 \text { percent from March } \\
\text { through June. }\end{array}$ & $\begin{array}{l}\text { Rolling hills, sandstone } \\
\text { mesas with } 6-\text { to } \\
\text { 8-percent slopes; } \\
\text { highwalls reduced to } \\
20 \text { percent. Local } \\
\text { gullying repaired with } \\
\text { straw mulch; wind } \\
\text { erosion on temporari- } \\
\text { ly bare flat areas. } \\
3,220-3,300\end{array}$ \\
\hline $\begin{array}{l}\text { 5. WEST DECKER MINE } \\
\text { Decker, Big Horn Coun- } \\
\text { ty, southeastern } \\
\text { Montana } \\
\text { USGS } 71^{\prime} \text { ' }^{\prime} \text { maps: } \\
\text { Decker } \\
\text { lat } 45^{\circ} 03^{\prime} \mathrm{N} \text {; } \\
\text { long } 106^{\circ} 51^{\prime} \mathrm{W} \text {. } \\
\text { secs. } 8,9,16,17, \mathrm{~T} .9 \mathrm{~S} . \\
\text { R. } 40 \mathrm{E} \text {. }\end{array}$ & $\begin{array}{l}1972 \\
636 \text { acres } \\
\text { through } \\
1978 .\end{array}$ & $\begin{array}{l}1975 \\
216 \text { acres through } \\
1978 .\end{array}$ & $\begin{array}{l}\text { Premining: } \\
\text { Wildlife and } \\
\text { livestock } \\
\text { range; some } \\
\text { wheat. } \\
\text { Intended: No } \\
\text { change. } \\
\text { Livestock and } \\
\text { hunting ex- } \\
\text { cluded dur- } \\
\text { ing reclama- } \\
\text { tion. }\end{array}$ & $\begin{array}{l}\text { Growing season } \\
\text { (avg frost-free days): } 107 \\
\text { June to mid-September. } \\
\text { Temperature ( }{ }^{\circ} \mathrm{F} \text { ): } \\
\text { mean annual: } 46 \\
\text { range: }-43 \text { to } 108 \text {. } \\
\text { Precipitation (inches): } \\
\text { mean annual: } 14 \\
\text { range: No data; } \\
50 \text { percent from April } \\
\text { through June. }\end{array}$ & $\begin{array}{l}\text { Rolling hills with } 2-\text { to } \\
10 \text {-percent slopes and } \\
\text { divides and broad } \\
\text { drainage bottoms. } \\
\text { Outcrops of red } \\
\text { clinker and shale. } \\
\text { Some minor rilling. } \\
3,500-3,600\end{array}$ \\
\hline $\begin{array}{l}\text { 6. BELLE AYR } \\
\text { SOUTH MINE } \\
\text { Gillette, Cambell } \\
\text { County, nor- } \\
\text { theastern Wyoming } \\
\text { USGS } 71^{\prime} 2^{\prime} \text { maps: } \\
\text { Saddle Horse Butte } \\
\text { lat } 44^{\circ} 06^{\prime} \mathrm{N} \text {.; } \\
\text { long } 105^{\circ} 22^{\prime} \mathrm{W} \text {. } \\
\text { secs. } 27,28,33,34, \\
\text { T. } 48 \mathrm{~N}, \text { R., R. W. }\end{array}$ & $\begin{array}{c}1,122 \text { acres } \\
\text { through } \\
1978 .\end{array}$ & $\begin{array}{l}424 \text { acres of com- } \\
\text { pleted } \\
\text { reclamation } \\
\text { through } 1978 .\end{array}$ & $\begin{array}{l}\text { Premining: } \\
\text { Livestock } \\
\text { range. } \\
\text { Intended: No } \\
\text { change. } \\
\text { Livestock and } \\
\text { hunting ex- } \\
\text { cluded dur- } \\
\text { ing reclama- } \\
\text { tion. }\end{array}$ & $\begin{array}{l}\text { Growing season } \\
\text { (avg frost-free days): } 114 \text {. } \\
\text { Temperaiure ( }{ }^{\circ} \mathrm{F} \text { : } \\
\text { mean annual: } 45 \\
\text { range: }-32 \text { to } 100 \text {. } \\
\text { Precipitation (inches): } \\
\text { mean annual: } 15 \\
\text { range: No data; } \\
50 \text { percent from April } \\
\text { through June. }\end{array}$ & $\begin{array}{l}\text { Rolling hills with } 4 \text { - to } \\
8 \text {-percent slopes; nar- } \\
\text { row valleys. Spoils } \\
\text { reshaped to nearly } \\
\text { original configuration } \\
\text { with minor rilling of } \\
\text { mined surfaces; heavy } \\
\text { sheetwash during } \\
\text { seasonal storms. } \\
4,460-4,520\end{array}$ \\
\hline
\end{tabular}

\footnotetext{
'See Gikawn (I979) for published reclamation data on snecific mmes.
} 
Premining: Silty to sandy Premining: Mostly prairie grasslands merging with Premining: Over 21 soil, shallow to moderately deep over sandstone and shale; $\mathrm{pH}$ 7.1-8.3; blue-gray partings in coal appear to be toxic to plants.

Postmining: High sodium content in pre-1973 mixed spoils; after 1973. spoils topsoiled, and partings buried minimum of $8 \mathrm{ft}$. ponderosa pine; scattered mixed shrub and grass on upland slopes and mesas. Silver sagebrush, needleandthread, wheatgrasses, and blue grama Postmining: Mostly dominant.

Postmining: Thickspike, western, and crested wheatgrasses; smooth brome; alfalfa; and sweetclover dominant; some native and exotic woody plants. Cover ranges from 5 to 40 percent. Russian-thistle dominant in some areas. Some litter development on 1974 and 1975 spoils. Some large transplanted junipers and pines have sur. vived; ponderosa pine beginning to seed-in on adjacent reclaimed slopes. species

limited to a few grasses and nonnative legumes. A few native trees being transplanted. Seedings no longer contain nonnative species.
Premining: 420 to $968 \mathrm{lbs} /$ acre; 900 to $\mathrm{l}, 400 \mathrm{lbs} /$ acre potential.

Postmining: Prior to 1973,248 Ibs/acre. Topsoiled spoils greater than $2,200 \mathrm{lbs} /$ acre (for northeast exposure). First mowing of western wheatgrass, smooth brome, and yellow sweetclover yield ed 3,000 lbs/acre.
Premining: Sandy loam to Premining: Mainly mid-shortgrass prairie; prairie Premining: About 18 clay, moderately deep to deep over parent shale and sandstone; average soil depth about 20 in.; high sodium content in clay material; $\mathbf{p H}$ 6.6-8.9.

Postmining: Some areas topsoiled with sandy loam to depth of 18 to 20 in.; fertilized, mulched. High sodium content of some spoils deters plant growth in nontopsoiled areas. sandreed, little bluestem, blue grama, needleandthread, Sandberg bluegrass, and junegrass; cover 20 to 30 percent. Riparian vegetation includes western wheatgrass, Japanese brome, green needlegrass, big sagebrush, and silver sagebrush. Some riparian areas contain black greasewood, blue grama, bluegrass, bluestem; cover averages 50 percent.

Postmining: Slender, western, and thickspike wheatgrasses, and smooth brome. Ground cover ranges from uniform ( 37 percent) to variable and is as much as 95 percent in grassland-sagebrush zone. In areas of fourwing saltbush and sagebrush, cover of volunteer seedlings averages 65 percent.
Premining: 1, $100 \mathrm{lbs} /$ acre averspecies; mostly grasses and forbs, both annual and perennial.

Postmining: 11 species in seed mix; 3 species hand planted; 13 volunteer species. age, up to $2,000 \mathrm{lbs} /$ acre in good years; highly variable with aspect (exposure).

Postmining: 1,834 lbs/acre average yield after 2 years. Ranges from 500 to 5,230 lbs/acre.
Premining: Deep to moder- Premining: Grassland-sagebrush; big sagebrush, Premining: About 12 ately deep sandy clay loams to shallow loams; locally rocky; $\mathrm{pH}$ $5.3-7.9$.

Postmining: Ripped and covered with $2 \mathrm{ft}$ of sandy loam topsoil; fertilized, mulched. No toxic soil problems. High sodium content in some spoils. western wheatgrass, junegrass, and Sandberg species.

bluegrass dominant. Some riparian vegetation.

Postmining: Alfalfa dominant on older (1974) topsoil stockpiles which have been seeded with tall wheatgrass, smooth brome, alfalfa, yellow sweetclover, and crested wheatgrass; summer-cypress is also abundant. Cover ranges from 0 to 40 percent, averaging 15 percent. On more recently reclaimed areas, summer-cypress dominates, providing up to 75 percent cover. Several tree species planted experimentally and to provide cover, usually associated with reclaimed stream channels or artificial rock piles. Winter wheat used as nurse crop. In 1973, small spoil area had fourwing saltbush, wheatgrasses, and green needlegrass.
Premining: 185-625 lbs/acre (overgrazed); $1,000-2,000$ Ibs/acre potential.

Postmining: Variable, but limited; test plots Postmining: 170-566 lbs/acre on under study. Several seed mixes used. A representative seed mix contains 20 species. 1-year-old seeded areas; 820 lbs/acre yield for a small test area seeded in 1973 without topsoil. 


\begin{tabular}{|c|c|c|c|c|c|}
\hline Mine and location & $\begin{array}{c}\text { Minung } \\
\text { Bcenning date } \\
\text { Acres muned }\end{array}$ & $\begin{array}{l}\text { Reclamation } \\
\text { Bcginning date } \\
\text { Acres reclaumed }\end{array}$ & Land Use & Climatic Factors & $\begin{array}{c}\text { Topography } \\
\text { Elevation, in feet }\end{array}$ \\
\hline $\begin{array}{l}\text { 7. DAVE JOHNSTON } \\
\text { MIINE } \\
\text { Glenrock, Converse } \\
\text { County, east-central } \\
\text { Wyoming } \\
\text { USGS } 15^{\prime} \text { maps: } \\
\text { Fifty-Five Ranch } \\
\text { lat } 43^{\circ} 05^{\prime} \mathrm{N} \text {.; } \\
\text { long } 105^{\circ} 50^{\prime} \mathrm{W} \text {. } \\
\text { secs. } 2,3,10,15,21,28,34, \\
\text { T. } 36 \mathrm{~N}, \mathrm{R}^{2} 75 \mathrm{~W} \text {. }\end{array}$ & $\begin{array}{l}\text { Surface: } 1958 . \\
\text { 2,206 acres } \\
\text { through } \\
\text { March } 1979 .\end{array}$ & $\begin{array}{l}1965 \\
1,187 \text { acres } \\
\text { through } \\
\text { March } 1979 .\end{array}$ & $\begin{array}{l}\text { Premining: } \\
\text { Wildlife and } \\
\text { livestock } \\
\text { range. } \\
\text { Intended: No } \\
\text { change. } \\
\text { Livestock and } \\
\text { hunting ex- } \\
\text { cluded dur- } \\
\text { ing reclama- } \\
\text { tion. }\end{array}$ & $\begin{array}{l}\text { Growing season } \\
\text { (avg frost-free days): } 125 \text {. } \\
\text { Temperature ( }{ }^{\circ} \mathrm{F} \text { ): } \\
\text { mean annual: } 45 \\
\text { range: }-35 \text { to } 100 . \\
\text { Precipitation (inches): } \\
\text { mean annual: } 13 \\
\text { range: } 12 \text { to } 14 ; \\
60 \text { percent from April } \\
\text { through October. }\end{array}$ & $\begin{array}{l}\text { Rolling hills with 4-to } \\
6 \text {-percent slopes. } \\
\text { Spoils regraded to } \\
\text { nearly original con- } \\
\text { figuration. Local } \\
\text { gullying of leveled } \\
\text { spoils and cuts. Wind } \\
\text { erosion of sandy } \\
\text { ridgetops and soil- } \\
\text { stripped surfaces. } \\
5,800-6,200\end{array}$ \\
\hline $\begin{array}{l}\text { 8. ROSEBUD, MEDICINE } \\
\text { BOW, SEMINOE I } \\
\text { and } 2 \text { MINES } \\
\text { Hanna, Carbon County, } \\
\text { south-central } \\
\text { Wyoming } \\
\text { USGS } 71^{\prime}{ }^{\prime} \text { maps: } \\
\text { Tenmile Spring } \\
\text { Elmo } \\
\text { Como } \\
\text { Dana } \\
\text { Hanna } \\
\text { lat } 41^{\circ} 55^{\prime} \mathrm{N} . ; \\
\text { long } 106^{\circ} 33^{\prime} \mathrm{W} . \\
\text { T.22-23 N., } \\
\text { R. } 81-82 \mathrm{~W} .\end{array}$ & $\begin{array}{l}\text { Rosebud: } 1920 \\
\text { Seminoe } \\
\text { No. I: } 1970 . \\
\text { Seminoe } \\
\text { No. 2: } 1973 \text {. } \\
\text { Rosebud: } 3,743 \\
\text { acres } \\
\text { through } \\
\text { 1978. } \\
\text { Medicine Bow: } \\
\text { 1,319 acres } \\
\text { through } \\
\text { 1978. } \\
\text { Seminoe No. I: } \\
\text { 1,500 acres } \\
\text { through } \\
\text { 1970-76. } \\
\text { Seminoe No. } 2: \\
826 \text { acres } \\
\text { through } \\
1978 \text {. }\end{array}$ & $\begin{array}{l}\text { Unknown. } \\
\text { Rosebud: } 661 \\
\text { acres. } \\
\text { Medicine Bow: } \\
304 \text { acres. } \\
\text { Seminoe No. 1: } \\
600 \text { acres } \\
\text { Seminoe No. 2: } \\
369 \text { acres. }\end{array}$ & $\begin{array}{l}\text { Premining: } \\
\text { Wildlife and } \\
\text { livestock } \\
\text { range. } \\
\text { Intended: No } \\
\text { change. }\end{array}$ & $\begin{array}{l}\text { Growing season } \\
\text { (avg frost-free days): } 86 \\
\text { June } 7 \text { to August } 30 \text {. } \\
\text { Temperature }\left({ }^{\circ} \mathrm{F}\right) \text { : } \\
\text { mean annual: } 42 \\
\text { range: }-36 \text { to } 98 \text {. } \\
\text { Precipitation (inches): } \\
\text { mean annual: } 10 \\
\text { range: No data. }\end{array}$ & $\begin{array}{l}\text { Cuestas, mesas, rolling } \\
\text { hills with } 6-\text { to } 8 \text {-per- } \\
\text { cent slopes separated } \\
\text { by valleys. Spoils } \\
\text { graded to long, } \\
\text { straight slopes (both } \\
\text { vertically and along } \\
\text { the slope) approxi- } \\
\text { mating the dip of the } \\
\text { original strata. Old } \\
\text { pits of Rosebud mine } \\
\text { contain water; maxi- } \\
\text { mum slopes } 25 \text { per- } \\
\text { cent. Gullying and } \\
\text { severe rilling on some } \\
\text { steep spoils. } \\
6,800-7,200\end{array}$ \\
\hline $\begin{array}{l}\text { 9. ELKOL-SORENSEN } \\
\text { MINE } \\
\text { Kemmerer, Lincoln } \\
\text { County, southcastern } \\
\text { Wyoming } \\
\text { USGS } 71^{\prime} /^{\prime} \text { maps: } \\
\text { Elkol } \\
\text { USGS } 15^{\prime} \text { maps: } \\
\text { Kemmerer } \\
\text { lat } 41^{\circ} 45^{\prime} \mathrm{N} . \text {; } \\
\text { long } 110^{\circ} 37^{\prime} \mathrm{W} \text {. } \\
\text { sec. } 3, \mathrm{~T} .20 \mathrm{~N} . \\
\text { R. } 116 \mathrm{~W} \text {. } \\
\text { sec. } 32, \\
\text { T. } 21 \mathrm{~N} ., \mathrm{R} .116 \mathrm{~W} \text {. }\end{array}$ & $\begin{array}{l}\text { Underground: } \\
1909-50 . \\
\text { Surface: } 1950 . \\
440 \text { acres mined } \\
\text { through } \\
1978 .\end{array}$ & $\begin{array}{l}1973 \\
\text { Numerous test } \\
\text { areas } 1965-69 . \\
752 \text { acres shaped, } \\
304 \text { acres } \\
\text { completed. }\end{array}$ & $\begin{array}{l}\text { Premining: } \\
\text { Wildlife and } \\
\text { livestock } \\
\text { range. } \\
\text { Intended: No } \\
\text { change. } \\
\text { Livestock ex- } \\
\text { cluded dur- } \\
\text { ing reclama- } \\
\text { tion. }\end{array}$ & $\begin{array}{l}\text { Growing season } \\
\text { (avg frost-free days): } 60 \\
\text { mid-June to mid- } \\
\text { August. } \\
\text { Temperature }\left({ }^{\circ} \mathrm{F}\right) \text { : } \\
\text { mean annual: } 40 \\
\text { range: }-32 \text { to } 98 \text {. } \\
\text { Precipitation (inches): } \\
\text { mean annual: } 9 \\
\text { range: No data. } \\
50 \text { percent from April } \\
\text { through August. All } \\
\text { seeded areas irrigated } \\
\text { for } 2 \text { years with two } \\
\text { applications per year by } \\
\text { sprinkler system. }\end{array}$ & $\begin{array}{l}\text { Cuestas with } 25-\text { to } \\
40 \text {-percent slopes } \\
\text { separated by broad } \\
\text { valleys. Regional dip } \\
\text { is westward. Spoils } \\
\text { graded to long, undu- } \\
\text { lating configuration } \\
\text { approximating origi- } \\
\text { nal dip. Water in } \\
\text { final boxcuts to depth } \\
\text { of } 50 \mathrm{ft} \text {. Steep slopes } \\
\text { are rilled and gullied. } \\
7,000-7,300\end{array}$ \\
\hline
\end{tabular}


Premining: Deep to moder- Premining: Grassland-sagebrush; big sagebrush, rab- Premining: Over 15 ately deep sandy to clayey loams over mostly sandstone and interbedded thin shale; permeability moderate to rapid; sodium locally high in some soils; $\mathrm{pH}$ 3.6-7.0.

Postmining: Graded spoil covered by 6 to 18 in. mixed topsoil; pitted, mulched, and fertilized. bitbrush, blue grama, junegrass, and needleandthread dominant. Cover varies from 0 to 40 percent.

Postmining: Revegetated areas vary considerably with age and location; ground cover varies from 0 to 35 percent. Dominant species include western, streambank, thickspike, and crested wheatgrasses. Millet used as a cover crop. Initially, seeding was done without topsoil or mulch; created wheatgrass, alfalfa, and cereal rye were used. Wide variety of grasses now used with topsoil and mulch. species.

Postmining: 7 species in seed mix.
Premining: Probably low.

Postmining: 1972 seeding in untopsoiled areas yielded 73 lbs/acre. Topsoiled areas yielded $1,300 \mathrm{lbs} /$ acre.
Premining: Mostly brown sandy loam over lime- stone and sandstone; also, friable grayish- brown loam over shales with free lime usually present. $\mathrm{pH} 7.0-9.0$;
Postmining: Spoils com- Postmining: Drill seeding of wheatgrasses and yellow posed of shale, sand- stone, and clay; most areas topsoiled. Straw and sprayed fibrous mulch. brush, and saltbush with Indian ricegrass, needleandthread, and junegrass. Local saline soils, characterized by poor drainage and heavy clay soils, are distinguished by the presence of greasewood, saltbush, and saltgrass. Cover of 24 to 76 percent.
sweetclover, Bluebunch wheatgrass, green needlegrass, Indian ricegrass, streambank wheatgrass, western wheatgrass, thickspike wheatgrass, slender wheatgrass, fourwing saltbush, and shadscale saltbush. Cover uniform but varies with age of seeding from 79 to 89 per- cent. Some sagebrush, saltbush, and greasewood volunteers.

Premining: Big sagebrush, black sagebrush, rabbit- Premining: Over 22 species.

Postmining: 45 species; ricegrasses on steep spoils.
Premining: 600 to $1,400 \mathrm{lbs} / \mathrm{acre}$ on big sage-grassland; 250-600 lbs/acre on low sage-saltbush type. Seminoe No. $2-740$ to 1,000 lbs/acre.

Postmining:

Rosebud-78 to $412 \mathrm{lbs} / \mathrm{acre}$ (1-year growth).

Medicine Bow-No data.

Seminoe No. 1-538 to 888 lbs/acre; Russian-thistle severely invaded seeded areas causing competition for available moisture during first 2 years, then thinned out.

Seminoe No. 2-579 to 1,728 lbs/acre (1-year growth).
Premining: Thin, rocky, clay loam over parent shale and sandstone; $\mathrm{pH}$ 6.5-7.4; no toxic materials.

Postmining: Surface materia of shale, clay, and weathered shale; 6 to 8 in. of topsoil used locally. Older spoils weathered and rounded. Tops furrowed.

\footnotetext{
Premining: Sagebrush, shadscale, rabbitbrush, greasewood, antelope bitterbrush, western wheatgrass, junegrass, slender wheatgrass, Indian serviceberry, rose, and Great Basin wildrye.
} ricegrass; some areas contain aspen, chokecherry, Postmining: Limited diversity.

Premining: Over 15 No data.
species.
Postmining: Limited
diversity.




\begin{tabular}{|c|c|c|c|c|c|}
\hline Mine and location & $\begin{array}{l}\text { Munung } \\
\text { Beginning date } \\
\text { Acres mined }\end{array}$ & $\begin{array}{l}\text { Reclamation } \\
\text { Bkginning date } \\
\text { Acres reclaimed }\end{array}$ & Land Use & Climatic Factors & $\begin{array}{c}\text { Topography } \\
\text { Elevation, in feet }\end{array}$ \\
\hline $\begin{array}{l}\text { 10. ENERGY 1, 2, and } 3 \\
\text { MIINES } \\
\text { Oak Creek, Routt } \\
\text { County, north- } \\
\text { western Colorado } \\
\text { USGS } 71^{\prime}{ }^{\prime} \text { maps: } \\
\text { Rattlesnake Butte } \\
\text { lat } 40^{\circ} 20^{\prime} \mathrm{N} \text {.; } \\
\text { long } 107^{\circ} 05^{\prime} \mathrm{W} \text {. } \\
\text { T. } 4-5 \mathrm{~N} ., \mathrm{R} .86-87 \mathrm{~W} \text {. }\end{array}$ & $\begin{array}{l}\text { Energy No. 1: } \\
\quad 1962 . \\
\text { Energy No. 2: } \\
\quad 1972 . \\
\text { Energy No. 3: } \\
\quad 1975 .\end{array}$ & $\begin{array}{l}\text { Energy No. 1: } \\
\text { 1965-68- } \\
\text { spoil tops lev- } \\
\text { eled and } \\
\text { aerially } \\
\text { seeded; } 292 \\
\text { acres. } \\
\text { 1976- } \\
\text { grading, top- } \\
\text { soiling, and } \\
\text { seeding. } \\
\text { Energy No. 2: } \\
\text { 1974- } \\
\text { grading, top- } \\
\text { soiling and } \\
\text { seeding. } \\
\text { Energy No. 3: } \\
\text { 1975- } \\
\text { grading, top- } \\
\text { soiling, and } \\
\text { seeding. }\end{array}$ & $\begin{array}{l}\text { Premining: } \\
\text { Wildlife and } \\
\text { livestock } \\
\text { range, wheat } \\
\text { farming, and } \\
\text { dry pasture. } \\
\text { Current: } \\
\text { Cropland, } \\
\text { hay } \\
\text { meadows, } \\
\text { livestock } \\
\text { range, and } \\
\text { wildlife } \\
\text { feeding } \\
\text { areas. } \\
\text { Intended: No } \\
\text { change. }\end{array}$ & $\begin{array}{l}\text { Growing season } \\
\text { (avg frost-free days): } 65 \\
\text { mid-June to September. } \\
\text { Temperature }\left({ }^{\circ} \mathrm{F}\right) \text { : } \\
\text { mean annual: } 42 \\
\text { range: } 26 \text { to } 58 \text {. } \\
\text { Precipitation (inches): } \\
\text { mean annual: } 16 \\
\text { range: No data. } \\
50 \text { percent as snow. }\end{array}$ & $\begin{array}{l}\text { Hills and sandstone } \\
\text { cuestas. Spoils gently } \\
\text { dipping and undulat- } \\
\text { ing. Old spoil tops } \\
\text { leveled and separated } \\
\text { by V-shaped valleys, } \\
13 \text { to } 20 \mathrm{ft} \text { deep. } \\
\text { Spoils reclaimed after } \\
1974 \text { reshaped to near } \\
\text { original configuration. } \\
\text { Energy No. } 2 \text { final } \\
\text { boxcut backfilled and } \\
\text { leveled to undulating, } \\
\text { hummocky, dipping } \\
\text { surface approximating } \\
\text { original topography. } \\
6,900-7,600\end{array}$ \\
\hline $\begin{array}{l}\text { 11. SENECA } 1 \text { and } \\
2 \text { MINES } \\
\text { Hayden, Routt County, } \\
\text { northwestern } \\
\text { Colorado } \\
\text { USGS } 71^{\prime} \text { maps: } \\
\text { Mount Harris } \\
\text { Milner } \\
\text { lat } 40^{\circ} 27^{\prime} \mathrm{N} \text {; } \\
\text { long } 107^{\circ} 07^{\prime} \mathrm{W} \text {. } \\
\text { T.5-6 N., R.87 W. }\end{array}$ & $\begin{array}{l}\text { Unknown. } \\
\text { Underground: } \\
\text { Grassy and } \\
\text { Wolf } \\
\text { Creek } \\
\text { mines. } \\
\text { Surface: } \\
\text { Seneca No. } \\
\text { I-119 acres } \\
\text { through } \\
1964-69 . \\
\text { Seneca No. } \\
2-600 \text { acres } \\
\text { through } \\
1968-76 .\end{array}$ & $\begin{array}{l}\text { Seneca No. 1: } \\
1969 \\
\text { Early spoils } \\
\text { reshaped to } \\
\text { original con- } \\
\text { tours; lower } \\
\text { spoil slopes } \\
\text { have flattened } \\
\text { ridges. }\end{array}$ & $\begin{array}{l}\text { Premining: } \\
\text { Wildlife and } \\
\text { livestock } \\
\text { range. } \\
\text { Intended: No } \\
\text { change. }\end{array}$ & $\begin{array}{l}\text { Growing season } \\
\text { (avg frost-free days): } 81 \\
\text { mid-June to early } \\
\text { September. } \\
\text { Temperature ( }{ }^{\circ} \mathrm{F} \text { ): } \\
\text { mean annual: } 41 \\
\text { range: }-42 \text { to } 100 \text {. } \\
\text { Precipitation (inches): } \\
\text { mean annual: } 16 \\
\text { range: No data. } \\
50 \text { percent as snow; } \\
1 \text { in./month during the } \\
\text { growing season. }\end{array}$ & $\begin{array}{l}\text { Hilly, with } 17 \text { - to } 20 \text { - per- } \\
\text { cent slopes. Seneca } \\
\text { No. I-steep, exten- } \\
\text { sive spoil areas having } \\
\text { different slopes and } \\
\text { aspect; ridgetops flat- } \\
\text { tened to } 15 \text { ft. Seneca } \\
\text { No. } 2 \text {-large, exten- } \\
\text { sive areas graded to } \\
\text { near original con- } \\
\text { figuration. Local } \\
\text { slumping of spoil sta- } \\
\text { bilized by horizontal } \\
\text { trenches and berms; } \\
\text { wind ablation on high } \\
\text { ridges. Some steep } \\
\text { slopes gullied. } \\
6,640-7,600\end{array}$ \\
\hline $\begin{array}{l}\text { 12. WEBER AND } \\
\text { CHAPPEL MINES } \\
\text { Coalville, Summit } \\
\text { County, north-central } \\
\text { Utah } \\
\text { USG.S } 7 y_{2}^{\prime} \text { maps: } \\
\text { Turner Hollow } \\
\text { lat } 40^{\circ} 56^{\prime} \text { N.; } \\
\text { long } 111^{\circ} 22^{\prime} \mathrm{W} . \\
\text { secs. } 2 \text { and } \\
3, \text { T. } 2 \text { N., R.5 L: }\end{array}$ & $\begin{array}{l}\text { Beginning dates } \\
\text { unknown. } \\
\text { Underground: } \\
\text { Weber } \\
\text { (closed in } \\
1950 \text { 's). } \\
\text { Chappell } \\
\text { (closed in } \\
1960 \text { 's). } \\
\text { Surface: } \\
\text { Weber: } 20 \\
\text { acres. }\end{array}$ & $\begin{array}{l}\text { No reclamation; } \\
\text { abandoned in } \\
\text { early } 1960 \text { 's. } \\
\text { Some leveling } \\
\text { for oil well } \\
\text { drilling. }\end{array}$ & $\begin{array}{l}\text { Premining: } \\
\text { Wildlife and } \\
\text { livestock } \\
\text { range. } \\
\text { Current: No } \\
\text { change. }\end{array}$ & $\begin{array}{l}\text { Growing season } \\
\text { (avg frost-free days): } 70 \\
\text { (estimate). } \\
\text { Temperature }\left({ }^{\circ} \mathrm{F} \text { ): }\right. \\
\text { mean annual: } 44 \\
\text { (estimate) } \\
\text { range: No data. } \\
\text { Precipitation (inches): } \\
\text { mean annual: } 14 \\
\text { festimate) } \\
\text { range: No data. }\end{array}$ & $\begin{array}{l}\text { Mountain valleys, aban- } \\
\text { doned coal dumps. } \\
\text { Slope: Weber, } 20 \text { per- } \\
\text { cent; Chappell, } 50 \\
\text { percent. Coal fines } \\
\text { erosion. } \\
\text { Weber, } 5,900 \text {; } \\
\text { Chappell, } 6,200\end{array}$ \\
\hline
\end{tabular}


Premining: Silty clay, silty Premining: Energy No. I-sagebrush, mountain Premining: Over 20 clay loams, clay loams, loams, and fine sandy loams over sandstone and shale parent material; pH 5.9-8.3.

Postmining: All leveled spoils covered with topsoil or subsoil; no topsoiling done on shales or sandstones; older spoils topsoiled at mines with considerable amount of subsoil. Contour furrows and berms constructed to control erosion. mahogany-scrub oak, and aspen. Primary plants are oak, serviceberry, chokecherry, snowberry, smooth brome, mountain brome, and forbs. Energy No. 2-sagebrush ground layer dominated by western wheatgrass and weedy annuals. Local stands of dense aspen and cultivated wheat fields.

Postmining: Ground cover on sites 3 years old exceeds 35 percent; on sites 5 years old, exceeds 50 percent. Clumps of aspen, serviceberry, and oak set out on smoothed spoil before topsoiling. Rockpiles added for habitat. Energy No. 1-wheat and rye nurse crop was highly successful. Energy No. 2-surviving species are intermediate wheatgrass, smooth brome, and alfalfa. Annual grasses and forbs now crowded out by planted perennial species.
Premining: species.

Postmining: Range of 7 to 34 species in 1979. 1977 and 1978 planting mix of 24 species; 1979 planting mix of 27 species.

Wide variety of grasses, forbs, and shrubs.
Energy No. 1-507 Jbs/acre for aspen type to $2,500 \mathrm{lbs} /$ acre for tall sage-grass type (excluding aspen and shrubs over $4.5 \mathrm{ft}$ ).

Energy No. 2-917 lbs/acre.

Energy No. 3-347 lbs/acre.

Postmining:

Energy No. $1-280$ to 2,467 lbs/acre for nontopsoiled, seeded spoils; 1,260-1,900 lbs/acre for topsoiled, seeded spoils.

Energy No. 2-3,000 lbs/acre after 4 years.

Energy No. 3-826 lbs/acre in $1978 ; 1,700 \mathrm{lbs} /$ acre in 1979.
Premining: Deep loams, clay loams, silt loams, and minor clay pans over sandstone and shale; $\mathrm{pH}$ 6.6-7.9.

Postmining: Seneca No. I-spoil tops flattened but not topsoiled. Seneca No. 2-spoils of clay, shale, sandstone; some locations not topsoiled. Straw mulch.
Premining: Mixture of grassland-shrub; big Premining: About 18 sagebrush, big bluegrass, needleandthread, snowberry, Gambel oak, serviceberry. Cover 40 to 70 percent.

Postmining: Seneca No. 1-revegetated with airseeded alfalfa; smooth brome; crested, intermediate, and pubescent wheatgrasses. Orchardgrass, cheatgrass, and brome dominant. Survival of transplanted, mostly introduced deciduous shrubs and trees good. Vegetation sparse on some slopes, rocky areas, and coal fines. Sagebrush volunteers common in some areas. Seneca No. 2-no areas successfully revegetated by native species through 1976 , but some alfalfa cover. Revegetated areas dominated by yellow sweetclover, smooth brome, crested wheatgrass, and intermediate wheatgrass. Cover ranges from 27 to 34 percent. species.

Postmining: About 13 species of grasses and legumes, most not native.
Premining: Seneca No. 2$1,200-2,500 \mathrm{lbs} /$ acre.

Postmining: Seneca No. $2-1,000-4,500 \quad$ lbs/acre (seeded); mainly alfalfa, and crested and intermediate wheatgrasses.

Premining: Sandy loam and Premining: Pinyon-juniper, big sagebrush, and Premining: Probably No data. clay loam. grasses, alone and intermixed. diverse.

Postmining: Mixed coal fines Postmining: On lower, north-facing slopes, some and shale. colonization by sagebrush and ricegrass; otherwise bare. On leveled oldest (approximately 50 years) workings, heavy sagebrush growth.
Postmining: Mostly limited to plants tolerant of high soil temperature. 


\begin{tabular}{|c|c|c|c|c|c|}
\hline Yine and laxation & $\begin{array}{l}\text { Mining } \\
\text { Beginning date } \\
\text { Acres mined }\end{array}$ & $\begin{array}{l}\text { Reclamation } \\
\text { Beginning date } \\
\text { Acres rectaimed }\end{array}$ & Land Use & Climatic Factors & $\begin{array}{l}\text { Topography } \\
\text { Elevation, in feet }\end{array}$ \\
\hline $\begin{array}{l}\text { 13. HELCO AND } \\
\text { REMINGER MINES } \\
\text { Huntington, Emery } \\
\text { County, central Utah } \\
\text { USGS } 15^{\prime} \text { maps: } \\
\text { Hiawatha } \\
\text { lat } 39^{\circ} 24^{\prime} \mathrm{N} \text {; } \\
\text { long } 111^{\circ} 08^{\prime} \mathrm{W} \text {. } \\
\text { T.16 S., R.7 E. }\end{array}$ & $\begin{array}{l}\text { Underground: } \\
\text { Closed since } \\
\text { 1950's. } \\
\text { Few acres each, } \\
\text { on hillsides. }\end{array}$ & $\begin{array}{l}\text { Trash removal, } \\
\text { 1975-77; } \\
\text { some grass } \\
\text { seeding. }\end{array}$ & $\begin{array}{l}\text { Premining: } \\
\text { Wildlife } \\
\text { habitat. } \\
\text { Intended: No } \\
\text { change. }\end{array}$ & $\begin{array}{l}\text { Growing season } \\
\text { (avg frost-free days): } \\
132 \text { (estimate). } \\
\text { Temperature ( }{ }^{\circ} \mathrm{F} \text { ): } \\
\text { mean annual: } 46 \\
\text { (estimate) } \\
\text { range: No data. } \\
\text { Precipitation (inches): } \\
\text { mean annual: } 14 \\
\text { (estimate) } \\
\text { range: No data. }\end{array}$ & $\begin{array}{l}\text { Steep, rocky cliffs and } \\
\text { narrow canyons with } \\
\text { slopes of } 50 \text { to } 60 \\
\text { percent. Gullying and } \\
\text { rock slides. } \\
7,450\end{array}$ \\
\hline
\end{tabular}

\begin{tabular}{|c|c|c|c|}
\hline 14. NAVAJO MINE & 1963 & Unknown. & Premining: \\
\hline Fruitland, San Juan & & & Wildlife and \\
\hline $\begin{array}{l}\text { County, north- } \\
\text { western New Mexico }\end{array}$ & $\begin{array}{l}5,312 \text { acres } \\
\text { through } \\
1978 .\end{array}$ & $\begin{array}{l}3,548 \text { acres } \\
\text { reshaped; } \\
2,173 \text { acres }\end{array}$ & $\begin{array}{l}\text { livestock } \\
\text { range. }\end{array}$ \\
\hline $\begin{array}{l}\text { USGS } 71^{\prime} \text { ' maps: } \\
\text { Fruitland } \\
\text { lat } 36^{\circ} 42^{\prime} \mathrm{N} \text {; ; }\end{array}$ & & $\begin{array}{l}\text { seeded } \\
\text { through } 1978 .\end{array}$ & $\begin{array}{l}\text { Intended: No } \\
\text { change. }\end{array}$ \\
\hline $\begin{array}{l}\text { long } 108^{\circ} 26^{\prime} \mathrm{W} . \\
\text { T. } 28-29 \text { N., R.15-16 W. }\end{array}$ & & & $\begin{array}{l}\text { Livestock and } \\
\text { hunting ex- } \\
\text { cluded dur- } \\
\text { ing reclama- } \\
\text { tion. }\end{array}$ \\
\hline
\end{tabular}

\section{Growing season}

(avg frost-free days): 139.

Temperature $\left({ }^{\circ} \mathrm{F}\right)$ : mean annual: 52 range: -34 to 104 .

Precipitation (inches): mean annual: 6 range: 2 to 18 ; 50 percent from July through October. Sprinkler irrigation for 2 years, up to 10 in. 1st year; $2 \frac{1}{2}$ inches $2 \mathrm{~d}$ year.
Flat mesas, very low relief. Extensive, low, undulating, graded spoils; some abandoned, entrenched access roads to operating pits and ash disposal areas. Minor rilling due to irrigation operations.

$5,220-5,400$

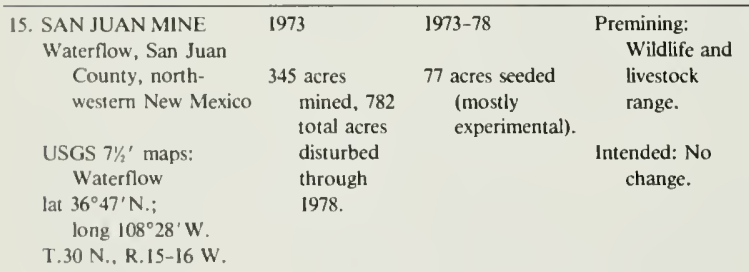

Growing season

(avg frost-free days): 139 .

Temperature $\left({ }^{\circ} \mathrm{F}\right)$ : mean annual: 52 range: 0 to 100 .

Precipitation (inches): mean annual: 7.1 range: 4 to 18 ; 50 percent from July through October. Sprinkler irrigation used for 2 years.
Generally flat with 2- to 4 -percent slopes. Spoils flat to undulating, graded to near original contour.

\footnotetext{
'See (ikavon (1979) for published reclamaion data on specific munes.
} 
Premining: Stony, sandy Premining: Spruce, fir, aspen mixed with pinyon- Premining: Very rich No data. loam grading to clay. juniper and associated shrubs (mainly sagebrush) and grasses (mainly cheatgrass and ricegrass).

flora where two ecotypes meet.

Postmining: Coal fines mixed with rock wastes and premining soils.
Postmining: Sparsely naturally revegetated; some Postmining: On coal ricegrass and mahonio repens on coal fines. fines, very limited.
Premining: Thin clay to silty clay, loam, and sand; sodium absorption ratio 40; $\mathrm{pH}$ 6.1-8.2. Range site types include badlands, sandy, sandy saline, shaly saline, choppy sands, saline lowlands, calcareous, and thin breaks.

Postmining: Spoil is sand, broken sandstone, and shale in three broad types: mostly sand, mostly clay, or a mixture of both.
Premining: Alkali sacaton, galleta, sand dropseed, Premining: Reported to sandhill muhly, broom snakeweed, Mormon-tea, greasewood, shadscale, and fourwing saltbush.

Postmining: Under irrigation, perennial vegetation provides 10 to 15 percent cover. Ground layer of some grasses and annual plants. Scattered Indian ricegrass, wheatgrasses, sand dropseed, galleta, and giant dropseed. Density and composition vary with spoil type. Fourwing saltbush appears to be thinning out with the discontinuation of irrigation.

\author{
Premining: 29-10l lbs/acre \\ (overgrazed); 250-500 \\ lbs/acre potential. \\ Postmining: In 1975, 41-614 \\ $\mathrm{lbs} /$ acre for irrigated areas.
}

of annuals and perennials, both palatable and nonpalatable.

Postmining: 15 to 20 species observed.
Premining: Silty clay loam to
sandy loam; substrate is
sandstone and siltstone;
sodium absorption ratio
as high as 27; pH 7.8-8.2.
Postmining: Spoils are shale
and (or) sandstone.
Problems of high sodium
and high clay content are
partly solved by 12 -in.
topsoiling in two separate
applications (4 in. and 8
in).
Premining: Predominantly open grassland; galleta, Premining: Over 10 alkali sacaton, Indian ricegrass, sand dropseed, fourwing saltbush, and shadscale saltbush.

Postmining: Ground cover generally 5 percent; Russian-thistle and sand dropseed dominant. Fourwing saltbush has become established providing 10 to 15 percent cover. Nonirrigated areas have not revegetated. 1978-79 seedings successful.
Premining: 139-195 lbs/acre for grasses and shrubs.

Postmining: 1973-77, Postmining: No data. four species in seed mix; 1978, 10 species in seed mix. 


\begin{tabular}{|c|c|c|c|c|c|}
\hline Mtae and location & $\begin{array}{l}\text { Misnng } \\
\text { Begunung date } \\
\text { Acres mined }\end{array}$ & $\begin{array}{l}\text { Revlamation } \\
\text { Bcginning date } \\
\text { Actes reclaimed }\end{array}$ & Land Use & Climatic Factors & $\begin{array}{c}\text { Topography } \\
\text { Elevation, in feet }\end{array}$ \\
\hline $\begin{array}{l}\text { 16. BLACK MIESA AND } \\
\text { KAYENTA MIINES } \\
\text { Kayenta, Navajo Reser- } \\
\text { vation, Arizona } \\
\text { USGS } 71^{\prime} \text { ' maps: } \\
\text { Long House Valley } \\
\text { Great Spring } \\
\text { lat } 36^{\circ} 32^{\prime} \mathrm{N} \text {.; } \\
\text { long } 110^{\circ} 24^{\prime} \mathrm{W} \text {. } \\
\text { lat } 36^{\circ} 29^{\prime} \mathrm{N} . ; \\
\text { long } 110^{\circ} 23^{\prime} \mathrm{W} \text {. } \\
\text { T.35-36 N., R. } 18 \mathrm{E} \text {. }\end{array}$ & $\begin{array}{l}1970 \text { for both. } \\
\text { Black Mesa: } \\
\text { 1,485 acres } \\
\text { to } 1978 . \\
\text { Kayenta: } 1,434 \\
\text { acres to } \\
1978 .\end{array}$ & $\begin{array}{l}\text { Black Mesa: } \\
818 \text { acres } \\
\text { reshaped, } 200 \\
\text { acres fenced } \\
\text { and seeded. } \\
\text { Kayenta: } 494 \\
\text { acres } \\
\text { reshaped, no } \\
\text { revegetation. }\end{array}$ & $\begin{array}{l}\text { Premining: } \\
\text { Wildlife and } \\
\text { bivestock } \\
\text { range. } \\
\text { Intended: No } \\
\text { change. } \\
\text { Fenced; } \\
\text { livestock ex- } \\
\text { cluded from } \\
\text { planted areas } \\
\text { during } \\
\text { reclamation. }\end{array}$ & $\begin{array}{l}\text { Growing season } \\
\text { (avg frost-free days): } 145 \text {. } \\
\text { Temperature ( }{ }^{\circ} \mathrm{F} \text { ): } \\
\text { mean annual: } 53 \\
\text { range: }-18 \text { to } 105 \text {. } \\
\text { Precipitation (inches): } \\
\text { mean annual: } 9.5 \\
\text { range: } 6.8 \text { to } 11.5 ; \\
40 \text { percent from July } \\
\text { through October. } \\
\text { Evaporation rate is very } \\
\text { high; most moisture } \\
\text { evaporates before pene- } \\
\text { trating soil. }\end{array}$ & $\begin{array}{l}\text { Hilly, dissected mesas } \\
\text { with 2- to } 15 \text {-percent } \\
\text { slopes. Rolling, } \\
\text { graded spoils blended } \\
\text { to unmined areas. } \\
\text { Water impoundments } \\
\text { and moisture basins. } \\
6,500-6,600\end{array}$ \\
\hline $\begin{array}{l}\text { 17. MCKINLEY MINE } \\
\text { Mckinley County, north- } \\
\text { western New Mexico } \\
\text { USGS } 71^{\prime} \text { ' maps: } \\
\text { Zith Tusayan Butte } \\
\text { NE } \\
\text { Tse Bonita School } \\
\text { Hunters Point } \\
\text { Sarnson Lake } \\
\text { lat } 35^{\circ} 37^{\prime} \text { N.; } \\
\text { Jong } 109^{\circ} 00^{\prime} \mathrm{W} \text {. } \\
\text { T.16-17 N., R.20-21 W. }\end{array}$ & $\begin{array}{c}1962 \\
1,344 \text { acres } \\
\text { through } \\
1978 .\end{array}$ & $\begin{array}{l}\text { 1,185 acres } \\
\text { reshaped } \\
\text { through } 1978 . \\
\text { Prior to } 1975, \\
\text { crested } \\
\text { wheatgrass } \\
\text { used on } \\
\text { reshaped } \\
\text { spoil. }\end{array}$ & $\begin{array}{l}\text { Premining: } \\
\text { Wildlife and } \\
\text { livestock } \\
\text { range. } \\
\text { Intended: No } \\
\text { change. }\end{array}$ & $\begin{array}{l}\text { Growing season } \\
\text { (avg frost-free days): } 120 \text {. } \\
\text { Temperature }\left({ }^{\circ} \mathrm{F}\right): \\
\text { mean annual: } 48 \\
\text { range: }-35 \text { to } 95 \text {. } \\
\text { Precipitation (inches): } \\
\text { mean annual: } 10.4 \\
\text { range: } 9 \text { to } 12.5 ; \\
40 \text { percent from July } \\
\text { through September. }\end{array}$ & $\begin{array}{l}\text { Benches, mesas, rocky } \\
\text { breaks with } 2 \text { - to } \\
8 \text {-percent slopes. } \\
\text { Spoils graded to near } \\
\text { natural contours to } \\
\text { blend with adjacent } \\
\text { landforms; natural } \\
\text { drainage patterns } \\
\text { reconstructed. Minor } \\
\text { erosion controlled by } \\
\text { contour furrowing. } \\
7,000-8,000\end{array}$ \\
\hline
\end{tabular}

\footnotetext{
'See Gieason (1979) for published reclamation data on specific mines.
} 
Premining: 1nfertile fine san- Premining: Pinyon-juniper woodland; big sage- Premining: Probably dy soils and sandstone rock outcrops; sodium absorption ratio is 1 on unmined topsoil and 10 on reshaped spoils; $\mathrm{pH}$ $6.9-8.0$; no toxic conditions.

Postmining: Loamy clays and sandy clay loams containing stone: brush, snakeweed, fourwing saltbush, rabbitbrush, galleta, Indian ricegrass, blue grama, broom snakeweed, sand dropseed, fescue, and prickly pear.

Postmining: Only revegetated area (1978) limited to Russian-thistle with scattered grasses, sweetclover, alfalfa, Indian ricegrass, blue grama, and fourwing saltbush due to overgrazing by sheep. Areas now fenced.
Premining: 12-432 lbs/acre for native vegetation (excluding pinyon-juniper). Heavily overgrazed, potential not estimated.

Postmining: Revegetation limited; area subject to severe overgrazing. Premining: Clay to silt
loams; sodium absorption ratio as high as 19 ; pH 6-7 on unmined areas, $5.3-8.1$ on mined areas.

Postmining: Mix of rubble, soil, and rocks; blown-on straw mulch. Topsoiling began in 1978 .
Premining: Primarily pinyon-juniper, big sagebrush, Premining: 163 species. blue grama, galleta, western wheatgrass, Indian ricegrass, and needleandthread.

Postmining: Fourwing saltbush, sagebrush, and western and crested wheatgrasses. Some saltbush are volunteer seedlings.
Postmining: Natural succession on raw spoils; 72 species $(34$ maximum on one plot), mostly introduced annuals or native herbaceous plants and a few saltbush; Russianthistle dominant. Initially one species planted (crested wheatgrass). As of 1978, new seed mixtures being tried.
Premining: No data.

Postmining: Highly variable with location and aspect-north slopes produce much more than south slopes. 


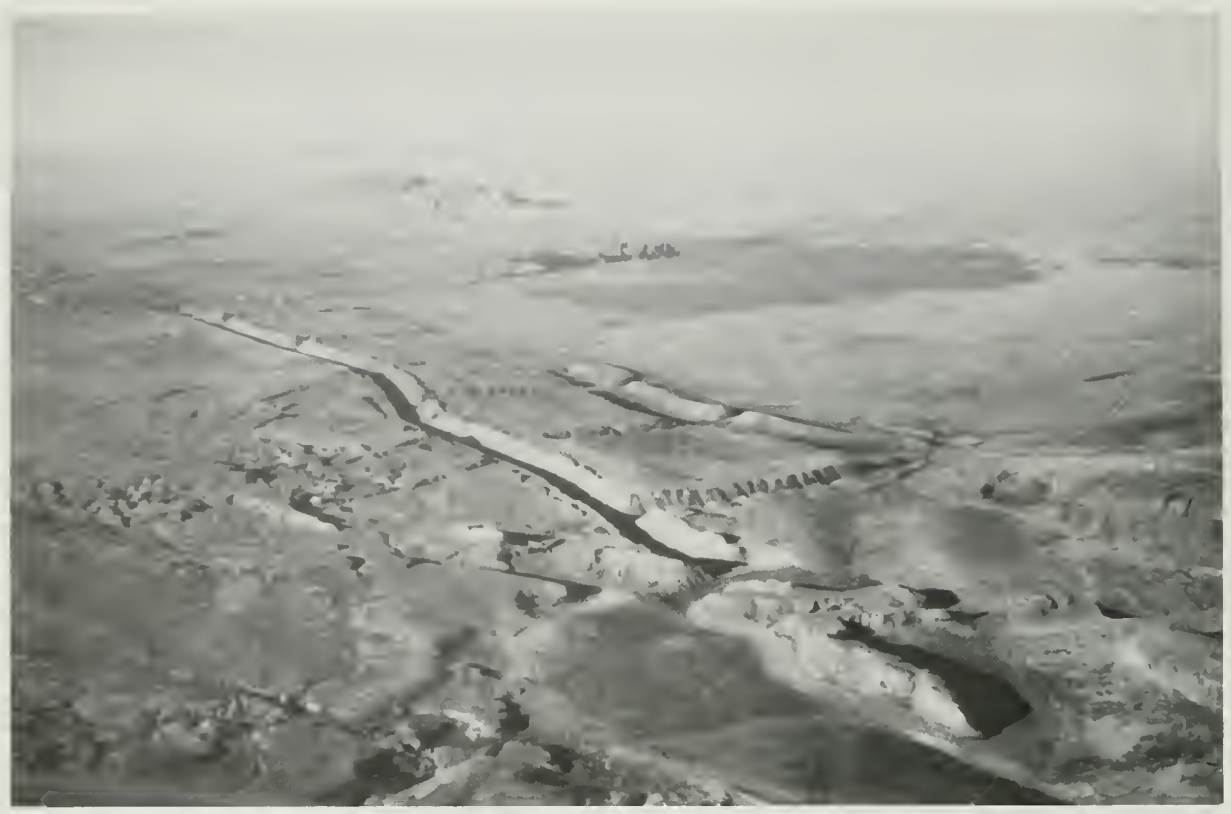

FIGURE 5.-Aerial photograph of the Navajo surface mine in New Mexico, showing mining operations proceeding 10 miles toward the south. A single 16-foot bed in the north and four thin coalbeds in the south are being mined. The associated Four Corners powerplant and its cooling basin are in the center. This mine is in an area of less than 10 inches of raintall per year, and irrigation is being used to reestablish vegetative cover on the graded spoils. Haul roads that are perpendicular to the working cut were not completely covered because of continued mining. November 1978.

and scale. What seems acceptable in laboratory studies or on experimental plots at mines may not be successful for a large area over long periods of time, and results can vary widely with changes in local reclamation techniques and with climatic factors. These problems were generally recognized in one of the most comprehensive field examinations to date on the revegetation potentials of western coal mines (Packer and others, 1981). The authors of that study examined 28 of the major western coal mines in 1976 and 1977; until 1976, revegetation had been attempted at only 36 western coal mines. Although expressing qualified optimism for success, they indicated that a reassessment in 5 or 10 years would be needed. Their study included maps of the Western States that show locations of surface minable coal, soil associa. tion types, potential natural vegetation types, the average annual precipitation, and length of growing season.

\section{REGIONAL CHARACTERISTICS THAT AFFECT RECLAMATION}

\section{LANDFORM AND CLIMATE}

The western coalfields, although extending over almost $18^{\circ}$ of latitude, have some common geographic features. They occur on high plains, dissected plateaus, and mountainous foothills at elevations above 2,000 feet, and many occur at elevations of 5,000 to more than 6,000 feet. The climate is continental and semiarid. The mean annual precipitation (fig. 15) in most areas is between 7 and 15 inches, reaching about 19 inches in the higher elevations in Colorado. Evaporation rates may be three times greater than the precipitation rate. The amount of precipitation varies widely from year to year, and droughts are common. Except in the higher elevations in Colorado and Utah, most of the precipitation occurs in the 


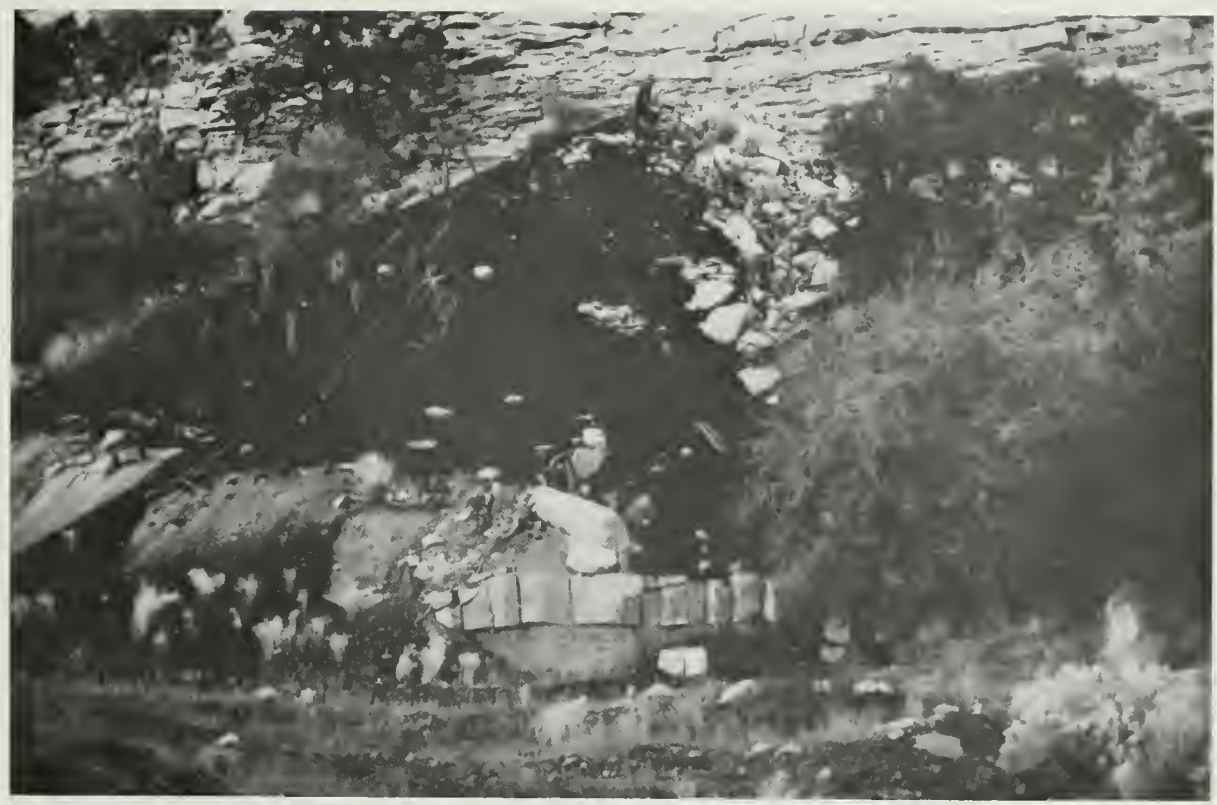

FigurE 6.-Waste coal dumped below the entry of the abandoned Reminger coal mine in Utah. A single 8- to 10-foot coalbed was mined and transported to bins through wooden chutes. The mine ceased production in the late 1940 's. This scene is typical of the entrances of old abandoned underground coal mines. Sparse clumps of ricegrass grow on the base of the slack coal. The woody debris consists mainly of juniper trees that were killed by landslides. These steep slopes are not conducive to revegetation. The rockfall has partly covered the old access road in the foreground. October 1978.

summer months, usually during short, highintensity storms. Mean annual temperatures (degrees Fahrenheit) generally are in the 40's, extending into the 50's in New Mexico and Arizona. The range in latitude and elevation is reflected in changes in evaporation rates (fig. 16) which generally increase from north to south and, more specifically, in the length of the growing season, which can be as short as 60 days (in Colorado) and as long as 145 days (in Arizona).

\section{GEOLOGY AND SOILS}

Most of the western coalbeds are thick and relatively flat, have a low sulfur and ash content, and are of similar geologic age-Cretaceous through early Tertiary. They are usually overlain by shales and sandstones, some of which have high salinity and (or) sodium content. Sodic swelling clays commonly characterize the over- burden of the Fort Union Formation in North Dakota and Montana. Soils vary from clayey to sandy depending on the underlying rock type. They are usually shallow and contain thin "topsoil" layers and limited organic matter. Subsoils are typically finer textured than the surface soils and often have accumulations of calcium carbonate or calcium sulfate (gypsum). Soils having high sodium content may be present in depres. sions and where soils have formed from shales. Moderate salinity in soils is common.

\section{VEGETATION}

The natural vegetation in the western coal region is typically western zonal, responding to precipitation, latitude, altitude, and aspect. Overall, plant species and density are closely related to soil characteristics and climate. The lands to be mined are grassland, shrubland, or 


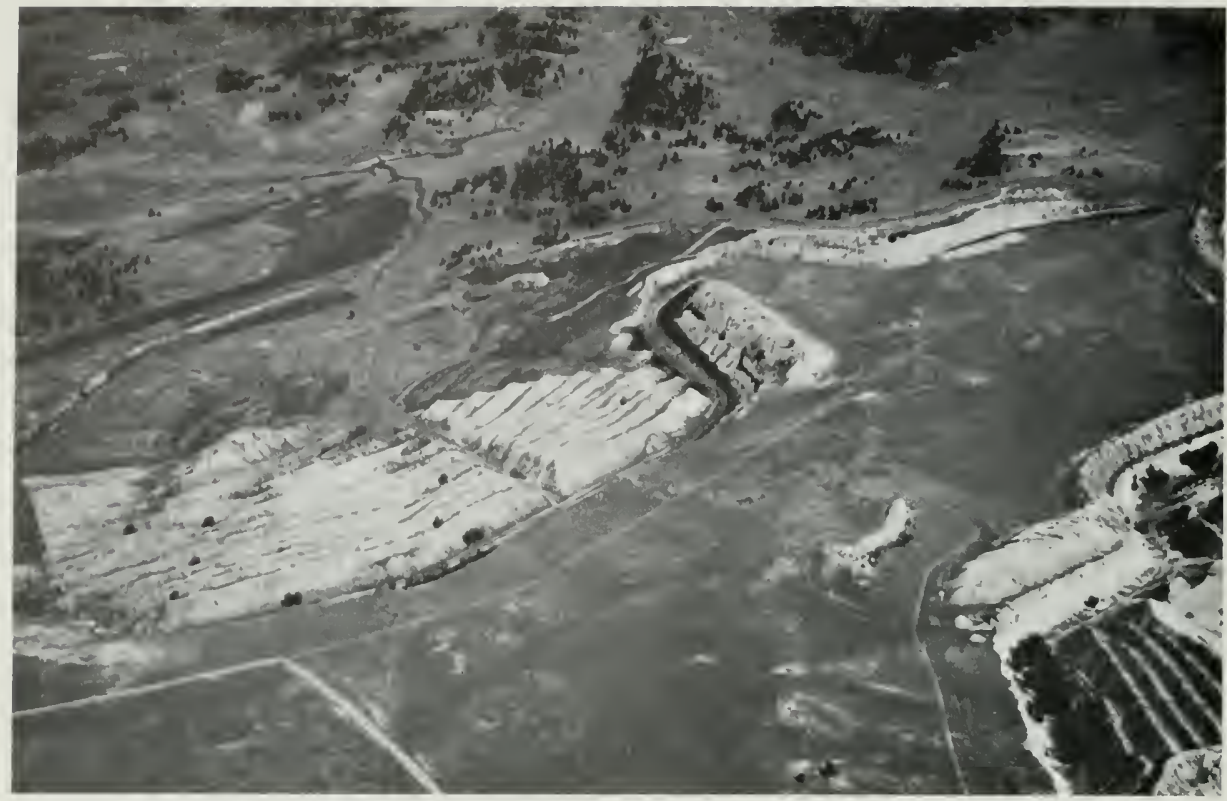

FIGURE 7.- Aerial photograph showing rows of abandoned spoil piles at the Rosebud mine in Montana. In 40 years, little natural vegetation has encroached on the steep slopes. Large cottonwoods are scattered in the low places; a final box cut is filled with water and stocked with fish, and it provides refuge for some migratory waterfowl. Highwalls now must be reduced under the new State and Federal laws. July 1978.

mixtures of the two and, in places, grade into open woodland. The trees are largely pinyon and juniper in the south and ponderosa pine in the north. Some coal lands in the central part of the region are covered by mixed aspen-oak brush and mixed high-altitude shrubs called "mountain brush." Spruce-fir forests cover the land over some of the underground coal mines of Utah and Colorado.

The vegetation is, for the most part, native and wild and is interrupted only occasionally by pasture and cropland. North Dakota is the major exception; there, much of the land overlying areas of coal is in grain or hay crops. By far, the dominant use of land is for livestock range. The Federal lands are managed, under the multipleuse policy, for both rangeland and for wildlife habitat, and the planned postmining uses are basically the same as those that existed before mining whether the land is owned privately or by the Government.

\section{SITE-SPECIFIC CHARACTERISTICS THAT AFFECT RECLAMATION}

\section{INTRODUCTION}

Reclamation is best planned and implemented on a case-by-case basis. No single planning methodology is suitable for all mines. Although the widest differences are regional, adjacent mines within the same district or even parts of a single mine may present distinct reclamation challenges, and individual adjustments to the differences in soil materials, elevation, slope, aspect, time and amount of precipitation, and growing season are commonly needed. Ignorance of site-specific characteristics of mined areas has often hampered revegetation, caused inaccurate statements, and necessitated excessive expendituies. 


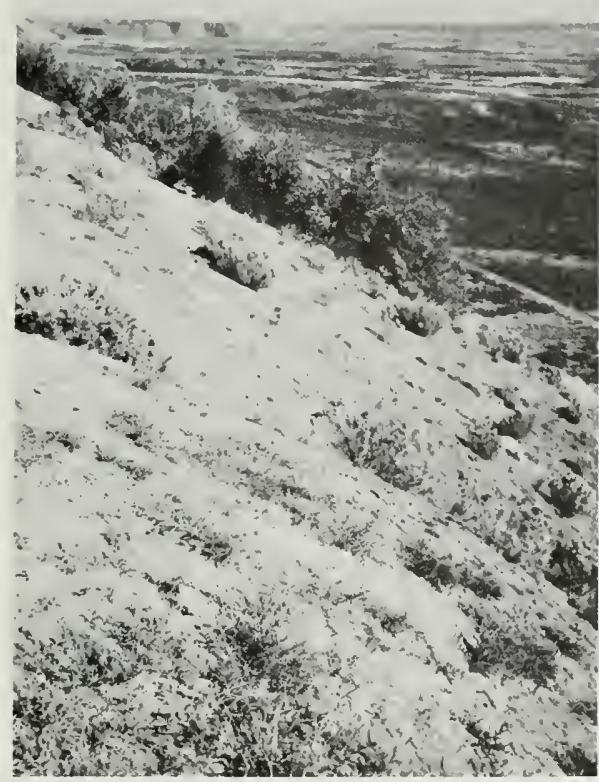

FIGURE 8.-Natural revegetation on the 19-year-old mine waste dumps at the Elkol-Sorensen mine in Wyoming. The waste material, sloping about $40^{\circ}$, ranges from boulders to find sand. The foreground shrubs are mostly rabbitbrush. The denser trees and shrubs on the slope were planted by the University of Wyoming on an experimental basis. Of these, the Russian-olive is doing best. Although sparsely vegetated, these east-facing slopes were some of the best revegetated steep waste piles observed. October 1978.

Examination of many western surface coal mines will disclose experimental test plotssome active but most seemingly abandoned or waiting to be reevaluated for long-term results. These experimental test plots include plantings and surface manipulations conducted or guided by various Federal agencies such as the U.S. Forest Service, Soil Conservation Service, and the Bureau of Land Management, and by State agencies and local universities. The Forest Serv. ice Intermountain Forest and Range Experimental Station, and later the Forest Service SEAM program, sponsored many experiments in the northern Great Plains area, including experi- ments at the Decker mine (Richardson and others, 1975), one of the oldest of its kind and one that continues to be monitored. Quantitative analyses of results of most of these experiments, however, were not available at the time of this study, in part because of changes in mining reclamation personnel. Some plantings on the test plots bear little resemblance to what is planted on adjacent lands because they were made for general research purposes rather than for the specific needs of a particular mine. In some mined areas, nonnative species are predominant.

At some mines, experimentation on a large scale is being done by mining companies. These experiments are conducted to assess the usefulness of specific reclamation techniques. Results of these experiments have been reported on at various symposia and in various publications that form the basis for recommendations made by the SEAM program and by Cook and others (1974).

Through the research programs identified above, the significant parameters affecting reclamation success have been defined. As indicated earlier, revegetation procedures involve few deviations from what good horticultural and ecological sense would have predicted. Parameters believed to be significant to revegetation success are briefly discussed below in terms of both their effects on the total reclamation process and on some operating procedures used to enhance their positive effects. Because reclamation deals with an interrelated system of plants, soils, topography, and climate, a procedure used to modify any one parameter is likely to affect all. The procedures are discussed under the parameter heading believed to be the most appropriate.

\section{CLIMATE}

Of all the parameters affecting reclamation, climate is the most important and the least controllable. The principal climatic factors affecting plant growth are precipitation, temperature, and wind. These three, separately or in combination, affect the germination, growth, and distribution of plant life. Although temperature extremes and the number of frost-free days, namely, the growing season, affect the time and rate of growth 


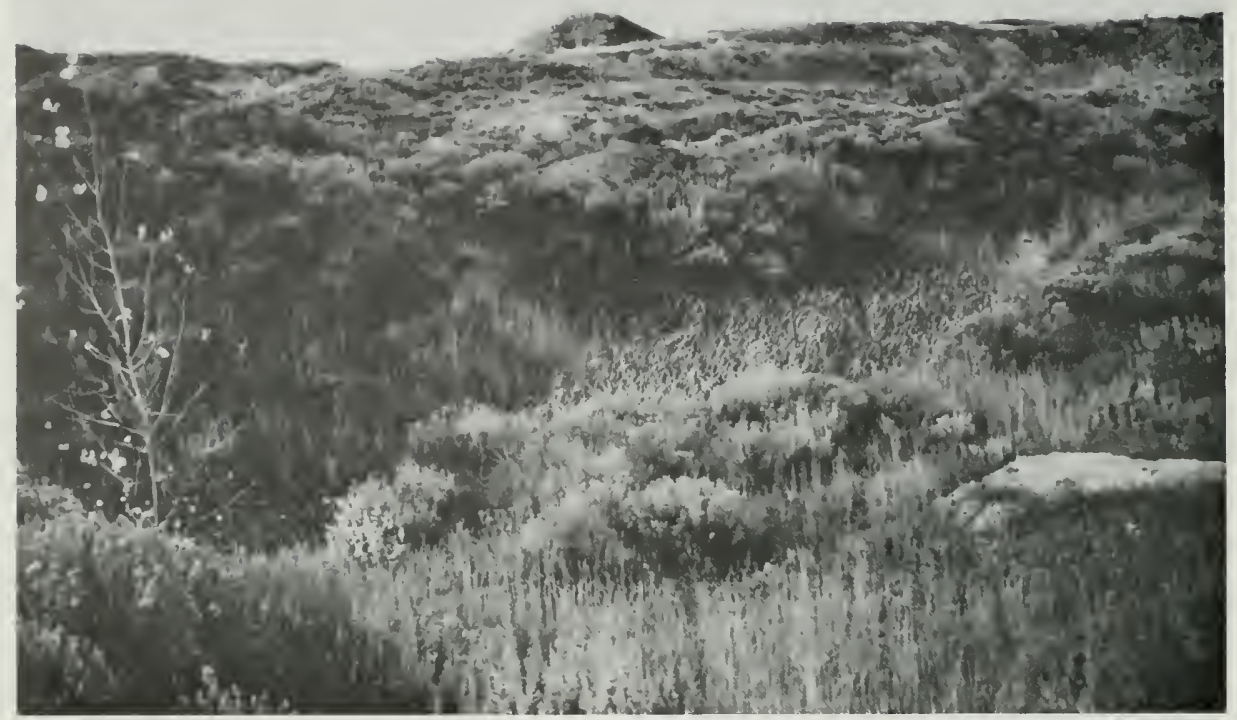

FIGURE 9.-Old orphaned spoils naturally revegetated during the past 35 years at the Indian Head mine in North Dakota. The steeper, clay-rich slopes of this spoil (right background) are sparsely vegetated because of their high sodium content. This area has abundant wildlife, including deer. October 1978.

and the yield (biomass) potentials, and although wind may bury seeds and plants, abrade them, or expose them to dessication, the amount of moisture available to plants is the governing factor in successful revegetation. Because of the high evaporation rates in the western coal areas, the availability of soil moisture is the critical element in seed germination, early growth of plants, establishment of transplants, and continued growth. A detailed statistical examination of western coal reclamation potentials using multiple regression analysis confirmed that the amount of precipitation and the length of the growing season are highly significant factors (Packer and others, 1981).

Although there is no realistic way to modify adverse effects of a microclimate except by adding moisture through irrigation, a variety of procedures have been used to minimize unfavorable conditions at the microclimatic level. Ground temperatures that affect seed germination and growth can be modified by using mulches or temporary cover crops, by altering slope aspect, or by establishing other shading devices such as rows of trees and shrubs, rock piles, or snow fences. These procedures also reduce wind and water erosion and conserve soil moisture. More passive approaches can also be taken. For example, seed that first needs a cold period to germinate can be sown in the fall in accordance with normal seasonal temperature changes.

\section{SOIL MOISTURE}

Ensuring adequate soil moisture for plants has been approached in several ways, not only to enhance seed germination and early seedling survival, but also to provide deeper, continued sources of moisture as plants mature. Two studies Wyatt and others, 1980; Schumacher and others, 1977, p. 16) have indicated that most roots are in the upper 5 to 6 feet of mine spoils. 


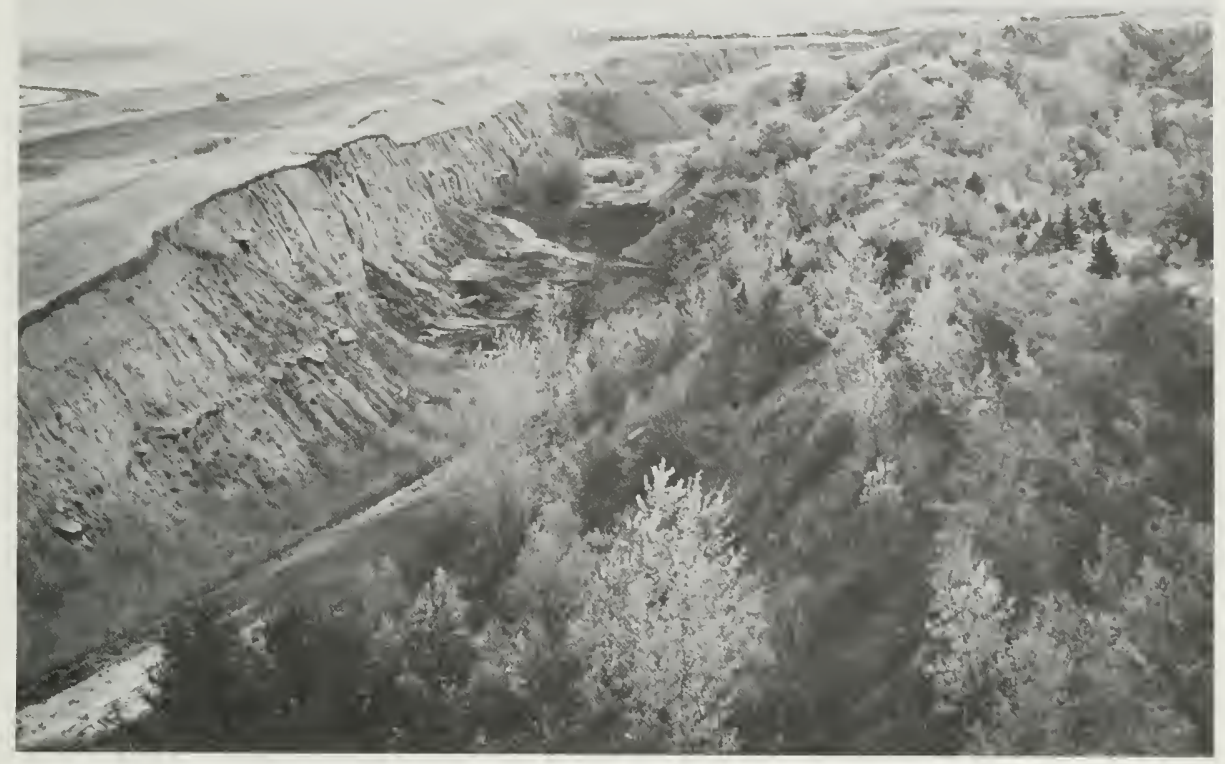

Figure 10.- The North Beulah mine in North Dakota. Revegetated orphaned spoils (right) provide an excellent habitat for wildlife. The plants have been browsed extensively by deer. The area, which is near the Indian Head mine, was planted by conservation groups to serve as a refuge and is a good example of alternatives for successful reclamation of surface-mined land. The premining surface is seen at the upper left. The highwall and final cut remain nearly bare of vegetation. October 1978.

Sowing is usually done to take advantage of peak precipitation and soil moisture periods. To improve soil moisture absorption and retention and to protect newly germinated seeds, straw or hay mulch is commonly added to the new soil surface. In most places such mulching materials must be partially buried to keep them from blowing away (Packer and Aldon, 1978, p. 434). A variety of slurried spray-on mulching materials is available (Kay, 1978, p. 471-478), and some have been tested and used in mine reclamation (fig. 17). Mulching is now a general requirement, but it can be omitted under certain circumstances (U.S. Office of Surface Mining, 1979, p. 15413). Experience has shown that mulches may not be advantageous everywhere. They may temporarily lead to a tying up of soil nutrients and thus create a need for additional fertilizers (Rod Gabehart, Western Coal Co., San Juan Mine, oral communication, 1978).

Techniques, including construction of ter-

races (fig. 18) and small scattered depressions and use of continuous deep grooves or furrows along the contour of the land (figs. 17, 18, 19, 20, and 21) have been developed to increase the amount of moisture stored in reworked soils and spoils (Jensen and Schafer, 1979). The latter technique, termed "deep chiseling," is effective in concentrating water in the root zone. In the windy Hanna Basin area of Wyoming, chiseling was also effective in reducing wind destruction of small seedlings which grew only in the 12inch deep grooves. Most of these manipulation techniques serve the dual purpose of reducing the amount and speed of water runoff and therefore are useful in erosion control. According to Jensen and Schafer (1979, p. 129), both replaced soils and spoils tend to have reduced capacity to absorb water because of a reduction in porosity. Topsoiling, however, has proven to be an effective procedure for increasing water infiltration because the topsoil layer acts like a sponge. 


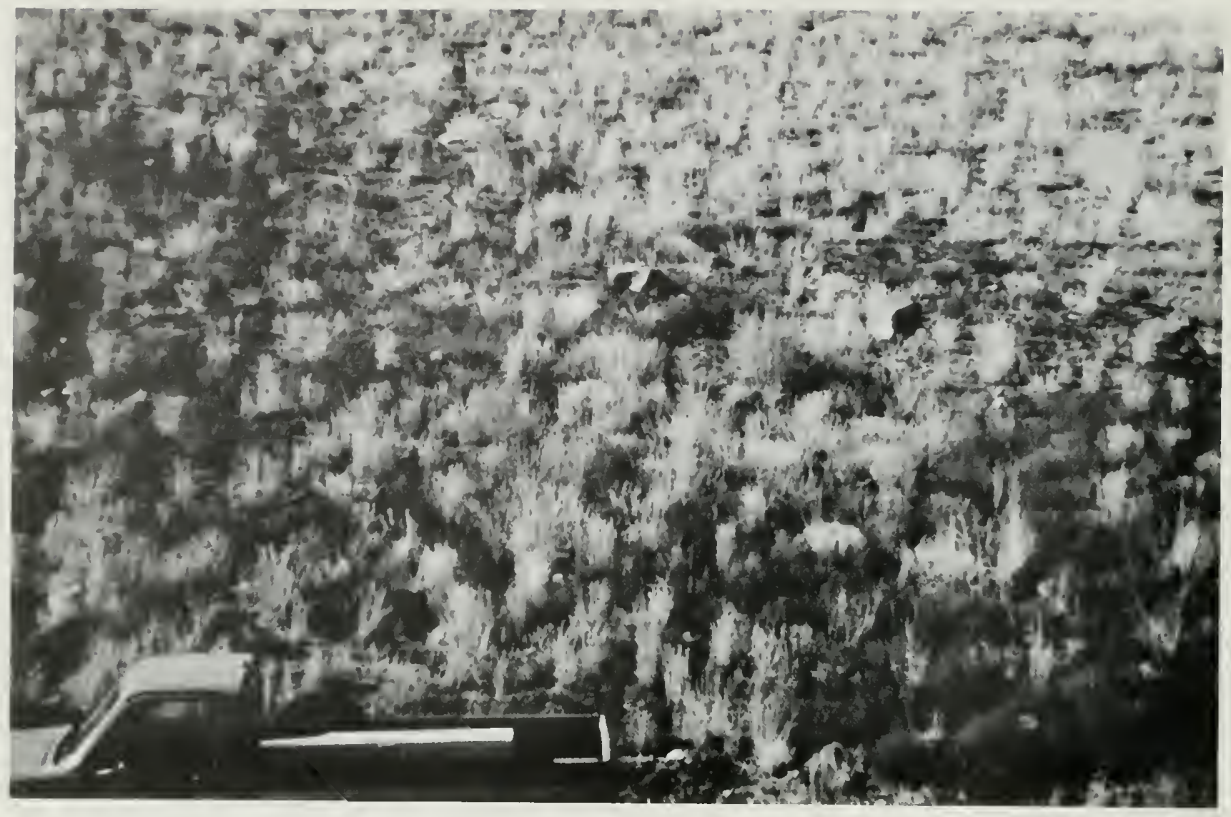

FIGUAE 11. - Second season of revegetation by seeding at the McKinley mine in New Mexico. The area was seeded with western and crested wheatgrasses (light clumps) and planted with seedlings of fourwing saltbush (dark clumps). Some fourwing saltbush has reseeded on this slope. Thin topsoil was used; some undisturbed areas at the mine site have little if any soil to be saved for topsoiling. This land will be returned to use as range. Note the spacing of the saltbush in this planting, which was made without irrigation; the plants developed in adjustment to natural moisture availability. November 1978.

Irrigation allows planting over a greater time period and assures the highest percentage of seed germination and initial plant survival. Irrigation was observed as an operating procedure only at the Navajo and San Juan mines of New Mexico where powerplant cooling water is readily available. It has also been experimentally used at other mine reclamation sites (Ries and Day, 1978, p. 509-512). Sprinkler irrigation has proved to be the most satisfactory method under field conditions (Aldon and others, 1976). Irrigation must be scheduled, however, so that when it is discontinued some plants will survive without it (Curry, 1975, p. 36-37). It has been observed that on some irrigated mine spoils in New Mexico, vegetation thinned out significantly after irrigation was stopped.

\section{DROUGHTS}

Droughts occur periodically in the western coalfields. A plant species or plant community that is only marginally established is more sensitive to the stress of overgrazing, insect attack, or drought. Although the first two can be controlled, the climate cannot. Dry years have had disasterous effects on new plantings, but the effects of sustained drought on established mine reclamation plantings have not been documented. There have been no sustained long droughts in the western coal region since the late 1930's (Curry, 1975, p. 40). Following the droughts of that period, however, large changes in plant communities, both native and planted, did occur in a variety of physical situations 


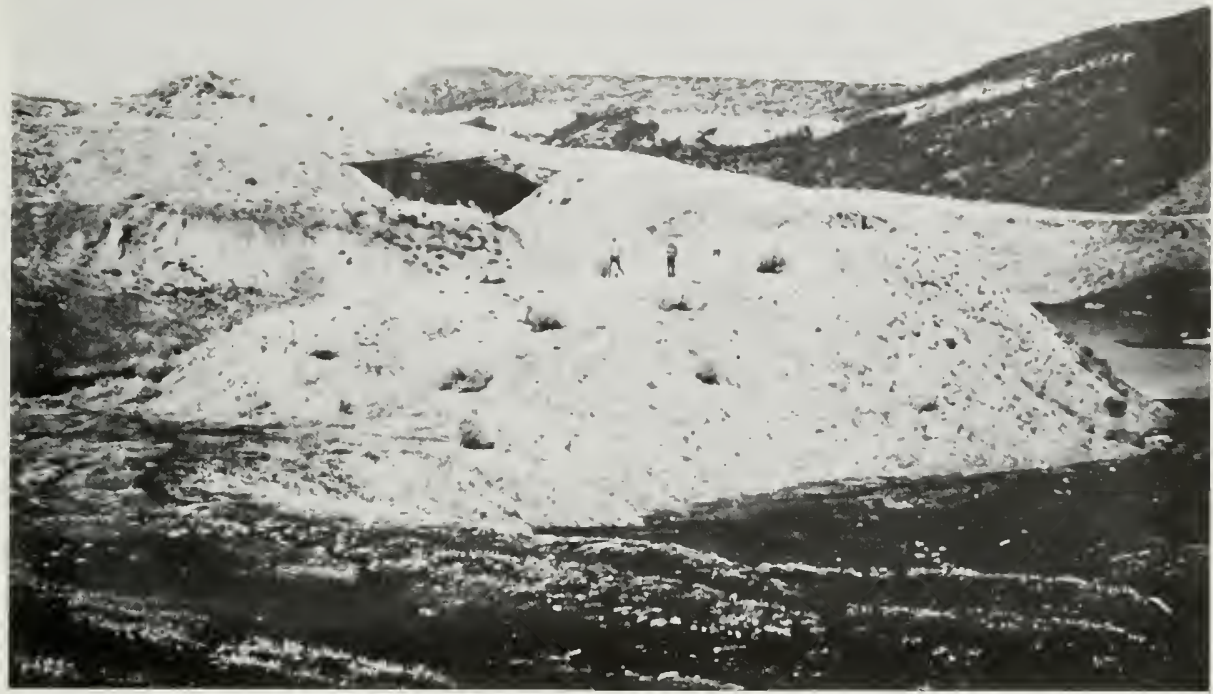

Figure 12.- Natural revegetation on old spoils near the Seneca mines in Colorado. The spoil piles were flattened on the top and then airseeded with grass. The slopes are from $35^{\circ}$ to $40^{\circ}$. The pits at the end of mining trenches are filled with water. They contain small amounts of bullrushes and provide waterfowl habitats. The coal slack (foreground) remains essentially unvegetated. Perennial cover is sparse in contrast to the naturally vegetated slopes in the background, but some shrubs, mainly big sagebrush, have naturally invaded the spoil. Some exotic trees and shrubs were planted on these spoils, and the survivors are doing well. October 1978.

(Thornburg and Fuchs, 1978, p. 414). Some investigators (U.S. Geological Survey and Montana Department of State Lands, 1979, p. II-28-29) think that the high levels of precipitation in the last 10 years in Montana represent the high part of a general 21-year cycle which is expected to decline.

\section{LANDFORM}

The reshaped form of the mined land surface strongly influences the potential for reclamation success. In the past, this surface encompassed the nearly vertical cliffs, or highwalls, which marked the end of the mine pit area (figs. 10 and 22) and mine pits or other depressions that would be perennially or seasonally flooded (fig. 12). Most of the other disturbed ground was composed of piles of spoil that formed coalescing hills and ridges having slopes at the angle of repose of loose material (figs. 3, 7, and 10).

Erosion is greater on steep slopes and on long slopes. On these slopes, seeds or seedlings, even if they can be established, are likely to be washed away or buried by eroding material. Steep slopes are dry even if they are composed of porous material because the rainfall runs off rapidly. Although annuals and sparse clumps of grasses may be observed, stabilizing vegetation is difficult to establish either artificially or naturally on steep slopes (figs. 7 and 23), and they may remain almost bare after 40 years. An almost impermeable surface is formed when slopes are composed of clay, particularly swelling clay. At dry, windy sites, extensive flat areas can also be ultimately unproductive because wind erosion may create shallow depressions in 


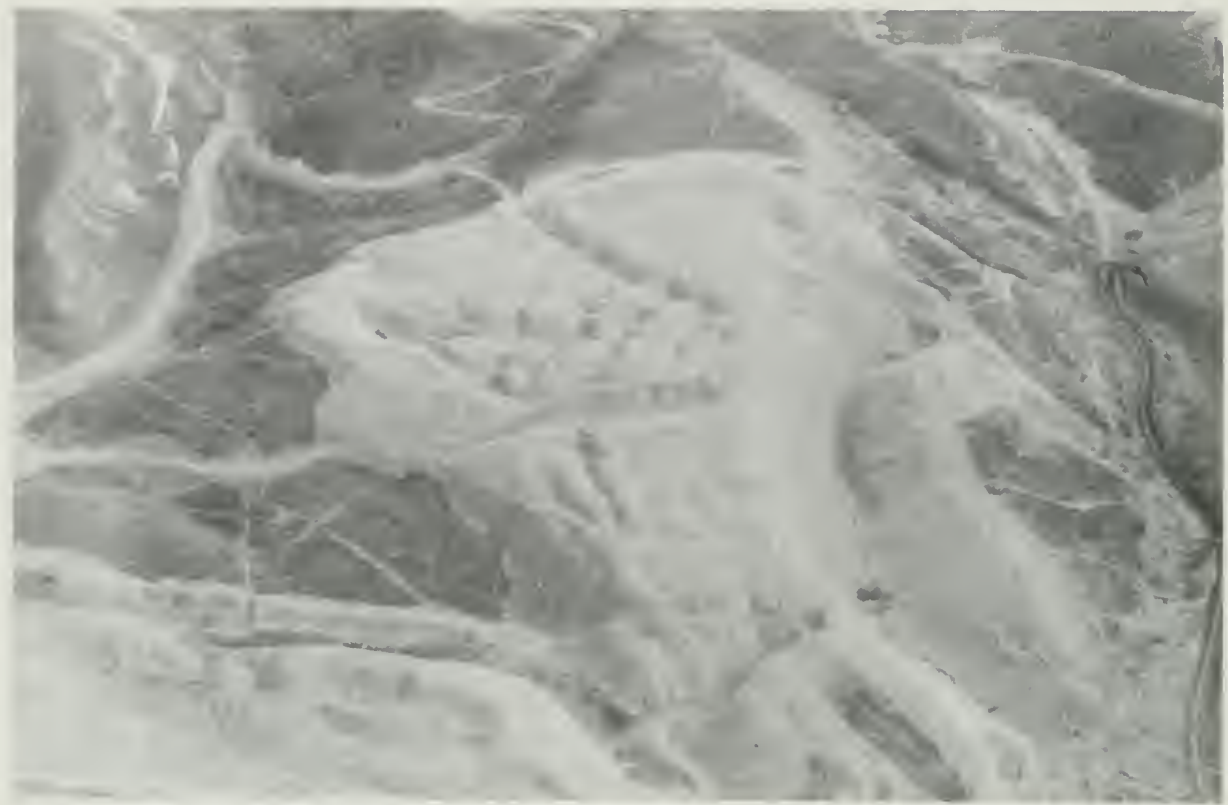

FIGURE 13.-Aerial photograph showing the hummocky surface created by rounding off tops of spoils at an older part of the Seneca mine in Colorado. The graded highwall forms the right side of the draw. These grass-covered stable surfaces contrast sharply with the darker surrounding mountain brushland. October 1978.

which salts accumulate as a result of the evaporation of water.

Aspect, the direction that a slope faces relative to the Sun, also affects plant growth. Even where temperatures are generally cool, average precipitation high, and evaporation low, as in the northwest Colorado coalfields, old south- and west-facing spoil slopes remain bare if they are composed of very coarsely textured materials (Berg, 1975, p. 81). North- and east-facing slopes are cooler, more moist, and therefore are usually easier to revegetate (Jonescu, 1974). South- and west-facing slopes are hotter, drier, and generally more difficult to revegetate (frontispiece A). Seed mixes tailored according to slope aspect have not been widely used in reclamation.

As discussed under the section "Climate," most surface manipulations that help catch and conserve moisture also reduce erosion. Regulations now require that the new surface be generally similar to the premining surface and that the slope of the highwalls be reduced.

\section{OVERBURDEN}

The chemical and physical characteristics of the geologic materials lying above the coal, called overburden, or between coalbeds where more than one bed is to be mined, called interburden, are significant parameters in reclamation success. These characteristics are especially true where spoils comprise all or part of the new growth media, because they control internal drainage and contribute harmful or plantgrowth-limiting materials. In general, the clayey materials present the greatest problems in western reclamation because they are slow to absorb moisture and often have high salt content; these properties are responsible for poor revegetation success at many sites. Sodium-rich expanding clays are particularly adverse for seed germination and growth (fig. 24). Where both sandy and clayey materials are present in the overburden, some successful intermixing can be done in the stripping process (Dollhopf, 1979) to reduce the 


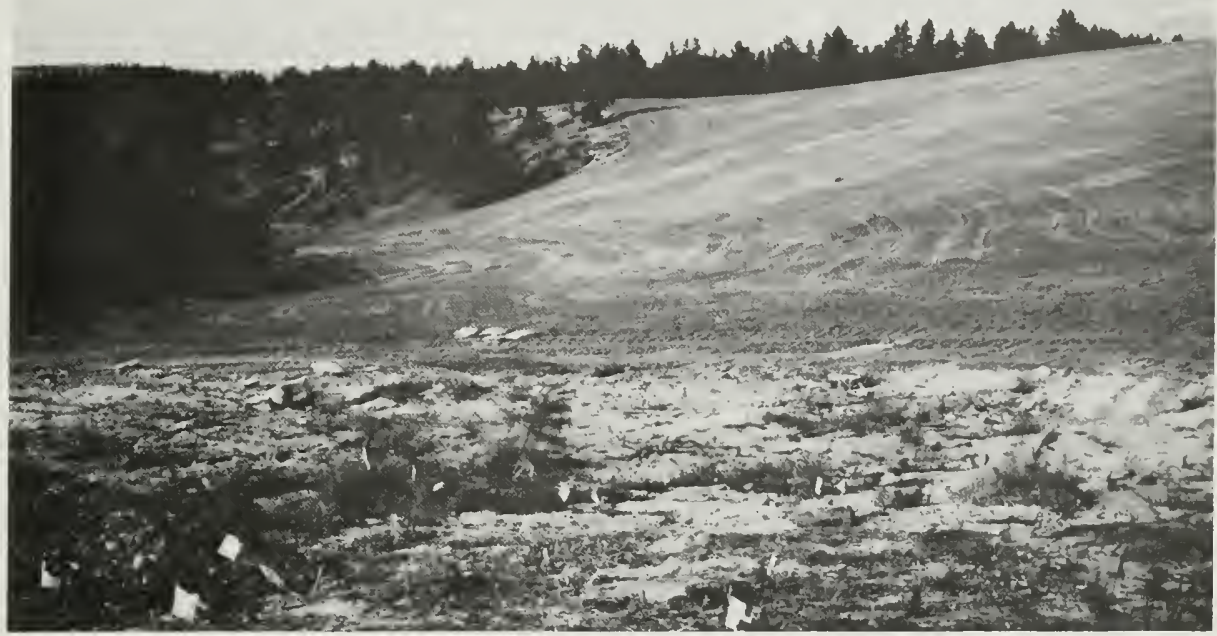

FIGURE 14.- Graded and seeded spoil blending into the natural wooded terrain at the Absaloka mine in Montana. The chiseled, rough surface will accumulate moisture during the winter for germination of the grass seed in the spring. In the foreground, pine seedlings and native transplants were used to reintroduce the natural pine-grassland interface vegetation in this region. Both containerized and bare-rooted seedlings were used. Bare-rooted plants had a lower survival rate. October 1978.

the undesirable characteristics of the clayey materials.

The greatest technical challenge occurs when the entire overburden has a harmful character, such as in the sodic clay sequences in the North Dakota and adjacent Canadian lignite coalfields. in some places in North Dakota, the salvage and replacement of topsoil has been generally effective (Pole and others, 1979); in others, less so (Sandoval and Gould, 1978, p. 500). No really long-term sites exist, however, that are diagnostic of the ultimate movement of sodium in spoils. The use of plant species that are tolerant to sodium-rich soils would not offer a complete solution since plant diversity would be limited. On most raw spoils there are few sources of nutrients and little biological activity that makes plant nutrients available. Nitrogen levels are also low. Adding fertilizers is usually a short-term solution, even though it may help plants become established. In some cases, the annual plant debris do not decompose, and therefore nutrients do not recycle into the soil. The addition of topsoil to the raw spoil would aid in the reestablishment of soil micro-organisms whose biological activity would return nitrogen to the spoils. Thus, the Office of Surface Mining's general requirement for topsoiling should help to ensure reestablishment of the nutrientrecycling that is necessary for revegetation and soil development (Cundell, 1977, p. 301-303).

\section{TOXIC SUBSTANCES}

Although the uptake and concentration of heavy metals or other potential toxicants by plants may be detrimental to the plants and consequently to the animals that feed on them, few confirmed examples of toxicity have been directly related to western coal mine spoils. Localized abnormal concentrations of toxic or plant 


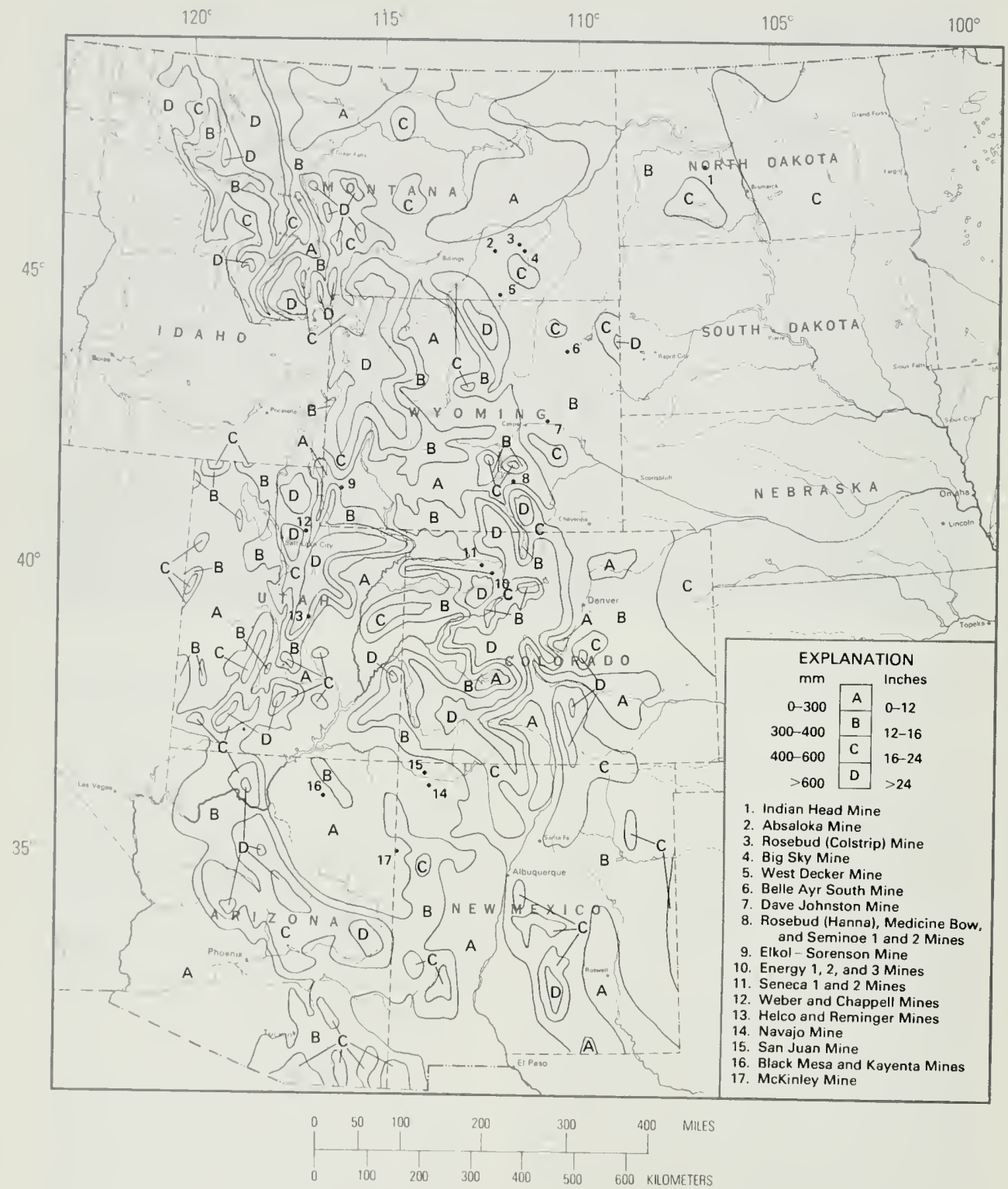

FiguaE 15.-Mean annual precipitation for the western coal region States based on period 1931-60. (Source: U.S. Department of Commerce, 1968, Climatic Atlas of the United States.) 


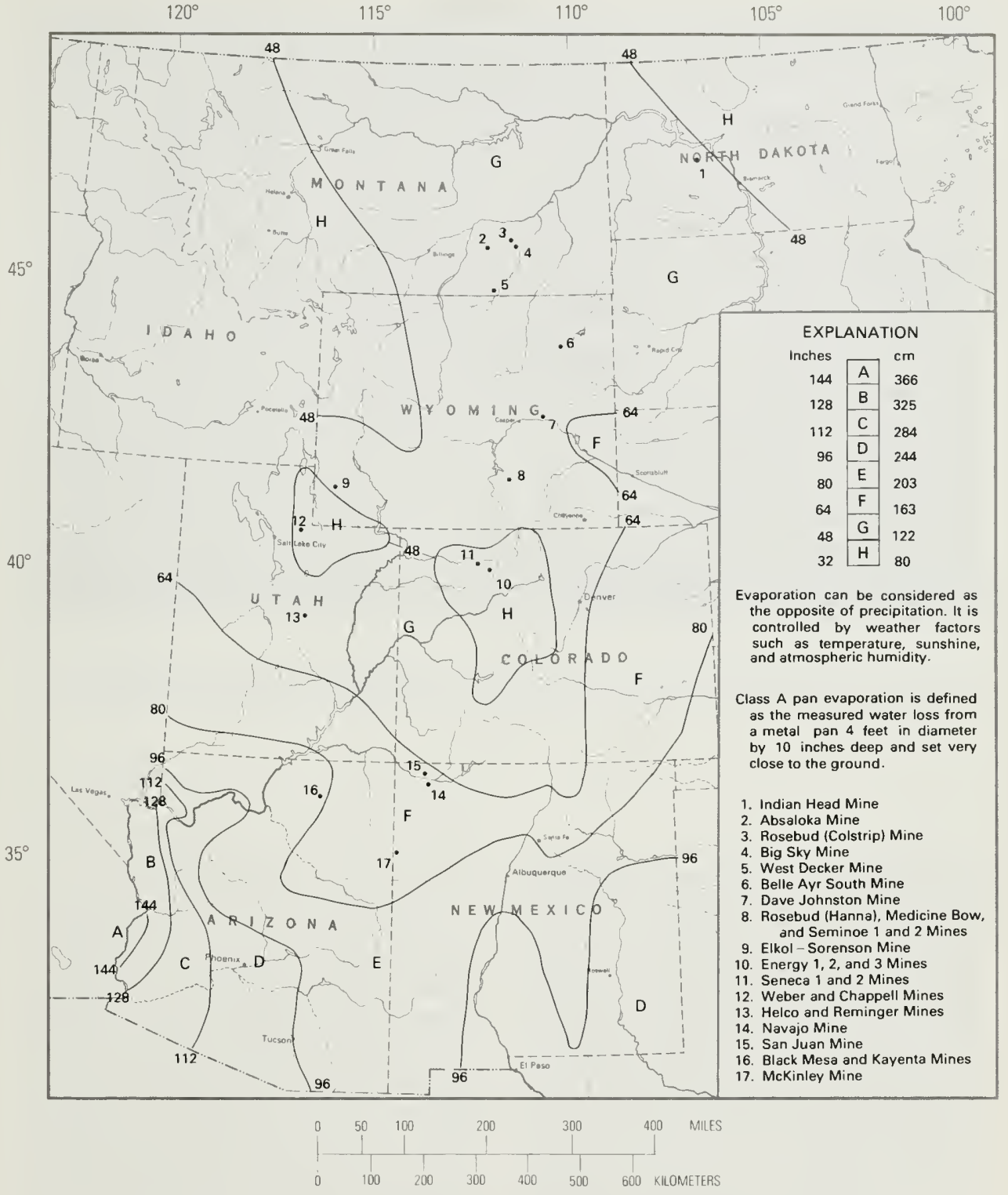

FIGURE 16.-Mean annual pan evaporation for the western coal region States based on period 1931-60. (Source: U.S. Geological Survey, 1970, National Atlas of the United States.) 


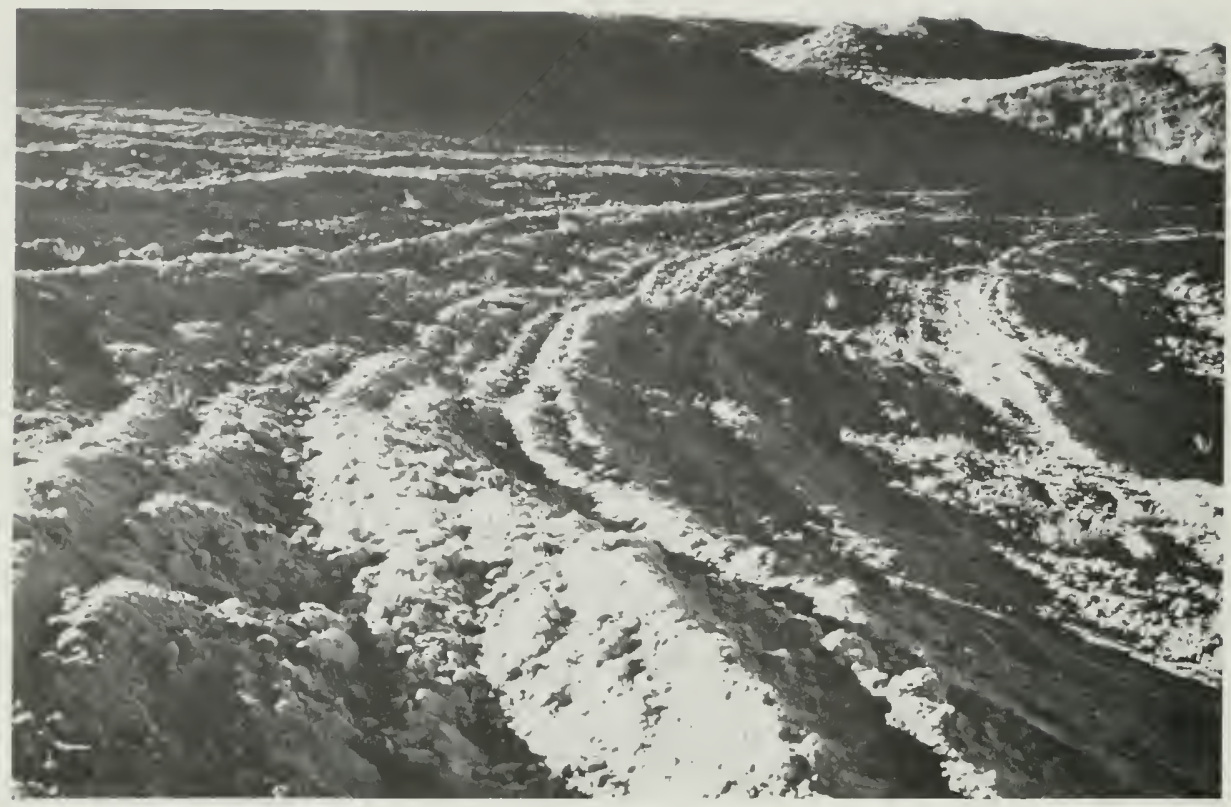

FIGURE 17.-Grooved, spray-mulched, and seeded slope at Seminoe 2 mine in Wyoming. This type of treatment is quite successful and conforms to the natural landscape. The grooving, done with a chisel plow, will become less evident with time. Grooving helps to conserve moisture, reduce wind erosion, and prevent widespread rilling caused by sheetwash. October 1978.

growth-inhibiting substances in overburden can be diluted by mixing materials in the excavation process. Where these substances have been identified in advance by test drilling, the affected overburden can be deliberately diluted by slightly modifying dragline practices. More extensive toxic layers can be immobilized by various burial methods (Dollhopf, 1979). Most potentially toxic metals are only marginally soluble under the highly alkaline conditions in western overburden and soil; therefore, they are not readily available for plant use. In the absence of significant copper under alkaline conditions, however, molybdenum can be taken up in excessive amounts by legumes. Boron can also form toxic compounds in plants grown on sodiumrich spoils.

\section{SOILS}

The replaced growing medium for revegetation is important in all stages of reclamation and is probably one of the most significant factors in long-term successful reclamation. The original soils are a product of the long-term interaction of the climate, parent material, landform, vegetation, and animal life that developed on and in them. Soils form a critical part of the ecological system; they contain a living system of their own. Their chemistry, texture, and thickness is adjusted to the land surface slope, and to elevation, temperature, and exposure. In theory, the replacement of the original soils with a reconstructed soil of similar characteristics would be a requirement for long-term successful restora- 


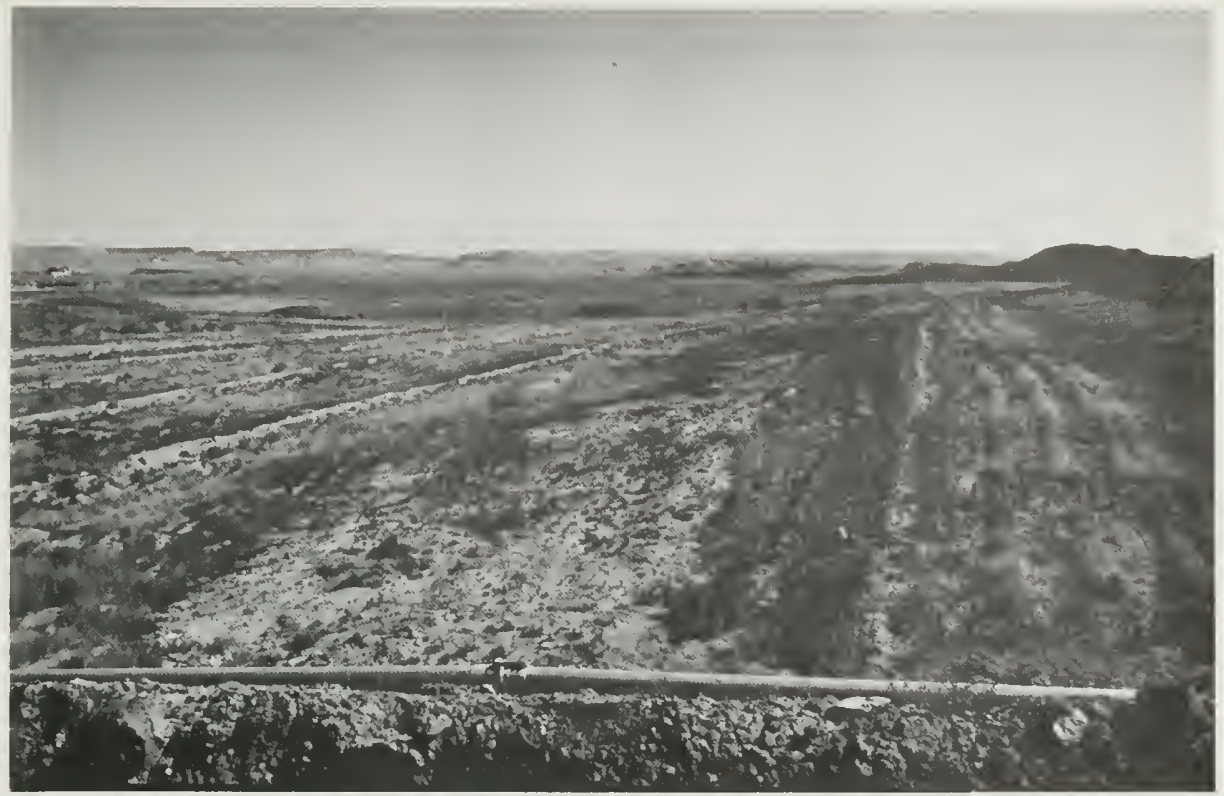

Figure 18. - Experimental water detention terraces at the San Juan mine in New Mexico. Only the lower parts of the terraces were seeded and irrigated. A section of the irrigation pipe is shown in the foreground. The terraces not only concentrate water but also reduce sheetwash erosion. July 1978.

tion. Complete restoration per se, however, is recognized as not being an achievable goal in reclamation because it is technically impractical. The enormous complexity of dealing with ecological systems can be illustrated by examining a soils map of a mining property (fig. 25). This map shows the large number of different soils that exist within a relatively small area.

The use of original soil materials on newly shaped mined lands, even if mixed during the salvage process, generally has five major effects on the revegetation potential, in comparison to raw spoil materials. Original soil materials (1) provide a seedbed with more desirable physical properties; (2) introduce more plant-available nutrients; (3) act as a better medium to absorb and hold moisture; (4) provide a source of native seeds and native plants; and (5) provide bacteria, algae, fungi, and other forms of soil flora and fauna theoretically capable of reestablishing the soil-forming process.

\section{TOPSOILING}

The reuse of the original soils as all or part of the new growing medium in reclamation is called "topsoiling." The advantages of topsoiling for the reestablishment of any form of vegetation are now well recognized. Even thin topsoil layers have proven advantageous where the spoil is somewhat toxic. The optimal plant-growth relationship to topsoil thickness under economic and supply constraints is being studied at many surface coal mines (Deveraux, 1978, p. 200; and Pole and others, 1979) (figs. 26 and 27). Topsoil seems to be more important to long-term success than various cultivational techniques which are useful for improving the physical characteristics and water-holding capacity of the growing medium. Spoil materials alone, however, may be able to support a vegetative cover as productive as that present before mining under the 


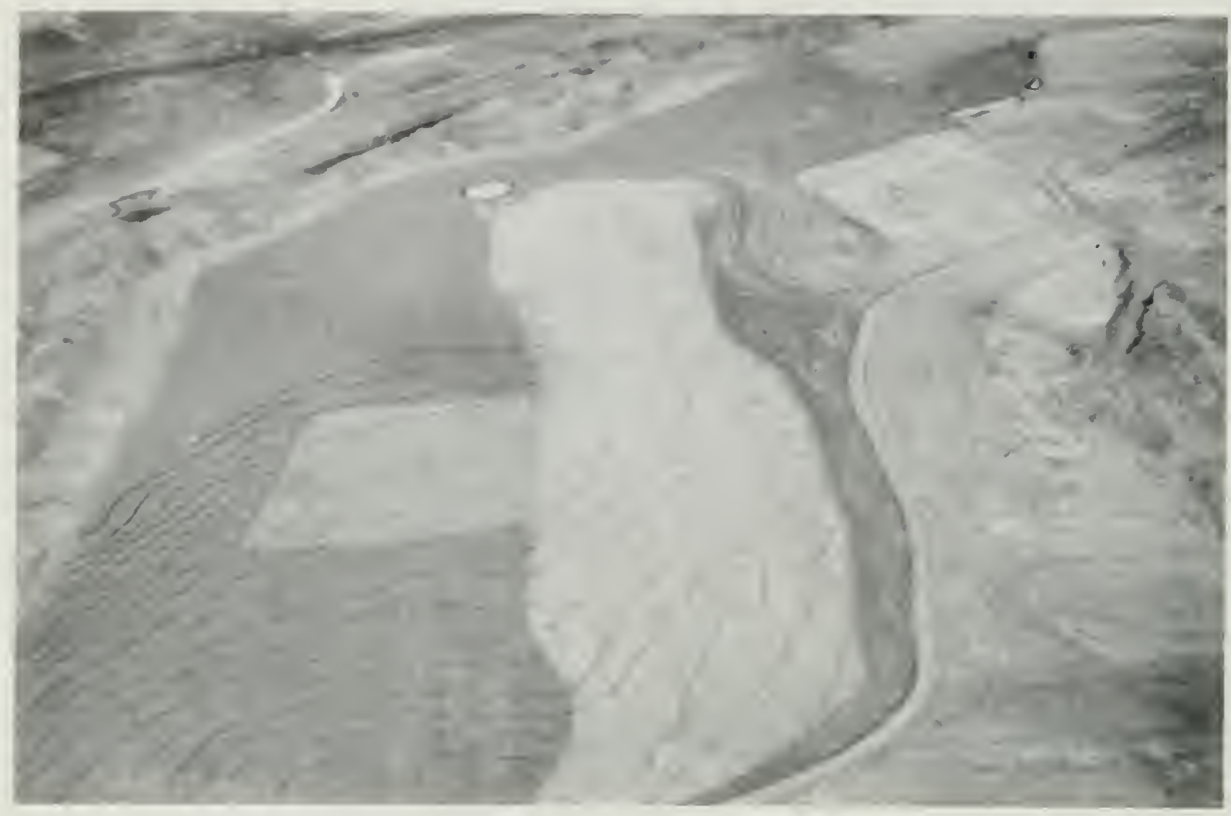

FIGURE 19.-Aerial photograph showing mining and reclamation sequence at Energy 1 mine in Colorado. Contour furrows (erosion control trenches and berms) parallel to topographic contours are cut in freshly graded and reshaped spoil (center) next to new spoil (right side) and in second-season grasses (left). Mining, marked by spoil piles, is proceeding in the left and upper areas. Some small impoundments are produced in the smoothing operation (center and upper right). November 1978.

right conditions of slope, climate, and composition (Schumacher and others, 1977, p. 19).

Interpretations of what comprises a topsoil suitable for reclamation have varied widely in the past because the term does not have a clear scientific definition even in soil science use. Generally topsoil is considered an "enriching layer" for plant development. In coal-mining usage, topsoil has included (1) suitable overburden alone, usually that material whose chemical or physical properties are more favorable than the existing soils; (2) mixes of overburden (fig. 28) and original soil materials, at times required when original soil is thin, rocky, or otherwise poorly constituted (fig. 29); (3) only original soil materials, consisting of a mixture of the upper layers, the final thickness of which depends upon soil testing and the skill of the scraper operator; and (4) the uppermost distinct soil development layer generally referred to as the "A" horizon. Office of Surface Mining regula- tions generally require that the top 6 inches of the old surface be stockpiled and added to the top of the new surface (U.S. Office of Surface Mining, 1979, p. 15397); these regulations, however, permit mixtures of soil and overburden or substitutions of overburden under certain conditions.

\section{BIOLOGICAL FACTORS}

Biological factors affecting revegetation exist both above and below the surface. The importance of subsurface biological components such as bacteria, fungi, and arthropods, was briefly discussed in the preceding sections. These components are believed to be necessary in establishing a self-sustaining vegetative community where large areas are disturbed by mining operations (Cundell, 1977, p. 299). The Forest Service (Lindsey and others, 1977; Williams and 


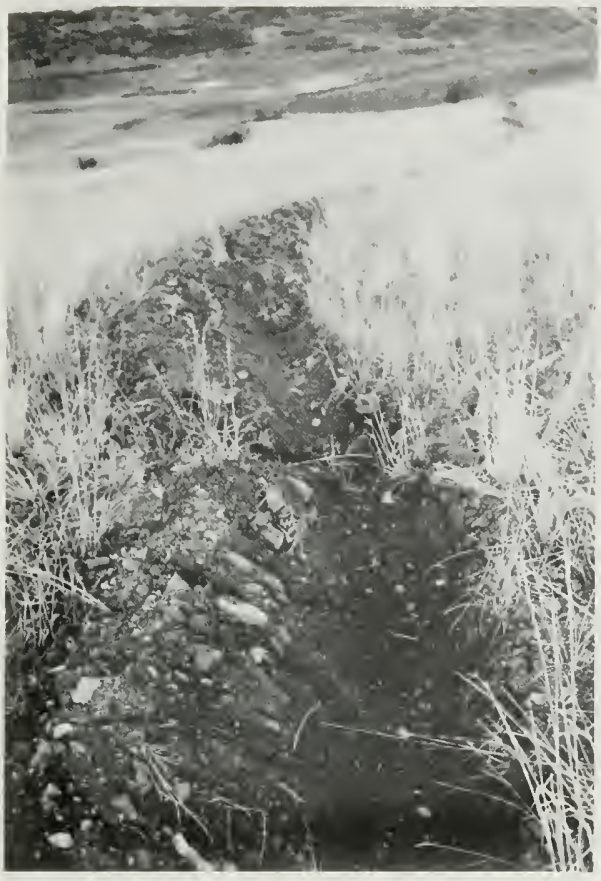

FIGURE 20.-C!oseup of a contour furrow at Energy 1 mine in Colorado. The furrow is closed off at 24-foot intervals to prevent water from creating a larger watercourse or channel. The furrow, including ditch and castings, averages 31 inches wide and about 16 inches deep, and it follows the hillside contour. Furrows have been successfully used to control erosion in the area. November 1978.

Aldon, 1976; Aldon, 1975 and 1978) has investigated the role of beneficial root fungi (mycorrhizae) in establishing certain woody plants on western mining spoils. Such mycorrhizae, which could be inoculated directly into the new soil medium or via newly planted stock, aid considerably in the plants' capability to absorb soil nutrients. Seedlings inoculated with mycorrhizae seem to be more drought resistant, to become more quickly established, and to have higher survival rates on poorer sites. Despite the apparent value of inoculation for many plant species, the Forest Service Shrub Laboratory does not routinely inoculate its containerized plants (Robert Ferguson, U.S. Forest Service, oral communication, 1979) because fungal inoculation has not reached a practical production stage (Kuja, oral communication, 1979; Zak, 1975). Some reclamation plant species can flourish without the deliberate in. troduction of mycorrhizae. At the densest stand of seeded fourwing saltbush seen on raw mine spoils, no mycorrhizal inoculation was used (Grogan, oral communication, 1979) (fig. 30). This stand, however, had been heavily irrigated. Apparently the fungal spores can be carried by wind from adjacent areas or can be introduced through topsoiling when it is used. In general, the grass species do not rely as much on mycorrhizal relationships (Cable, 1977; Kuja, oral communication, 1979) as other species and, hence, grasses have proven to be the most adaptable plant family for reclamation.

Work is also being done to explore the potential role of termites in breaking down raw organic material such as straw mulch, and in improving the physical and chemical charactristics of raw spoil (Ettershank and others, 1978).

The greatest biological detriment to reclamation is overgrazing by domestic livestock (fig. 31). Permitting cattle and sheep into planted areas before they are fully established has totally destroyed some plantings (fig. 32) and has greatly weakened others (Schumacher and others, 1977, p. 13). Most coal-mining companies now do not permit grazing on land they are trying to reclaim during the period of mining (table 1).

Reclamation success is also affected by wildlife. The initial effect of wildlife on revegetation is loss of seeds and the overbrowsing of young woody plants, particularly where these plants are sparse. Most mine demonstration and test plots that are not fenced are being heavily browsed by wildlife. Berg has indicated (1975, p. 82-83) that where deer and elk are plentiful, revegetation of some species could be difficult because of overbrowsing. Seeded or planted woody species can be protected from overbrowsing if they are surrounded by herbaceous vegetation.

In some areas, heavy degradation by seed- and seedling-eating rodents, rabbits, and birds has retarded reclamation efforts until artificial habitats and perches for predatory species were added. The role and linkage of animal, insect, and bird ecological systems - their food chains, their pollination, and seed dispersion roleshave been poorly addressed in active reclama- 


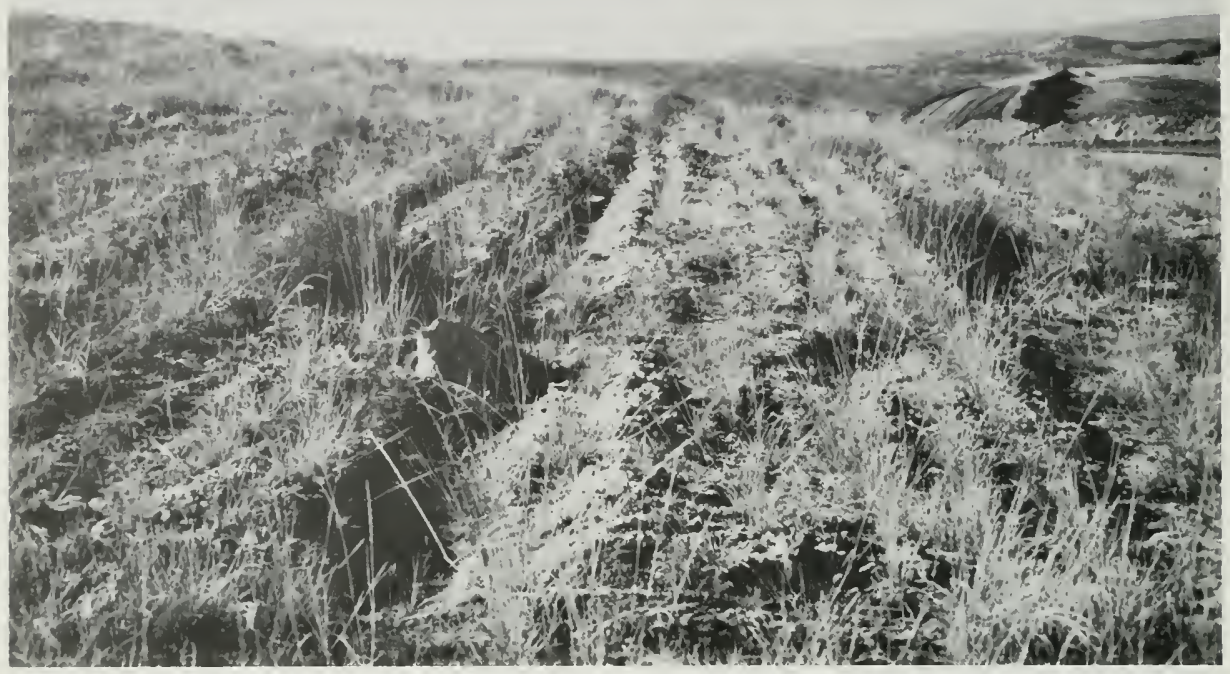

FIGURE 21.-Rough, ditched, and grooved reclaimed area adjacent to recently smoothed spoils and spoil piles (right) at the McKinley mine in New Mexico. The rough surface texture is used to catch and hold precipitation. The 2-year stand of grass shown here seems to indicate good potential for successful revegetation in this area of low annual precipitation ( 9.0 to 12.5 inches). November 1978

tion research to date. Such relationships have been only addressed in a limited way through efforts to recreate habitats for specific kinds of wildlife. In general, these efforts have been directed at establishing habits for certain game species, particularly big game species, and consequently plants known to be palatable to game have been planted.

\section{VEGETATION}

Vegetation, as well as being the final objective, is also an integral element of the reclamation process. Quick-growing, short-lived species are planted for temporary cover on stockpiled material and occasionally to provide shade and mulch for slower growing, more permanent species. Weedy annuals, however, especially Russian-thistle, tend to dominate newly disturbed ground and newly planted areas. Russian- thistle, an annual plant, seems ubiquitous for first-year growth on many western coal mined lands whether the lands are abandoned or planted (fig. 33). The growth of Russian-thistle can be misleading to the casual observer with respect to the effectiveness of reclamation, however, because its dense green cover, seen in summer, dies and is blown away in winter. As described earlier, annuals can form a large percentage of the plant community for some time on abandoned mined land. For a permanent allseason cover, however, perennial plants are required.

The primary goal of early reclamation attempts was to stabilize spoils to reduce erosion. Perennial grasses planted at that time were mostly nonnative (exotic) species that were tough and adaptable but not necessarily useful for grazing or conducive to the establishment of a diverse native plant community. Later the grass species being planted were upgraded to 


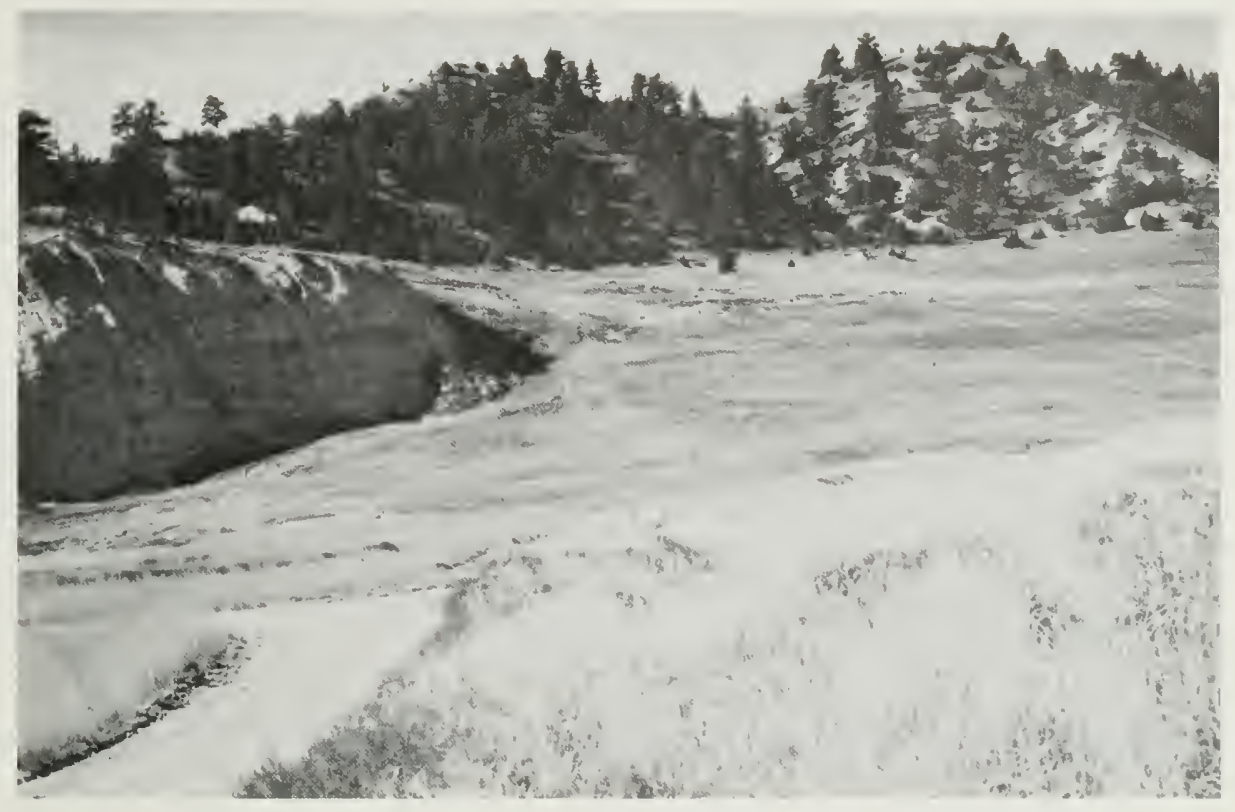

FIGURE 22. - A graded highwall at the Big Sky mine in Montana. Little, if any, topsoil was added to the sodic spoil in the background. Fifteen to 20 inches of topsoil was added to the right foreground area which was seeded in 1975 and reseeded in 1976. Vegetation consists mostly of annuals and some wheatgrasses. October 1978.

include more productive strains and more palatable species and included some native species (Cable, 1977). Legumes (alfalfa, white clover, and yellow clover) were also included. The broadened objective then became both erosion control and establishment of grazing areas for livestock on rangelands.

More recently, seedlings or seeds of woody browse species are being planted (fig. 15). Introduction of fourwing saltbush by seeding has been particularly successful on saline sites (fig. 30). In general, the two objectives of ground cover-erosion control and biological productivity-have stayed much the same; the additional goal of a "diverse, effective, and permanent vegetative cover" has come under OSM regula. tion. OSM's "measures of success" are made by comparing the ground cover and above-ground productivity with that of adjacent undisturbed reference areas (U.S. Office of Surface Mining, 1979, p. 15413-15414).

The plants native to the semiarid western coal-mining areas are the end products of thousands of years of selective development and adaptation. At any one mine, a variety of vegetative communities will reflect differences in soils, slope, moisture, and use. These vegetative com. munities also have associated with them specific wildlife species. Recreating the original vegetative communities could be virtually impossible, given the changes in landforms and soils. The goal of the regulatory authorities is, however, to reestablish native plant communities to the greatest degree possible.

Considerable work has been done and is being done in the selection of better plants for use in reclamation. Many researchers have reported on the variables, made recommendations, and identified problems in planting (Monsen and Plummer, 1978), but limited research has been done on the selection and improvement of native plant species to support the needs of local wildlife. Many of the grasses now used in reclamation planting are nonnative cultivars 


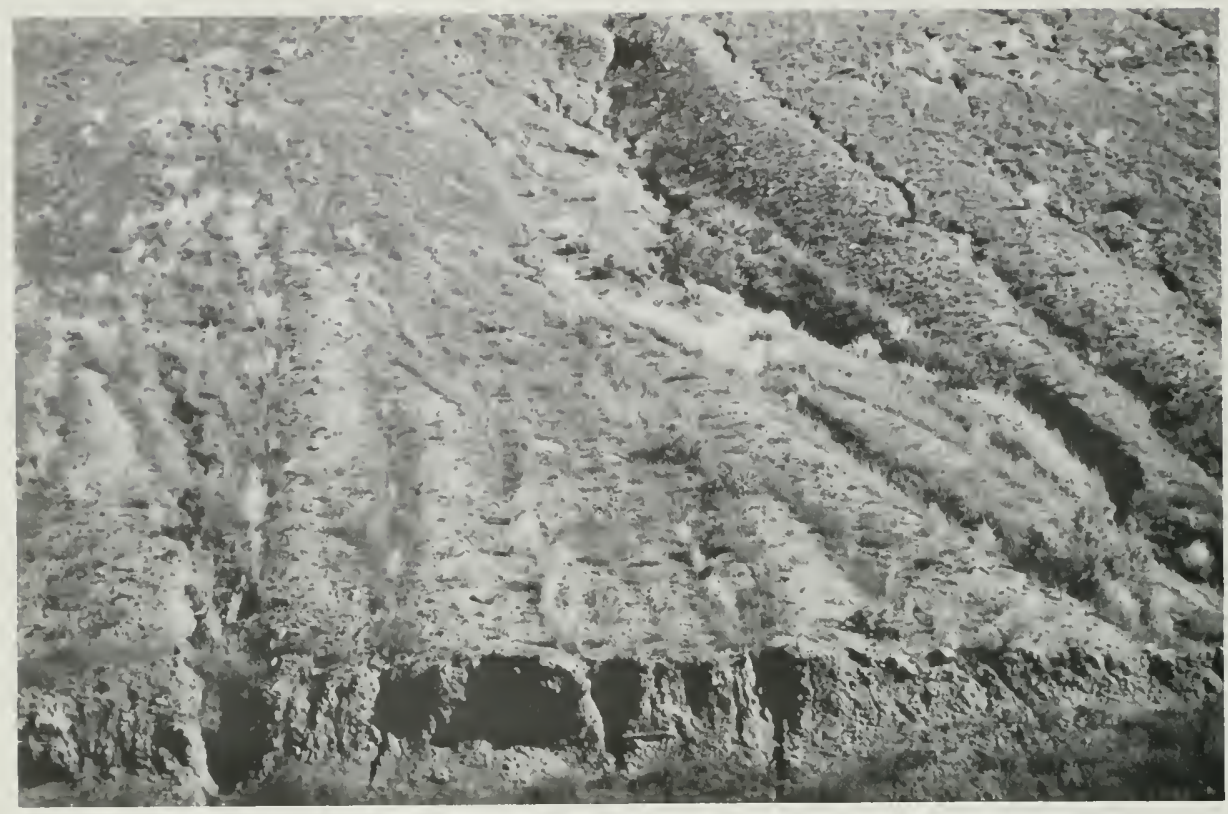

FIGURE 23. - Eroded steep slope and natural revegetation of an old spoil pile along a haulroad of the Seminoe mines in Wyoming. Reclamation practices depicted here generally conform to the 1969 Wyoming Open Cut Act, which predates the stricter 1973 Wyoming Environmental Quality Act. Volunteer ricegrass and shrubs grow in moisture-bearing gullys and rills. The base of this slope has been undercut by the drainage ditch of the haulroad and its instability and rate of erosion have been increased. October 1978 .

selected for their adaptability over wide areas and under adverse conditions. In the Western States, many of these grasses have been selected by the Soil Conservation Service's Plant Materials Centers (Power, 1978, p. 530-531). The U.S. Forest Service has established an arid land shrub selection and experimental breeding program (Monsen and Plummer, 1978, p. 172-173) in addition to its long standing research on both native and nonnative grasses for coal mine reclamation (Bjugstad, 1978; B.Z. Richardson, U.S. Forest Service, oral communication, 1980).

The Plant Materials Centers make the selections for testing on the basis of each plant's expected widespread adaptability to the type of site, its persistence after establishment, and its intended use, for example, wildlife or livestock forage. Their evaluation technique is based on monocultures of the tested plant; consequently, the behavior of the plant when in competition with other species is unknown (Power, 1978, p. 530-531). Although nonnative (exotic) plant selections were found to produce significantly more range forage (but providing less soil cover) than the native plants (Packer and others, 1981), the nonnative species have a tendency to be displaced by native plants over time in all types of western reclamation (P.E. Packer, U.S. Forest Service, oral communication, 1979; Gillin, 1980, p. 66).

The Plant Materials Centers disseminate plant selections through cooperating Federal and State organizations to see that the plants get into the commercial seed markets. The centers also prepare lists of the seed sources for distribution at the local level by the Soil Conservation Service. The Centers have collectively published a summary handbook on plants suitable for reclamation work in arid and semiarid regions (Thornburg, 1980). Some 200 plants, 


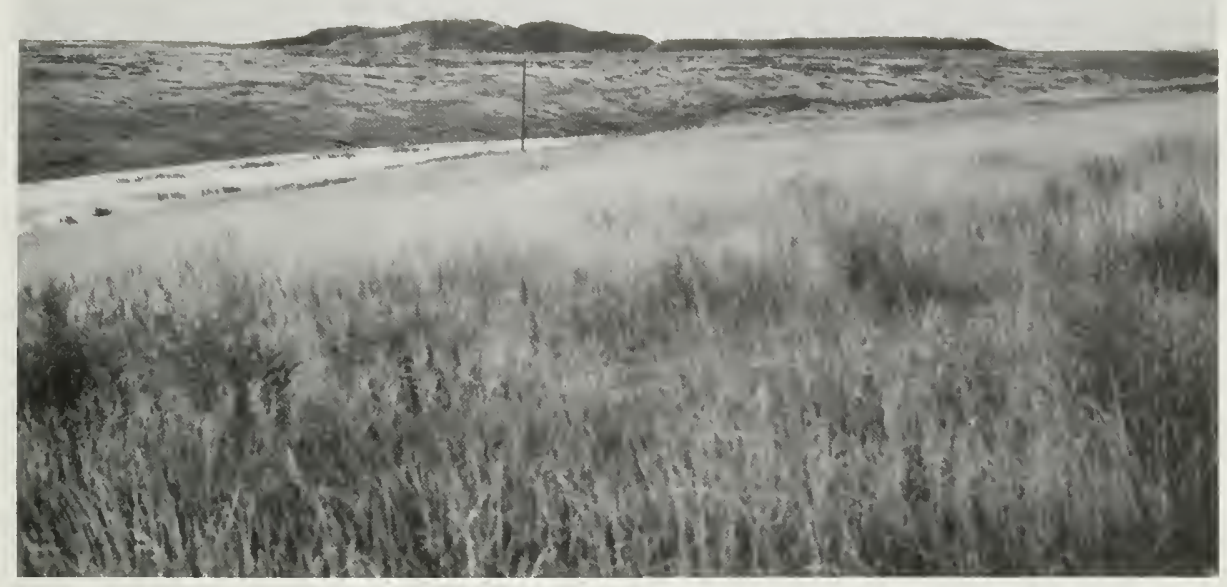

FIGURE 24.-Contrasting vegetation at the Indian Head mine in North Dakota. The sloping spoil in the background has a high sodium content. It is nearly devoid of vegetation and contrasts sharply with low-sodium spoil covered by third-season grasses in the foreground. The pond (center left), locally invaded by reeds, makes an excellent habitat for watertowl. Old spoil piles in the distant background were regraded. As a result, spoils having high levels of sodium were exposed, and the regrading actually resulted in a worse situation. October 1978.

about 50 percent of them native species, have been selected. Attention is now being concentrated on native plants, which comprise 70 to 75 percent of most recent selections. In addition, forbs (nongrass herbaceous plants) and shrubs are now being selected for testing (Robert McLachlen, U.S. Soil Conservation Service, oral communication, 1979).

The U.S. Forest Service Research Shrub Science Laboratory has done extensive work on transplanting containerized native plants, shrubs, and trees on disturbed lands (Frischknecht, 1978; Cable, 1977; Monsen and Plummer, 1978). An extensive list of plants, including native grasses, evaluated by the Forest Service for adaptability to various disturbed site conditions, climatic situations, methods of establishment, and for rates of natural spread has been published (Plummer, 1977, table 31.1, p. 323-337).
Seed has been sown in a variety of ways in western reclamation-by aerial and ground broadcasting, seed drills, as well as hydroseeding with mulch. According to Packer and Aldon (1978, p. 435-438), there are preferred times and depths at which to sow different kinds of seed and plants for the best results. One of the problems facing reclamation personnel has been locating adequate sources of seed or planting materials of many native and naturalized species. Even when located, the seed may be very expensive and available in only limited quantities. Seed of Galleta grass (Hilaria jamesii), a species adapted to alkaline soils, was reported to cost more than $\$ 50$ a pound in 1977 . The seeds of this and some other species must ripen over a period of time, and they are difficult to collect. Other species that grow as widely dispersed individual plants in the wild, or that produce seed only at long intervals, will always be 


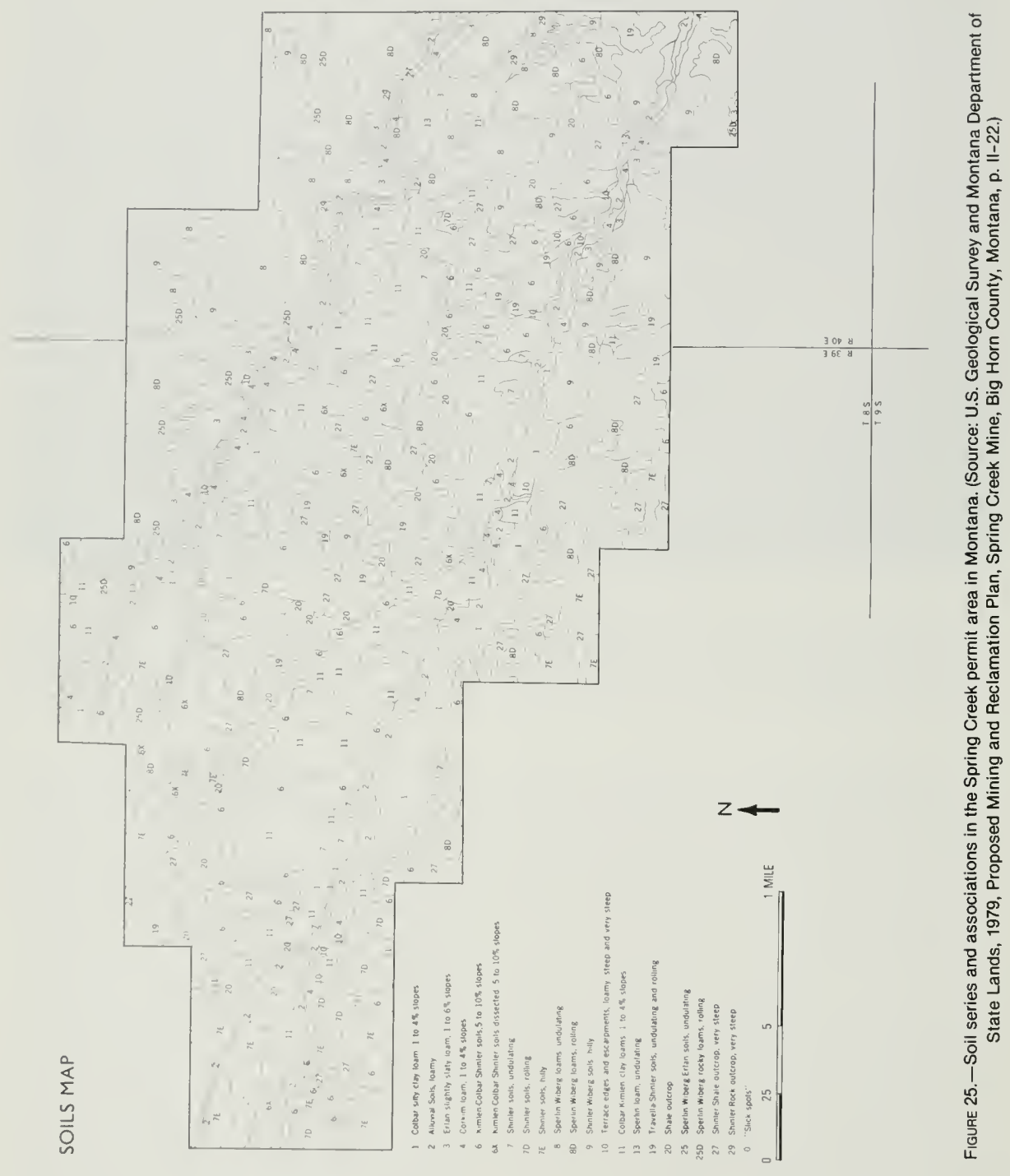




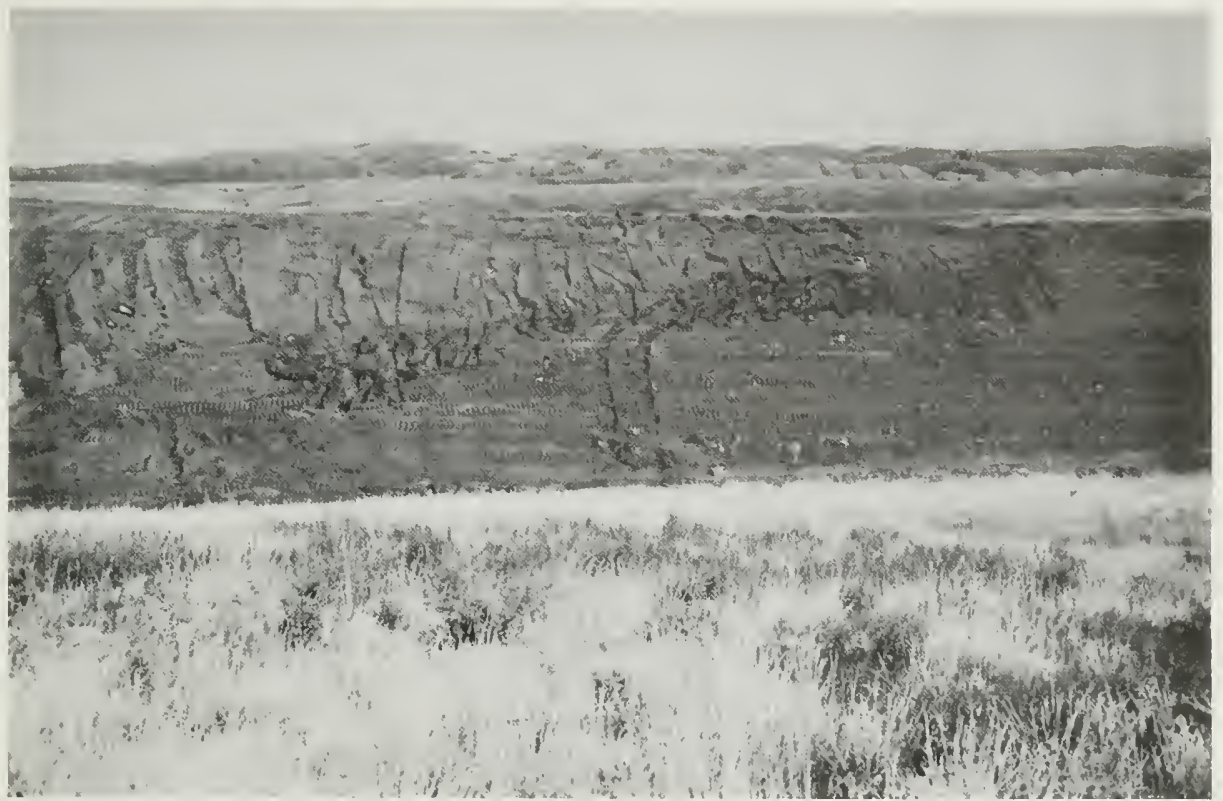

Figure 26. - Topsoil being spread on regraded spoil at Seminoe 2 mine in Wyoming. Topsoil is dumped from trucks along the ridge and spread in two directions by a bulldozer. Grass and forbs in the foreground represent several seasons of growth on similarly reclaimed land. Long, steep slopes such as this one do not always fit into the natural landscape and, in places, have been eroded more readily than the gentle slopes in the foreground. Mining, marked by spoil piles in the background (right side), is proceeding away from the observer. October 1978.

hard to collect and will be expensive. Although the number of seed collectors and growers of transplants and container plants has increased (Crofts and McKell, 1977), their locations are not always known to mining management personnel. Many seed firms do not routinely stock the desired seed but collect or grow it only on order. Locating plants or seeds that are genetically adapted to the growing site presents an additional complication because seed from a warm or dry site may not perform as well in a cold or moist site (Thornburg and Fuchs, 1978, p. 413). Monsen and Plummer (1978, p. 173) believe that expanded efforts are needed to produce enough seed and transplanting materials to meet the large-scale reclamation needs of western coal development.

If a mining company wants to reproduce a specific plant community, the company may find that seed or planting stock for many species is unavailable commercially. Such species can be reintroduced from seed or root stock pieces in the topsoil or may eventually be blown onto the site from the outside (Wagner and others, 1978), but odds favor a long time to develop a plant community by these means. In greenhouse studies, Beauchamp and others (1975) found substantial amounts of viable seed from succes. sional type plants in the top 2 inches of Wyoming topsoils; comparatively little seed, however, was present from the permanent plant species that dominated the sites. Introduction of aggressively competitive, mostly annual successtional species has been considered a negative aspect of topsoiling (Hodder, 1978, p. 152; Frischknecht and Ferguson, 1979, p. 24).

The possibility of collecting seed or transplants of specific species from adjacent un- 


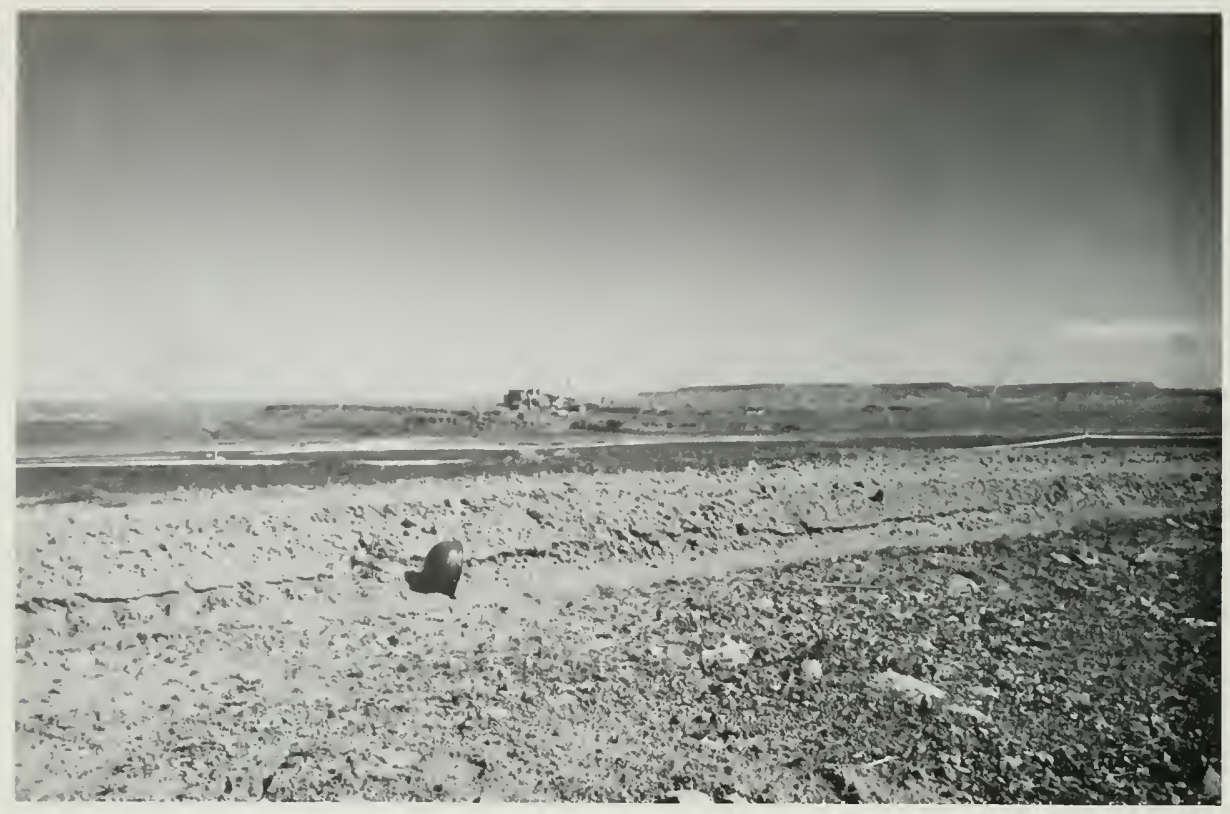

FIGURE 27.-Sprinkler irrigation on topsoiled, graded spoils at the San Juan mine in New Mexico. One foot of topsoil placed on the spoil is seeded and then irrigated by the sprinkler system in the background. About 6 to 8 inches of water is supplied to the seeded spoil during the first two growing seasons. This thickness of topsoil was found adequate through extensive prior tests. The San Juan generating station is in the background. August 1978.

mined lands has been considered by mining companies (Chase Caldwell, Navajo mine, oral communication, 1978). Transplanting patches of native sod or individual plants, even if the prin. cipal plants die (fig. 34), provides a source of a variety of native plants as well as soil biota.

At the time of this investigation, the new Federal reclamation regulations (U.S. Office of Surface Mining, 1979) were just starting to be enforced, and exactly how they would be interpreted in the field was not fully known. Presumably, most reclamation approaches would be decided on a case-by-case basis. It is the intent of the regulations in paragraphs 816.111 and 816.112 to require a diverse vegetation with approximately the same level of seasonal utilitarian plant life forms that previously existed. Replanting with native species is preferred, but introduced species will be approved on a limited basis. The plant cover should be "a mixture of species of equal or superior utility [to the natural vegetation] for the approved postmining land uses..." The OSM standards for success, however (par. 816.116), do not mention diversity or native species per se, but only the percentage of ground cover and its productivity. Although these regulations address postmining uses for grazing (on rangelands) and wildlife management individually, people applying the regulations have tended to aim for a combination of both, as indicated by the premining multipurpose uses planned for most of the mines sites examined.

Although neither diversity nor the proportions of native plant species have been specified quantitatively, OSM interpreted paragraph 816.111 (a), "a diverse, effective, and permanent vegetative cover of the same seasonal variety native to the area... or species that support the approved postmining land uses," to mean that a 


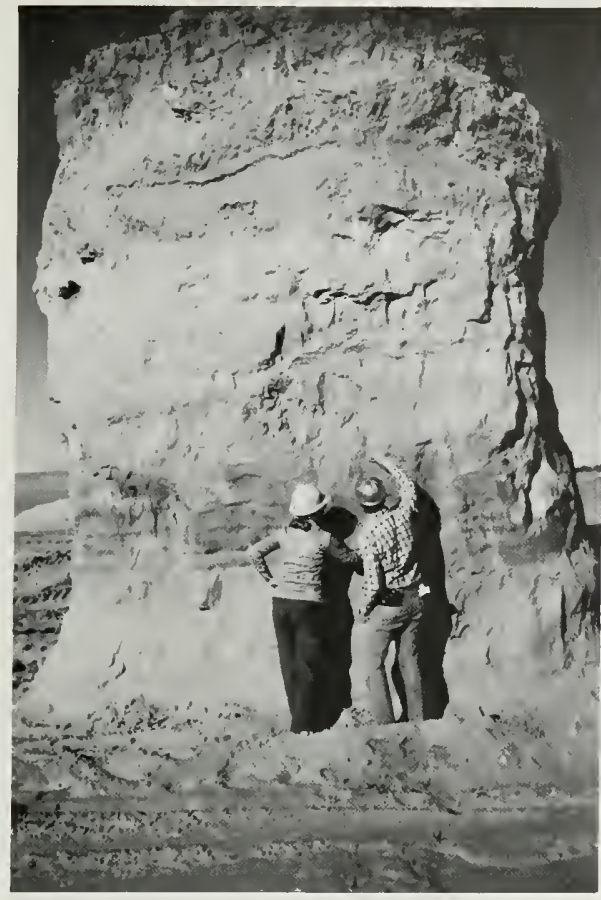

Figure 28.-Soil column used for State certification of recla. mation topsoiling at the Black Mesa mine in Arizona. The darker areas in the soil column are carbonaceous fossil soil zones. All of this predominantly sandy soil zone, represented by the column, is used for topsoiling. In this region of generally poor soils, only the upper foot is considered as topsoil. November 1978.

significant proportion of the vegetation should be native species and have a diversity potential approaching that of the premined land. One of the specific conditions under which introduced species can be substituted for native species is when "the [introduced] species are compatible with the plant and animal species of the region" (par. 816.112(c); this paragraph exemplifies the need for greater diversity. Because of the limited soil moisture resources of the western coal region, however, it is difficult to assume that a successfully introduced competitive plant would be compatible with all existing species. The premining diversities shown in table 1 probably represent only the more numerous species, except for the 163 species identified at the McKinley mine in a research study by Wagner and others (1978, p. 69). Power (1978, p. 531) has shown that many rangelands contain over 40 plant species. Wali and Freeman (1973, p. 31-39), in examining seven North Dakota coal mining areas, reported that the adjacent unmined lands contained from 11 to 23 plant species. Whatever the true values may be, past reclamation efforts evidently have not approached premining diversities in species number, and even less in plantlife forms and proportion of native species.

\section{OPERATIONAL DIFFERENCES}

The mines examined in this study were visited by the team in about 10 days, although various members of the team had visited specific mines at other times. Because the time spent at each mine was short, the following observations on differences in reclamation operations are qualitative only.

Many of the mines visited had been active for 15 years or more, but others were comparatively new (1 to 5 years old). All, however, operated under changing reclamation directives from State and Federal agencies; these changing regulations and guidelines produced differences in ways of shaping the land, handling drainage, and encouraging revegetation. A wide variation also existed in corporate compliance with and interpretation of reclamation laws. At some old mines, the spoils remained abandoned; at others, corrections were made voluntarily (fig. 35); and at some mines more problems were created by reclamation attempts (fig. 24). Many mining companies had foreseen impending changes in regulations and had reacted accordingly to ensure the continuity of the mining. Some companies were even ahead of the regulatory requirements in applying new and advanced reclamation techniques. Many companies had cooperated in large-scale research projects and test plots supervised by Government and State agencies and by universities. A few companies had experimented on their own with reclamation practices.

Part of the difficulty of operating under regulations is the way in which they are interpreted and enforced. Within a State or at adjacent mines, official guidance had sometimes been different 


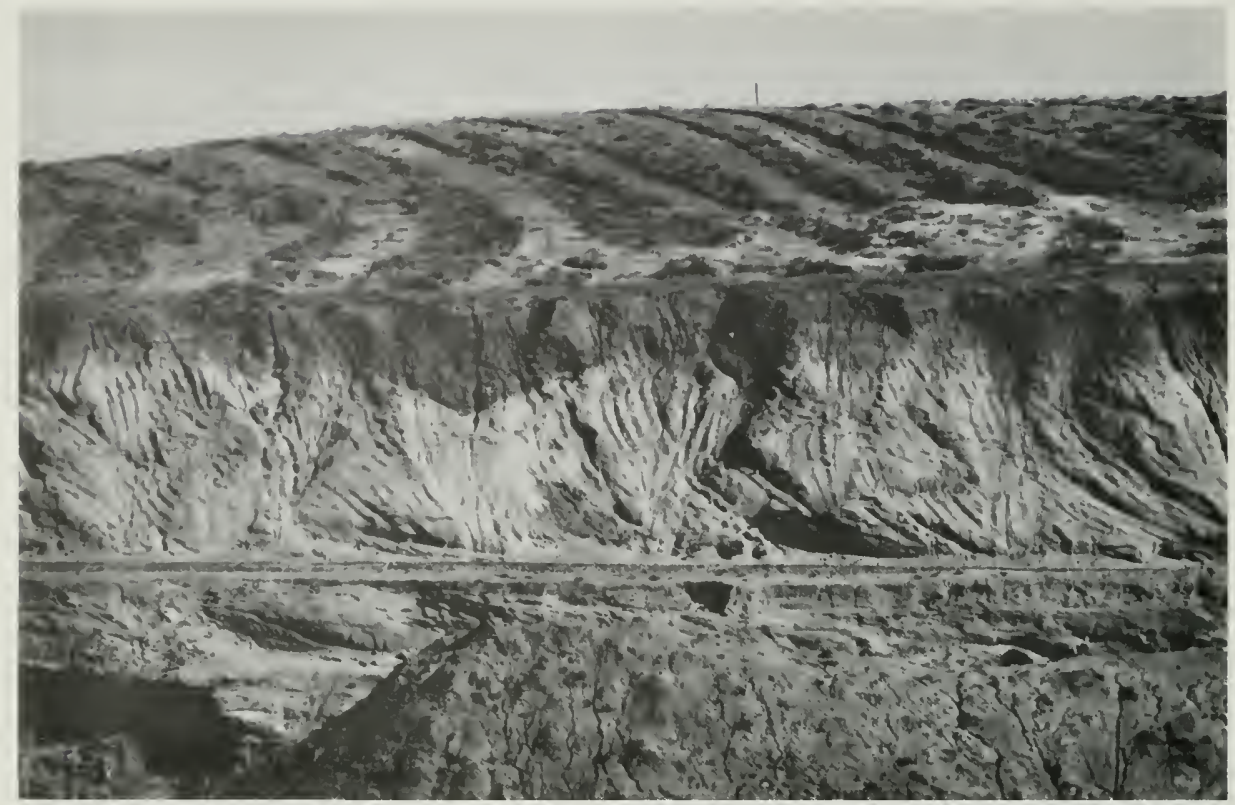

Figure 29. - Topsoil borrow strips (top) at the Dave Johnston mine in Wyoming. The soil borrow strips, about 10 feet wide, were reseeded with western and crested wheatgrasses. Sagebrush occupies the undisturbed strips. In the center, the first bench of overburden has been removed and exposes a dark soil zone of irregular thickness that covers white silty sandstone. In this instance, only the upper 6 to 10 inches were used for topsoiling. Eroded spoil is in the near foreground, opposite the box cut. Mining is proceeding toward the soil strips. October 1978.

under the same regulations. Turnover in reclamation staffs at some mines lead to a loss of continuity in activities and, in a few cases, negated the value of research plots. The appointment of a new inspector, for example, changed guidance at an individual mine in one reported instance.

At most of the mines visited, the reclamation staff was separate from and independent of the mining staff. Differences as to where mining ceases and to where reclamation activities begin were only evident when assessing the cost of reclamation. In all cases, both groups functioned as cooperating teams.

Reclamation success probably will be strongly influenced by the amount of corporate support given to the reclamation effort in terms of priority, staff, and technical support. Some reclamation staffs have done innovative experimental reclamation work. Time is needed to evaluate the success of these experiments, but early results indicate that some of this innovative work is of value (fig. 36). Most work has involved creating wildlife cover (figs. 37 and 38 ) and manipulating the surface to prevent erosion and conserve moisture (fig. 18). At some mines, public service clubs and scout groups have volunteered to help plant small trees and shrubs supplied by the mining companies.

Although the ingenuity of individual reclamation efforts was obvious, an adequate exchange of technical information and ideas was lacking. For instance, one mine was able to include an unusually wide diversity of species in its planting program because the reclamation manager was familiar with many sources of plants and seeds from a published compilation of sources by Crofts and McKell (1977); managers at some other mines, however, were unaware of this publication. Although reclamation specialists in some of the mining districts met professionally, the flow of information seemed to be restricted. 


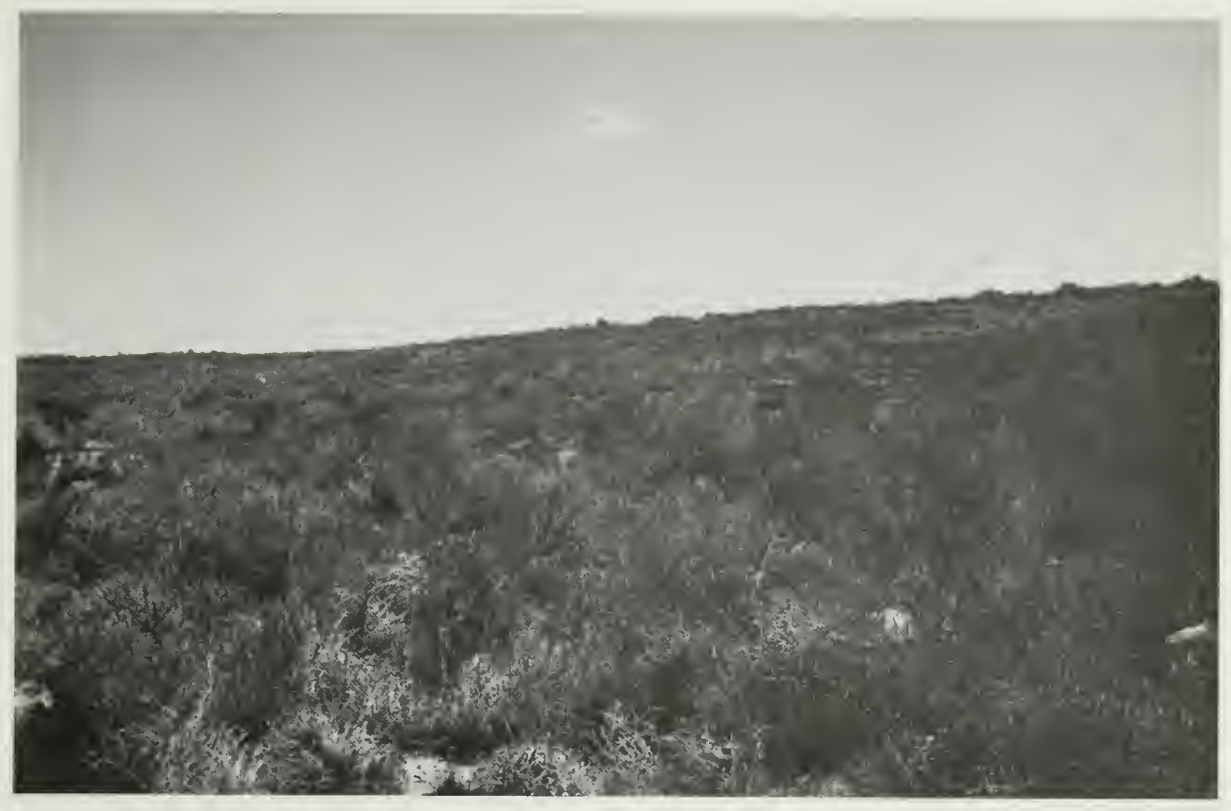

FIGURE 30.-Densely revegetated slope of fourwing saltbush and some grasses on sandy saline spoils that were seeded in 1975 and irrigated for two growing seasons at the Navajo mine in New Mexico. The saltbush was dense during irrigation, but it is now beginning to thin. July 1978.

The diffuseness of reclamation literature was assessed by Scott (1978, p.311-312), who called for an international journal on reclamation research. To solve the general problem of inadequate information exchange, workers from several Federal agencies met at an August 1979 coordination workshop on western mining reclamation and advised each other of the status of reclamation handbooks published, in progress, or needed. Between 1980 and 1982, the U.S. Forest Service, the Soil Conservation Service, and the Bureau of Land Management were to prepare reclamation handbooks for use in western coal mine reclamation work.

\section{ESTIMATES OF RECLAMATION EFFECTIVENESS}

Even with the broader perspectives gained from examining reclamation results at many western coal mines, this study cannot provide quantitative answers to the questions posed in the introduction of this report. The reconnaissance did, however, provide insight into the means for evaluating the issues and a sounder basis for evaluating potential reclamation on a case-by-case basis. A number of papers have predicted successful reclamation results, but none have dealt fully with native plant communities (National Academy of Sciences, 1974, p. 2; Frischknecht, 1978, p. 3; Hodder, 1978, p. 149, 154; Packer and others, 1981). Similar statements about reclamation success have appeared in environmental impact statements prepared by the U.S. Geological Survey and the Bureau of Land Management for proposed surface coal mines. A few reports take a more cautionary approach (Curry, 1975). Wali and Kollman (1977, p. 112), in proposing an ecological perspective (and a new term, "mining ecology"), are generally optimistic regarding the future potentials for returning mined lands to their original productivity levels but challenge statements that long-term productivity of reclaimed lands can eventually exceed that of the premined 


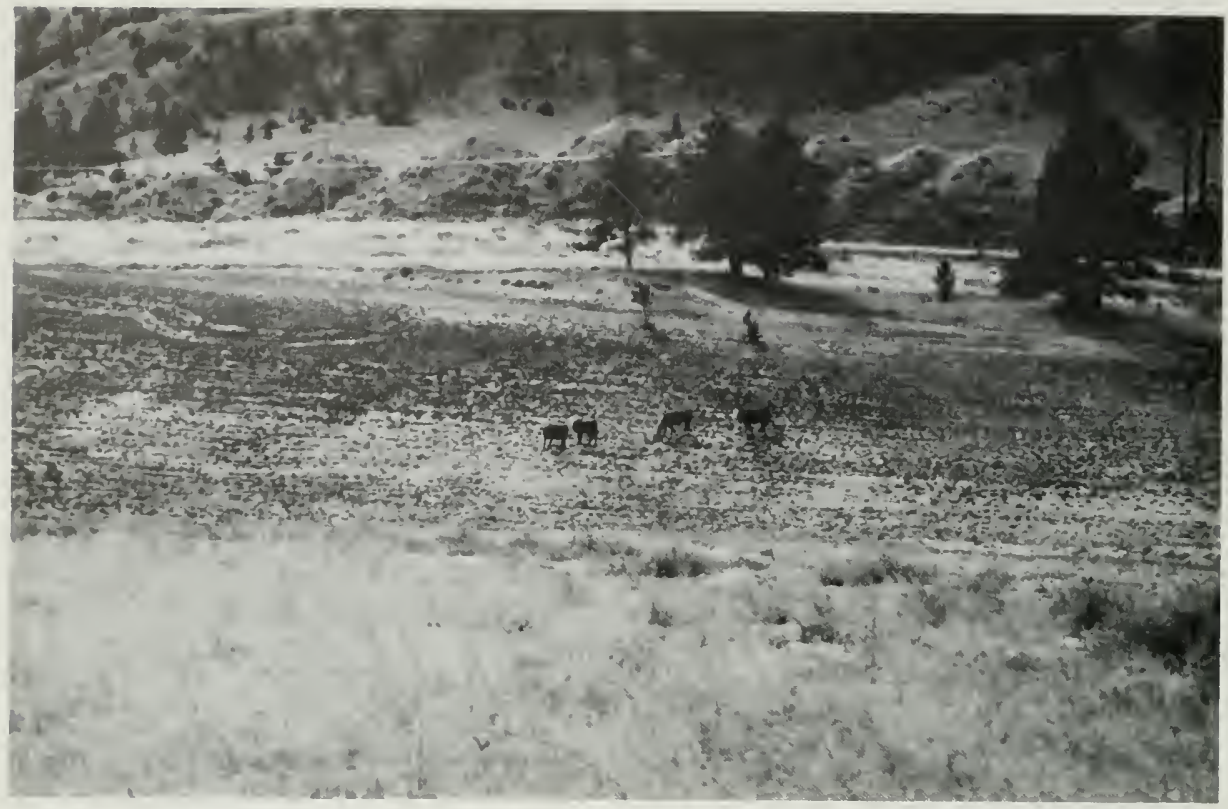

FIGURE 31.-Cattle grazing on young seedlings on a graded highwall with second-year grasses in foreground, at the Rosebud mine in Montana. Uncontrolled grazing by livestock, here unauthorized, can greatly reduce revegetation success and increase erosion potential. The natural vegetation is seen on the hillside in the background. Note the terrace-like trails made by livestock on the hillside (right). October 1978.

lands. On the basis of our 1978 examinations and the supporting data, we agree that there is a just cause for some of Wali and Kollman's optimism if present technology is applied; other factors, however, could preclude early success.

Ample evidence exists to show that grass or grass-legume cover can be initially established on gently sloping surface-mined land (figs. 39 , 40 , and 41 ) by relatively simple procedures and good post-planting protection. Density of the cover and overall plant productivity will, of course, vary with climatic conditions; reclaimed areas having the lowest annual precipitation (10 inches or less) and the highest evaporation rates (64 inches or more) will support less vegetation just as they did before mining. Within any given climatic zone, the better the solutions of problems related to soil, slope, moisture, and erosion, the better the stand of vegetation. The grasses and legumes planted in the successful projects were selected for their adaptability to and tolerance of the harsh conditions that generally prevail at a mine reclamation site. There is much less evidence of what other perennial plants can do in similar or less harsh situations, mainly because such plants have not been widely used. This situation seems to be caused by the small number of seed sources, the related high cost of seeds, and the lack of reliable performance data for native plants. Whereas nongrass herbaceous perennials and a few invasive shrubs are sometimes found within the grassplanted areas, they are not significant enough in number or size to enable predictions about their future competitive behavior. The voluntary occurrence of the nongrass and leguminous plants also has been cited as an indication that their intentional use in the reclamation process will be successful. Where they have been planted with grasses, however, the plantings are still too 


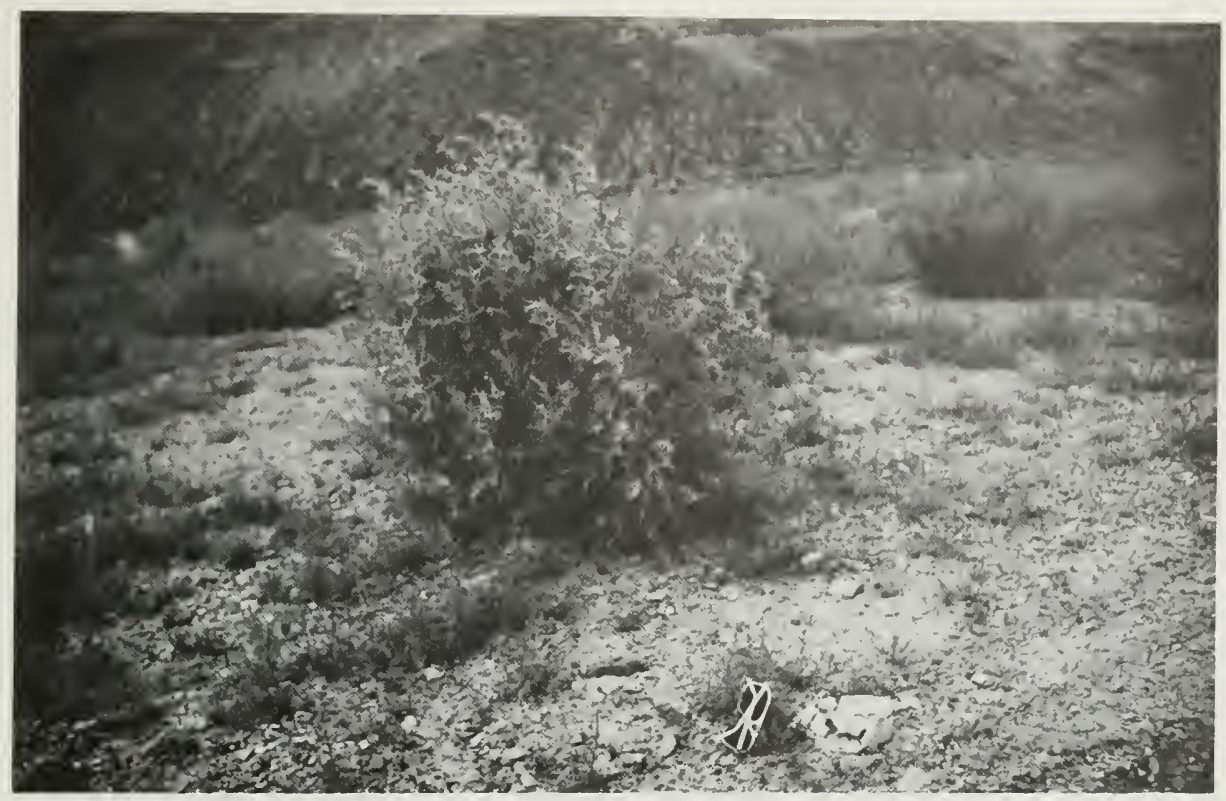

FIGURE 32.-Isolated 2- to 3-year-old fourwing saltbush plant on overgrazed reclaimed ground at the Black Mesa mine in Arizona. Most of the seeded vegetation was destroyed by overgrazing, and the visible plant life now is mostly the annual weed Russian-thistle. July 1978.

young to be evaluated. Furthermore, some native species have been found to be difficult to establish from seed.

The main question, however, is what will happen over long periods of time. Is it possible to predict the long-term vegetative productivity potentials? Can a self-sustaining diverse plant community composed primarily of native species be artificially reestablished? Only a few papers about western coal mining directly examine the time factor. Most reclamation research has focused on the how-to-do-it process with little or no emphasis on the long-term results of natural or deliberate revegetation. Successful long-term results are predicted largely on the basis of "effective" post-mining management (Hodder, 1978, p. 154). Moreover, organized plans for long-term monitoring and study of test plots are generally lacking except at a few places such as the West Decker mine in Montana (Richardson and others, 1975; B.Z. Richard- son, U.S. Forest Service, oral communication, 1980). Without such long-term monitoring, much valuable data may be lost. A more holistic research program, one that emphasizes the interrelationship between components and external factors affecting reclamation, is warranted.

\section{THE TEST OF TIME}

Although a growing body of information attests to the early increase of productivity in the first 4 to 5 years of revegetation, whether by natural succession (Schumacher and others, 1977 , p. 14) or by planting (John Lovell, oral communication, 1978; Packer and others, 1981, U.S. Geological Survey and Montana Department of State Lands, 1979, p. IV-34), this increase is followed by a leveling off or a decrease in plant growth and density. Packer, however, believes (oral communication, 1979) that what is seen at 


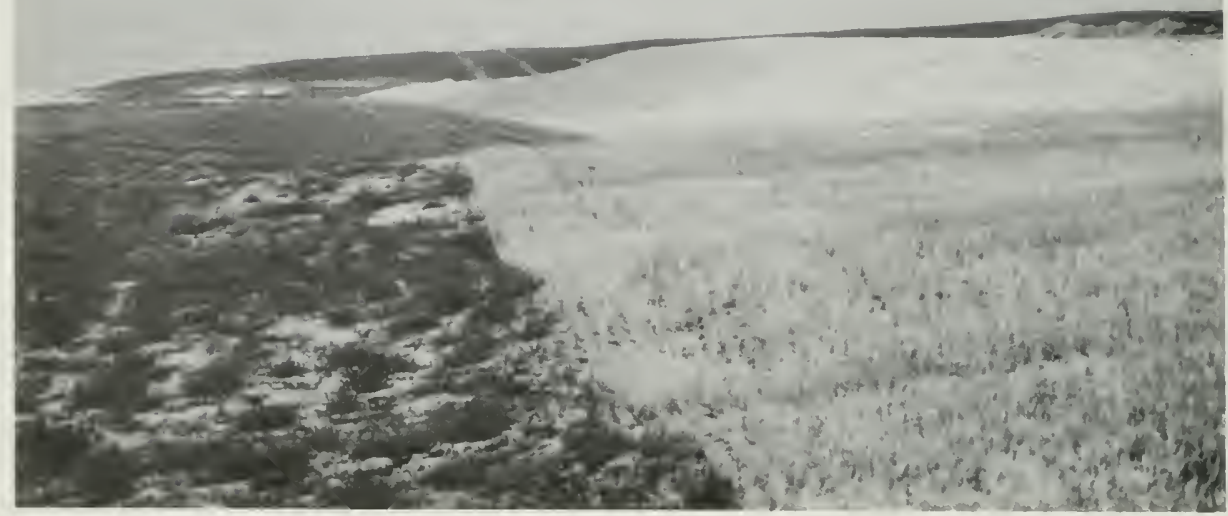

FigurE 33. - Surface-mined land in various stages of reclamation in the Dave Johnston mine in Wyoming. First-season growth (dark area, left foreground) consists mainly of Russian-thistle and includes small clumps of wheatgrass. Second-season growth (right foreground) consists of mature western and crested wheatgrasses. In the left center, a first-season millet cover crop protects part of the area while wheatgrasses and forbs become established. At the right center, smoothed sandy spoil was seeded but the seeding failed. The area in the background is covered by wheatgrasses and forbs in their third growing season. September 1975 .

the end of about 5 years is a measure of what the final productivity potential will be, assuming good management and non-drought conditions.

Information about natural revegetation in older mine areas is useful in estimating the potential effectiveness of reclamation efforts. Examination of abandoned old surfaces provides insights into how surfaces need to be modified to improve reclamation and what might happen if reclamation attempts fail. It also provides some time-related information on the evolution and stabilization of the plant communities.

The data of Schumacher and others (1977) on Canadian raw coal-mine spoils, which are under generally hospitable moisture and soil conditions, indicated a sharp decrease in productivity after 4 years. This situation continued for about 15 to 20 years before productivity increased again; after 30 years, productivity surpassed that of the first increase. They recognized five distinct vegetative growth stages upon reaching, at approximately 50 years, an "early" stage of the original aspen parkland vegetative type. These successive stages included changes in species dominance and in the total number of species, which changed from 14 species after 2 years, to 20 after 19 years, and to 50 after 31 years. At that time, 35 of the 50 species were nongrass herbaceous plants. In less hospitable areas, however, productivity may not increase at all. On coal-mine spoils in southeastern Saskatchewan, for example, Jonescu (1974) found conditions so unfavorable on the ridges that there were no significant differences in the vegetative cover on fresh spoils and on those 40 years old.

Wali and Freeman in a 1972-73 study (1973) compared soil characteristics and natural reveg- 


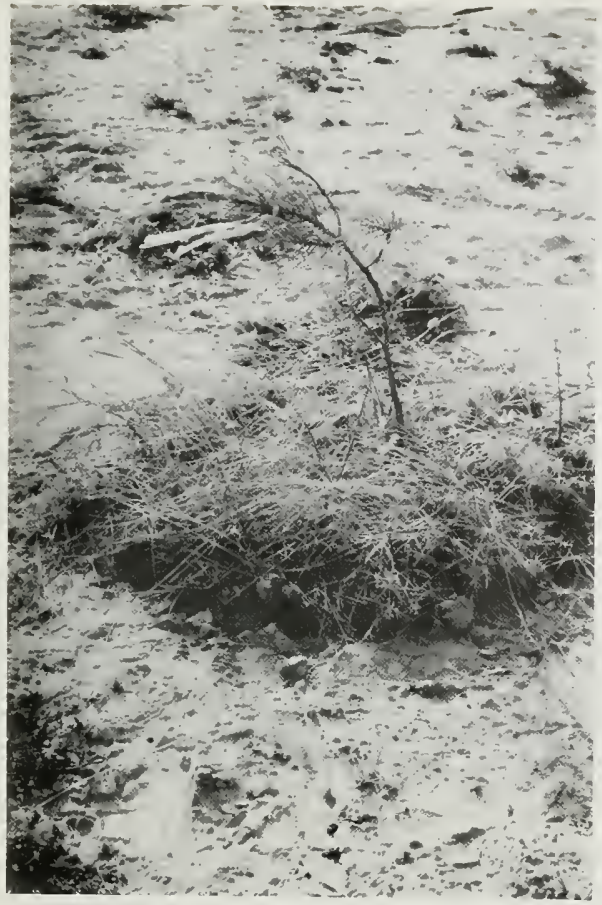

FIGURE 34.-Small native ponderosa pine trees were trans. planted in the spring of 1978 at the Absaloka mine in Montana. Some trees on this experimental planting area are over 10 feet tall and show normal growth. The patches of native grasses, forbs, and shrubs that were transplanted along with the trees are excellent sources of native plants. October 1978.

etation on five North Dakota lignite surface-mine spoils to the soils and vegetation on adjacent undisturbed land. They found about 50 perent fewer plant species on the mine spoils, which ranged in age from 1 to 53 years. Even on the 53-year-old spoils where the number of species was similar to that on the undisturbed lands, the plants showed less vigor and reduced growth rates. Many bare and sparsely vegetated areas remained.

About 1,000 miles south of the CanadianNorth Dakota study area, on a pinyon-juniperl shrubland complex in New Mexico, natural succession on coal-mine spoils ranging in age from
1 to 13 years was studied by Wagner and others (1978). At this semiarid site, no changes were observed over the whole 13-year vegetative sequence. Spoils of all ages showed only the very early stages of plant invasion; 19 to 34 species were present, but most were annuals with Russian-thistle still dominant. No correlation of the number of species present and the age of the spoils could be made. Spoils that covered the largest areas had the fewest plant species.

The replanted surfaces examined in this study are much younger than those examined in the above four studies. Because most of the planted sites have only a few years growth, prediction of their ultimate productivity is uncertain. Although not yet tested, the study by Packer and others (1981) will provide a predictive method for estimating the productivity of reclaimed lands. The time necessary for recovery for any targeted vegetation type could be reduced from that of natural succession rates by the deliberate introduction of a desired plant group or species or of a recognized successional series at an early stage. This time reduction has been noted by Curry (1975, p.40-41) along pipeline disturbances.

Something more than seed, water, and fertilizer is involved in making mine spoils productive with time. That something is undoubtedly the biological activity of the growing medium itself. A lengthy period of time is necessary in order to reinstitute and stabilize the general soil-forming process. Wali and Freeman (1973) concluded that 53 years was not time enough under natural conditions to make areas suitable for the growth of "desirable" plant species because there were still many significant physical and chemical differences between the soils present before and after mining. The time required should be significantly shortened by the replacement of topsoil and perhaps by the mixing of topsoil as an innoculant with surface spoils. What this topsoiling effect will be-relative to helping the planted surfaces to go through the normal sequential revegetation stages to a stable plant community -is still uncertain. Wali and Kollman $(1977$, p. 114) indicated that the vegetation could either be comparable to that developing on an abandoned field (the "old field" successional stage) or resemble that found when plants first invade an area with no soil development (the primary "soil forming" successional stage). 


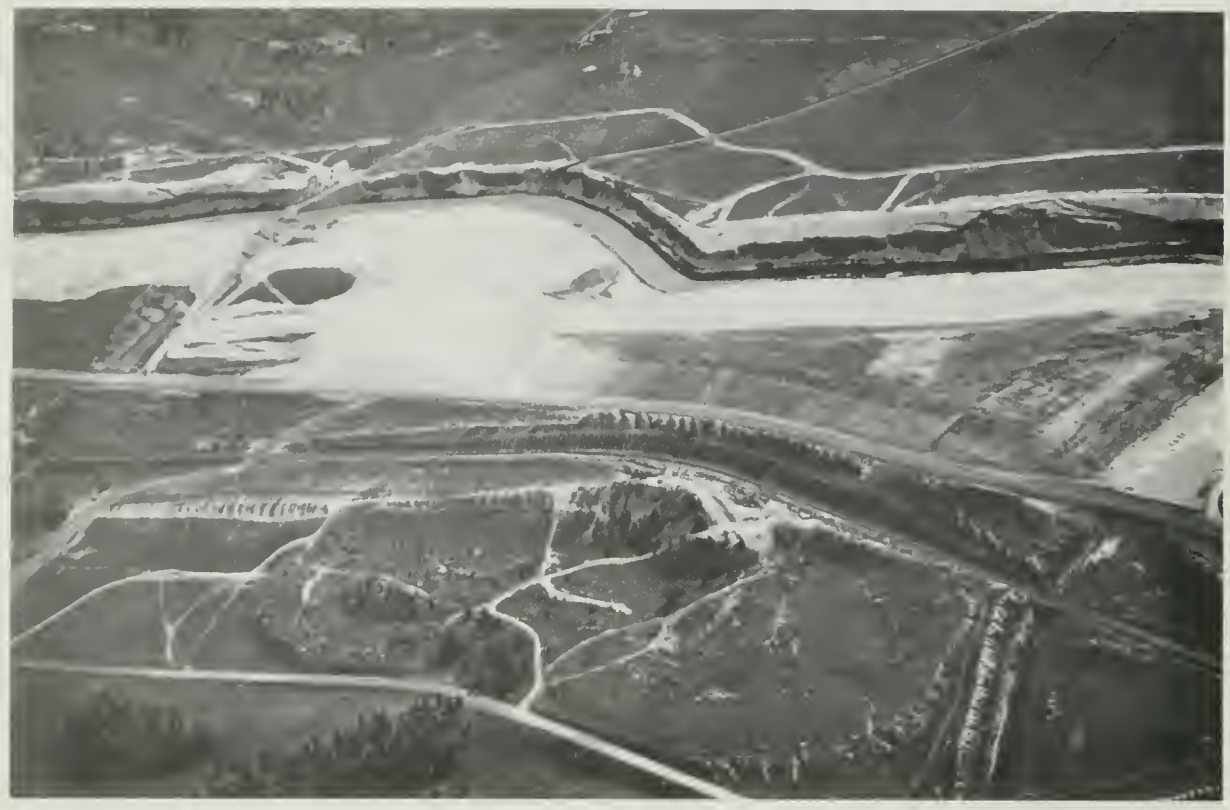

FIGURE 35.-Aerial photograph showing mining and reclamation sequence at the Rosebud mine in Montana. The natural landscape of grass and conifers is shown in the lower left and upper part. In the foreground, a former site for mine-associated buildings was voluntarily regraded and has partly naturally revegetated; the smooth spoil still shows the casting pattern which has limited revegetation. Recently reclaimed spoil (right center) was air seeded. Mining is proceeding toward the upper left. Haulways and final cuts, some partly refilled with smoothed spoil, contain impoundments of water. July 1978.

\section{SUMMARY}

After examining the mine sites, we concluded that the specific long-term results of reclamation cannot be predicted with certainty. We believe, however, that although general timetables are not now possible, guidelines do exist for determining the potential for reclamation success. In areas of higher moisture availability to plants and stable land surfaces, a gradual natural recolonization by diverse species was observed on raw spoils. We see no reason, therefore, why revegetation cannot occur under a planned reclamation program. In no place was climax vegetation reestablished; however, what appeared to be intermediate stages of succession were observed at some mine sites. For these higher moisture areas, we also expect that land productivity, however measured, can be eventually returned to nearly the original level for a soil of given character. The more the newly planted surface is used by man, his animals, and his vehicles, the longer the revegetation process will take; it could even be reversed under adverse conditions. For drier areas, we believe the same potentials exist, but we caution that these areas are much more sensitive to overuse.

\section{REFERENCES}

Aldon, E. F., 1975, Reclamation of coal-mined land in the southwest: Journal of Soil and Water Conservation, v. 33, no. 2, p. 75-77.

1978, Endomycorrhizae enhance shrub growth and survival on mine spoils, in Wright, R. A., ed., The reclamation of disturbed arid lands: Albuquerque, University of New Mexico Press, p. 174-179.

Aldon, E. F., Springfield, H. W., and Sowards, W. E., 1976, Demonstration test of two irrigation systerns for plant establishment on coal-mine spoils, in Fourth Symposium Surface mining and reclamation, NCANCR coal 


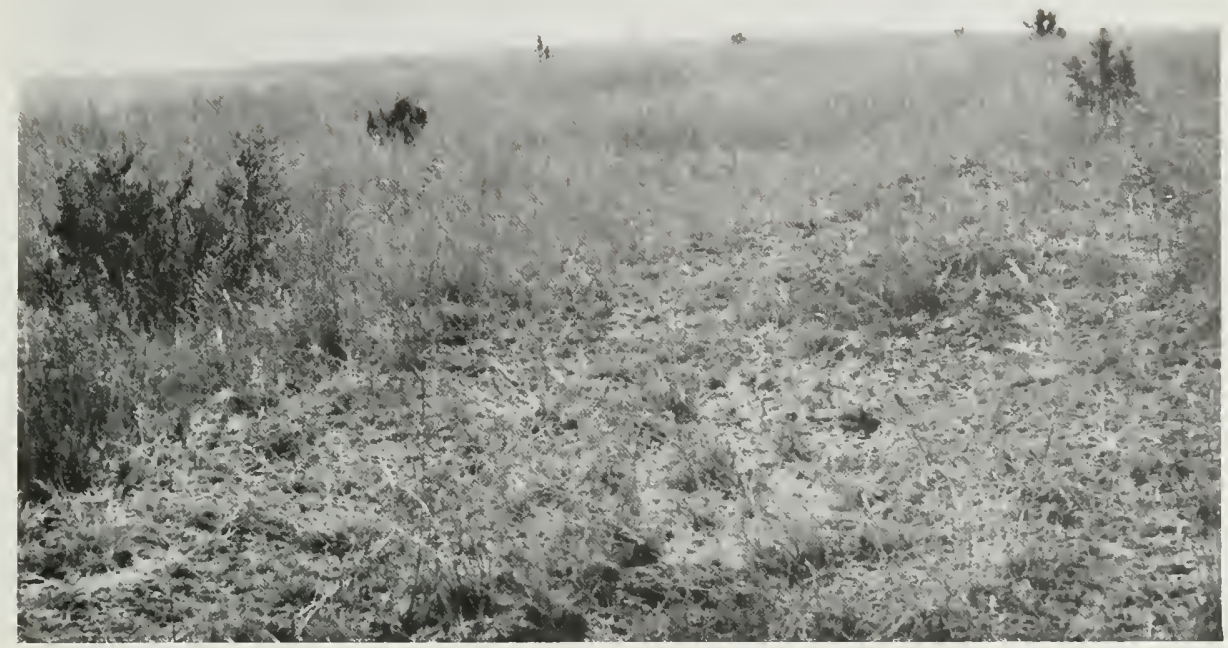

FIGURE 36. - Reclaimed area of wheatgrasses, pines, and junipers at the Big Sky mine in Montana. Six- to 15-foot ponderosa pines and Rocky Mountain junipers, normally difficult to transplant, are in their second year of growth after being transplanted into grass-covered reclaimed ground. The grasses are mainly western, crested, and thickspike wheatgrasses. October 1978.

conference and Expo III [Louisville, Kentucky, October 1976]: National Coal Association, Washington, D.C., p. 201-214.

Beauchamp, H., Lang, R., and May, M., 1975, Topsoil as a seed source for reseeding strip mine spoils: Laramie, University of Wyoming Agricultural Experimental Station Research Journal 90, $8 \mathrm{p}$.

Berg, W. A., 1975, Revegetation of land disturbed by surface mining in Colorado, in Wadi, M. K., ed., Practices and problems of land reclamation in western North America: Grand Forks, The University of North Dakota Press, p. 79-89.

Bjugstad, A. J., 1978, Reestablishment of woody plants on mine spoils and management of mine water impoundments: An overview of Forest Service research on the Northern High plains, in Wright, R. A., ed., Reclamation of disturbed arid lands: Albuquerque, University of New Mexico Press, p. 3-12.

Brown, D., 1977, Equipment for reclaiming strip-mined land: U.S. Forest Service Handbook 7728-2503, 58 p.

Burbank, F. M., 1978, Thirty-two years of cooperative range improvement and land reclamation in the U.S., in Canadian Land Reclamation Association, 3d annual meeting, Sudbury, Ontario, 1978, Proceedings, p. 258-265.

Cable, D. R., 1977, Western wheatgrass transplants grow well on raw mine spoil: U.S. Forest Service Research Note RN-345, p. 2.

Cook, C. W., Hyde, R. M., and Sims, P. L., 1974, Revegetation guidelines for surface-mined areas: Fort Collins, Colorado State University Range Science Series 16,73 p.

Crofts, K. A., and McKell, C. M., 1977, Sources of seeds and planting materials in the Western States for land rehabilitation projects: Logan, Utah State University Agricultural Experimental Station Land Rehabilitation Series $4,19 p$.

Cundell, A. M., 1977, The role of micro-organisms in the re vegetation of strip-mined land in the Western United States: Journal of Range Management, v. 30 , no. 4 , p. 299-305.

Curry, R. R., 1975, Biogeochemical limitations on western reclamation-the high Northern Great Plains example, in Wadi, M. K., ed., Practices and problems of land reclamation in western North America: Grand Forks, University of North Dakota Press, p. 18-47.

Deveraux, D. G., 1978, Reclamation at the Dave Johnston Mine, in Canadian Land Reclamation Association, 3d annual meeting, Sudbury, Ontario, 1978, Proceedings, p. 200-206.

Dollhopf, P. J., 1979, Characterization and selective placement of inhibitory material in Montana, in Canadian 


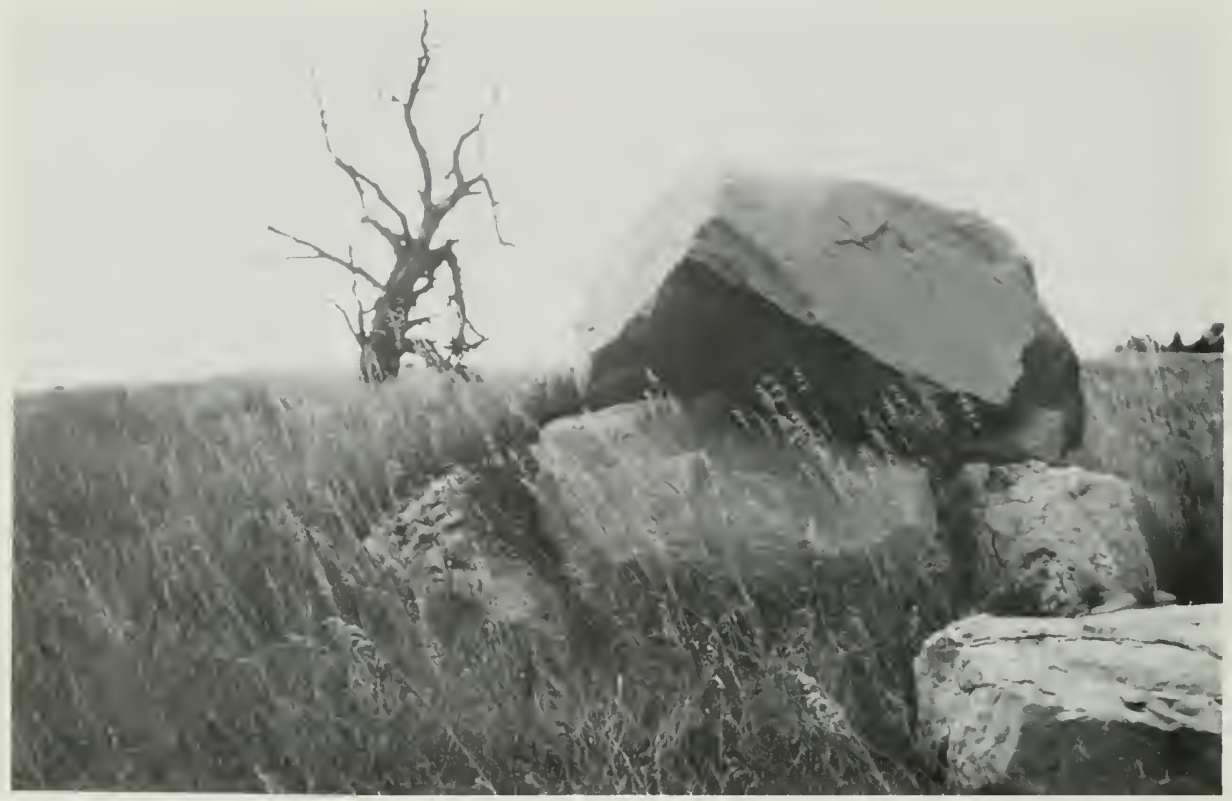

FIGURE 37.-Rocks and a dead tree that provide perches for hawks and owls on reclaimed area at the Rosebud mine in Montana. They were emplaced in 1973 by a frontend loader. The wheatgrasses and forbs are in their fifth growing season. Providing habitats for predators has reduced the amount of damage by rodents to the new plantings. October 1978 .

Land Reclamation Association, 4th annual meeting, Regina, Saskatchewan, 1979, Proceedings, p. 105-120.

Ettershank, G., Elkins, N. Z, Person, F. S., Whitford, W. G., and Aldon, E. F., 1978, The use of termites and other soil fauna to develop soils on strip-mine spoils: U.S. Forest Service Research Note RN-361, 4 p.

Evans, K. E., Uhleman, E. W., and Ebg, P. A., 1978, Atlas of western surface-mined lands: coal, uranium and phosphate: U.S. Fish and Wildlife Service, FWS/OBS-78/20, p. 302.

Frischknecht, N. C., 1978, Use of shrubs for mined land reclamation and wildlife habitat, in Workshop on reclamation for wildlife, Fort Collins, Colorado: U.S. Forest Service, Intermountain Forest and Range Experimental Station, Proceedings, $14 \mathrm{p}$.

Frischknecht, N. C., and Ferguson, R. B., 1979, Revegetating processed oil shale and coal spoils on semiarid lands: Prepared by U.S. Forest Service for the U.S. Environmental Protection Agency, EPA-600/7-79-068, $47 p$.

Gibson, D. F., Lehman, T. E., and Mooney, E. L, 1979, Computer aided design of surface coal mines: Bozeman, Montana State University, College of Engineering, p. $27-33$

Gillin, B., 1980, Comment letter number 24, in Northern Powder River Basin regional coal development final envi- ronmental statement: U.S. Geological Survey and Montana Department of State Lands, Final Environmental Statement 80-1.

Gleason, V. E., 1979, Bibliography on mined land, coal and the environment, abstract series: U.S. Environmental Protection Agency EPA-600/7-79-102, 373 p.

Hodder, R. L., 1978, Potentials and predictions concerning reclamation of semiarid mined lands, in Wright, $\mathrm{A}$. A., ed., Reclamation of disturbed arid lands: Albuquerque, University of New Mexico Press, p. 149-154.

Imhofi, E. A., Friz, T. O., and LaFevers, J. R., 1976, A guide to State programs for the reclamation of surface mined areas: U.S. Geological Survey Circular 731, 33 p.

Jensen, I. B., and Schafer, W. M., 1979, Effects of surface manipulation on percolation, infiltration and ground water quality, in Canadian Land Reclamation Association, 4th annual meeting, Regina, Saskatchewan, 1979, Proceedings, p. 121-137.

Jonescu, M. E., 1974, Natural vegetation and environmental aspects of stripmined land in the lignite coal fields of southeastern Saskatchewan: University of Saskatchewan, unpublished thesis, $234 \mathrm{p}$.

Kay, B. L., 1978, Mulch and chemical stabilizers for land reclamation in dry regions, in Schaller, F. W., and Sutton, P., eds., Reclamation of drastically disturbed lands: 


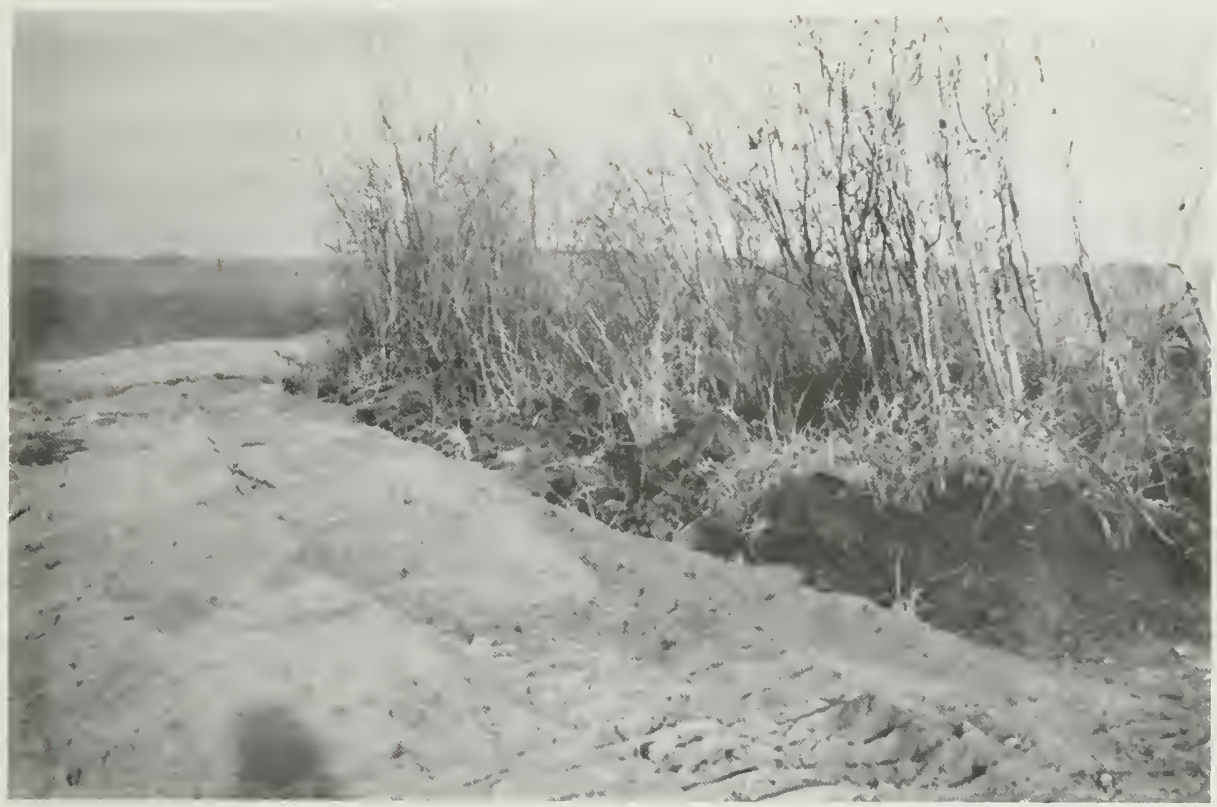

FIGURE 38. - Transplanted native aspens at Energy 1 mine in Colorado provide wildlife cover and sources of native plant seed in the reclaimed land. At this mine, a frontend loader is used to transport aspen, oak brush, and serviceberry scrub clumps from the areas being cleared to the reclaimed areas. Clumps are placed on leveled spoil, and topsoil is then spread around the clumps. Piles of rock are also placed randomly on smoothed spoil prior to topsoiling to provide small animal habitat. November 1978.

Madison, Wisconsin, American Society of AgronomyCrop Science Society of America-Soil Science Society of America, p. 467-483.

Lindsey, D. L., Cress, W. A., and Aldon, E. F., 1977, The eftects of endomycorrhizae on growth of rabbitbrush, fourwing saltbush, and corn on coal mine spoil material: U.S. Forest Service Research Note RN-343, 6 p.

Monsen, S. B., and Plummer, A. P., 1978, Plants and treat ment for revegetation of disturbed sites in the intermountain area, in Wright, R. A., ed., Reclamation of disturbed arid lands: Albuquerque, University of New Mexico Press, p. 155-173.

National Academy of Sciences, Environmental Studies Board, 1974, Rehabilitation potential of western coal lands: Cambridge, Massachusetts, Ballinger Publishing Co., $198 \mathrm{p}$.

Nobel, E. L., 1978a, Reclamation potential of selected strip coal mines in Montana, North Dakota, and Wyoming: U.S. Geological Survey, contractor report, $30 \mathrm{p}$.

1978b, Reclamation potential of selected strip coal mines in Arizona, Colorado, and New Mexico: U.S. Geological Survey, contractor report, $18 \mathrm{p}$.
Packer, P. E., and Aldon, E. F., 1978, Revegetation techniques for dry regions, in Schaller, F. W., and Sutton, P., eds., Reclamation of drastically disturbed lands: Madison, Wisconsin, American Society of Agronomy-Crop Science Society of America-Soil Science Society of America, p. 425-450.

Packer, P. E., Jensen, C. E., Noble, E. L., Marshall, J. A., 1981, A model to estimate revegetation potentials of land surfaces mined for coal in the west: U.S. Forest Service General Technical Report.

Plummer, A. P., 1977, Revegetaton of disturbed intermountain area sites, in Thames, J. L., ed., Reclamation and use of disturbed land in the southwest: Tuscon, The University of Arizona Press, p. 302-339.

Pole, M. W., Bauer, Armand, Zimmerman, LeRoy, and Melstad, S. W., 1979, Effects of topsoil thickness placed on spoilbanks on wheat and corn yields in North Dakota, in Canadian Land Reclamation Association, 4th annual meeting, Regina, Saskatchewan, 1979, Proceedings, p. 139-155.

Power, J. F., 1978, Reclamation research on strip-mined lands in dry regions, in Schaller, F. W., and Sutton, P., eds., 


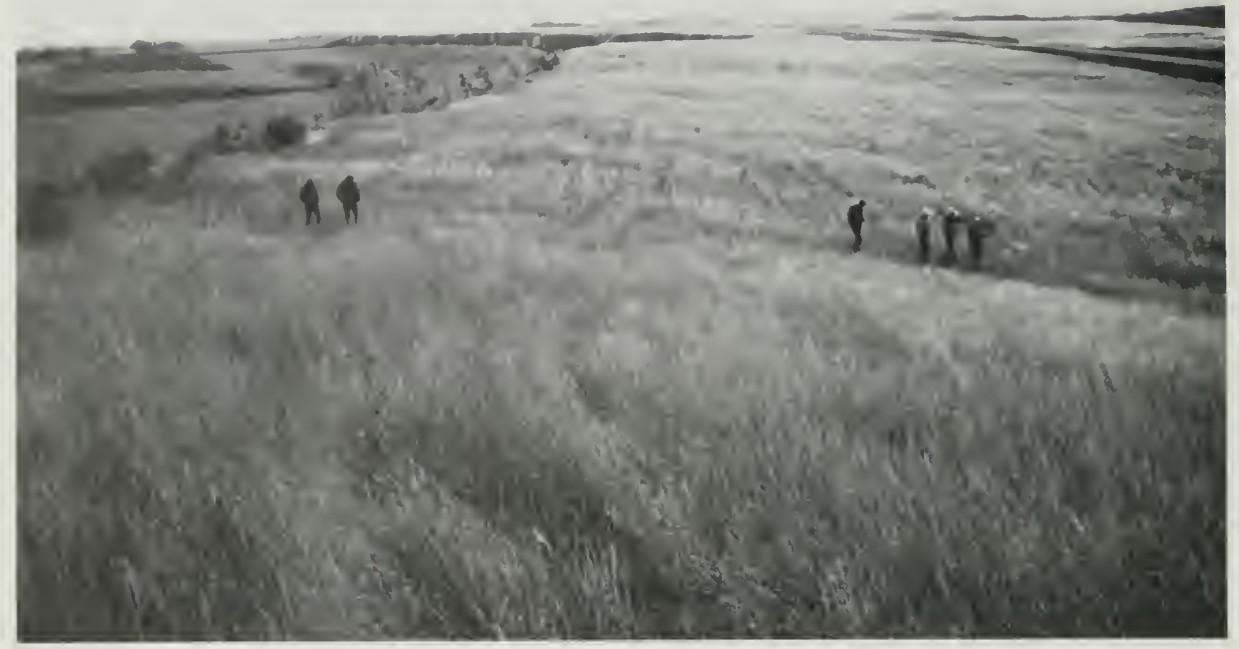

FIGURE 39.-Lush grasses on reclaimed land at the south Beulah mine in North Dakota. Species include western and intermediate wheatgrasses, brome, and alfalfa. The escarpment on the left is lined with cottonwoods, willows, and other deciduous trees and marks the edge of natural vegetation along the initial cut. Mining proceeded from left to right. Gentle slopes and excellent reclamation practices in an area of relatively high moisture (16 to 18 inches of rainfall per year), and low evapotranspiration have produced this dense vegetative cover. October 1978.

Reclamation of drastically disturbed lands: Madison, Wisconsin, American Society of Agronomy-Crop Science Society of America-Soil Science Society of America, p. 521-535.

Richardson, B. Z., Farmer, E. E., Brown, R. W., and Packer, P. E., 1975, Rehabilitation research and its application on surface mined areas of eastern Montana, in Proceedings ot the Fort Union Coal Field Symposium with Montana Academy of Science, v. 3, Reclamation Section: Billings, East Montana College, p. 247-265.

Ries, R. E., and Day, A. D., 1978, Use of irrigation in reclamation in dry regions, in Schaller, F. W., and Sutton, P., eds., Reclamation of drastically disturbed lands: Madison, Wisconsin, American Society of Agronomy-Crop Science Society of America-Soil Science Society of America, p. 505-520.

Sandoval, F. M., and Gould, W. L, 1978, in Schaller, F. W., and Sutton, P., eds., Reclamation of drastically disturbed lands: Madison, Wisconsin, American Society of Agronomy-Crop Science Society of America-Soil Science Society of America, p. 485-504.

Schumacher, A. E., Hermish, R., and Bedwang, A. L., 1977, Coal mine spoils and their revegetation patterns in central Alberta, in Canadian Land Reclamation Association, 2d annual meeting, Edmonton, University of Alberta, 1977,20 p.

Scholz, K. E., 1978, SEAM report for FY 1977: U.S. Forest Service, Surface Environment and Mining Program, $18 \mathrm{p}$.

Scott, M. D., 1978, Use of the computer for reclamation planning at large surface mines, in Canadian Land Reclamation Association, 3d annual meeting, Sudbury, Ontario, 1978, Proceedings, p. 309-314.

Thornburg, A. A., 1980, Plant materials for use on surfacemined lands in arid and semiarid regions: U.S. Soil Conservation Service.

Thornburg, A. A., and Fuchs, S. H. 1978, Plant materials and requirements for growth in dry regions, in Schaller, F.W., and Sutton, P., eds., Reclamation of drastically disturbed lands: Madison, Wisconsin, American Society of Agronomy-Crop Science Society of America-Soil Science Society of America, p. 411-423.

Trimble, D. F., 1979, Unstable ground in western North Dakota: U.S. Geological Survey Circular 226, $19 p$.

U.S. Geological Survey and Montana Department of State Lands, 1979, Draft environmental statement, Northern Powder River Basin coal, Montana: U.S. Geological Survey and Montana Department of State Lands, Draft 


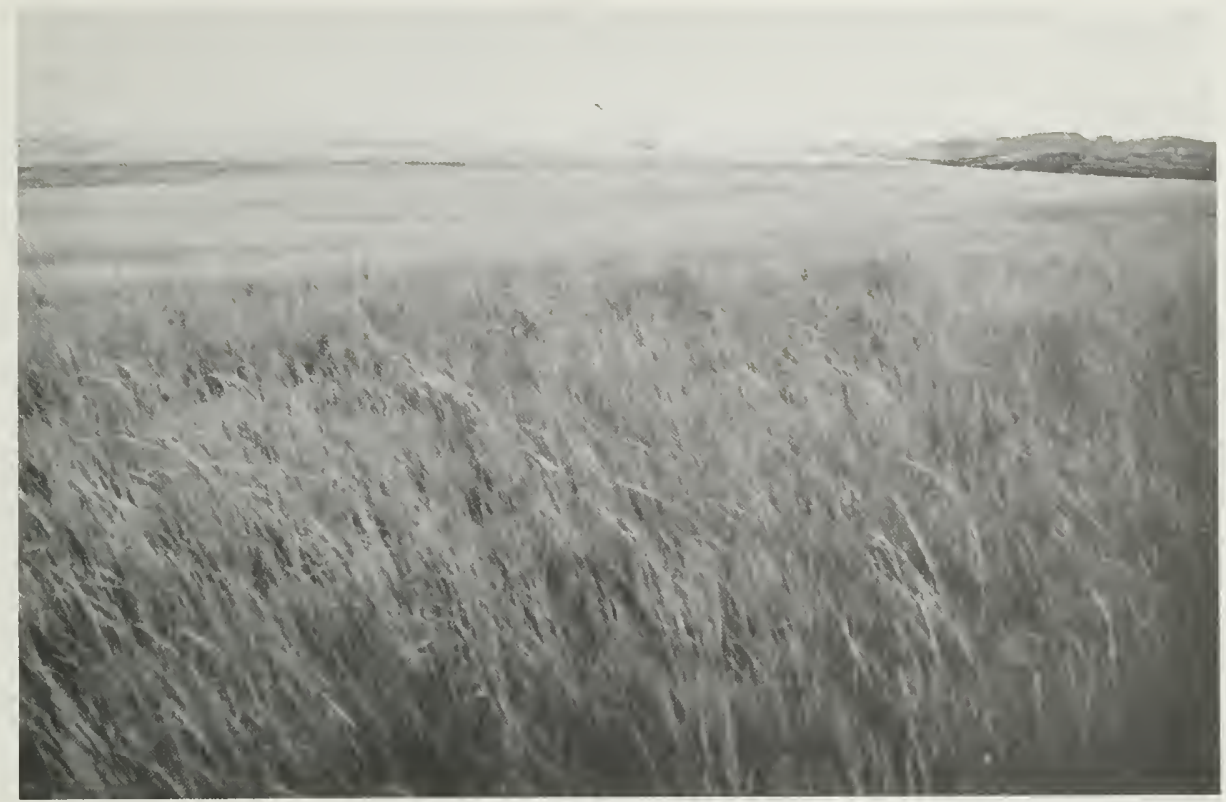

FIGURE 40.- Reclaimed land covered by grasses in their third growing season at the West Decker mine in Montana. Mining operations are continuing in the right background. August 1978.

Environmental Statement 79-41, v. I, Regional Analysis, $315 \mathrm{p}$.

U.S. Office of Surface Mining Reclamation and Enforcement, 1979, Surface coal mining and reclamation operations permanent regulatory program: Federal Register, v. 44, no. 50, p. 15311-15463.

Wagner, W. L., Martin, W. C., and Aldon, E. F., 1978, Natural succession on strip-mined lands in northwestern New Mexico: Pergamon Press, Reclamation Review, v. 1, p. 67-73.

Wali, M. K., and Freeman, P. G., 1973, Ecology of some mined areas in North Dakota, in Wali, M. K., ed., Some environmental aspects of strip mining in North Dakota: North Dakota Geological Survey Educational Series 5, p. 25-47.
Wali, M. K., and Kollman, A. L, 1977, Ecology and mining or mining ecology? in Thames, J. L., ed., Reclamation and use of disturbed land in the southwest: Tucson, The University of Arizona Press, p. 108-115.

Wyatt, J. W., Dollhopf, F. J., and Schafer, W. M., 1980, Root distribution in 1- to 48-year-old strip-mine spoils in southeastern Montana: Journal of Range Management, v. 33 , no. 2 , p. 101-104.

Williams, S. E., and Aldon, E. F., 1976, Endomycorrhizal associations of some arid zone shrubs: The Southwestern Naturalist, v. 20 , no. 4, p. 437-444.

Zak, B., 1975, Mycorrhizae and container seedlings, in 23d Western International Forest Disease Annual Work Conference Proceedings, p. 21-23. 


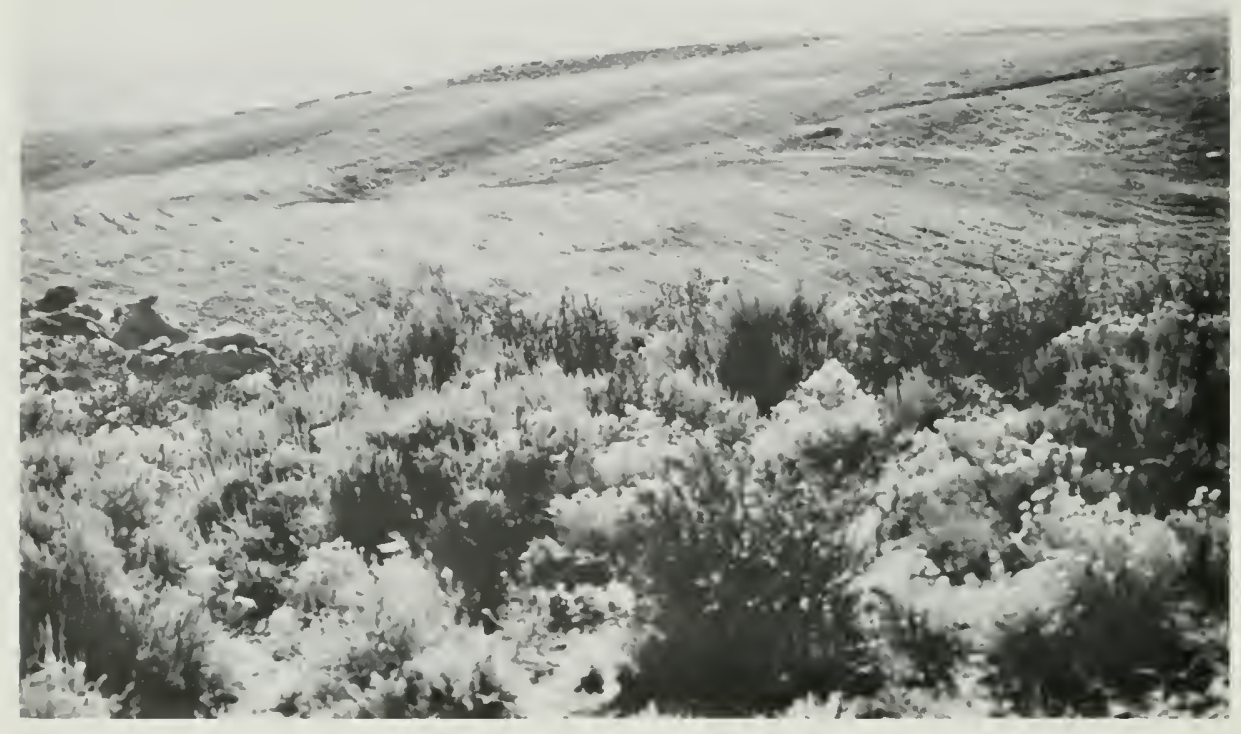

Figure 41.-Grass-seeded reclaimed land (middleground) blending into undisturbed land at the Vanguard mine in Wyoming. The natural undisturbed vegetation in the foreground has a rich diversity of shrub and herbaceous plant species but few grass species. It is heavily browsed but maintains its vigor. Although bare soil is seen between plants, evidence of erosion is slight. In the reclaimed area, the spoil was smoothed, topsoiled, and chisel plowed, and trenching was performed parallel to contours. October 1978. 


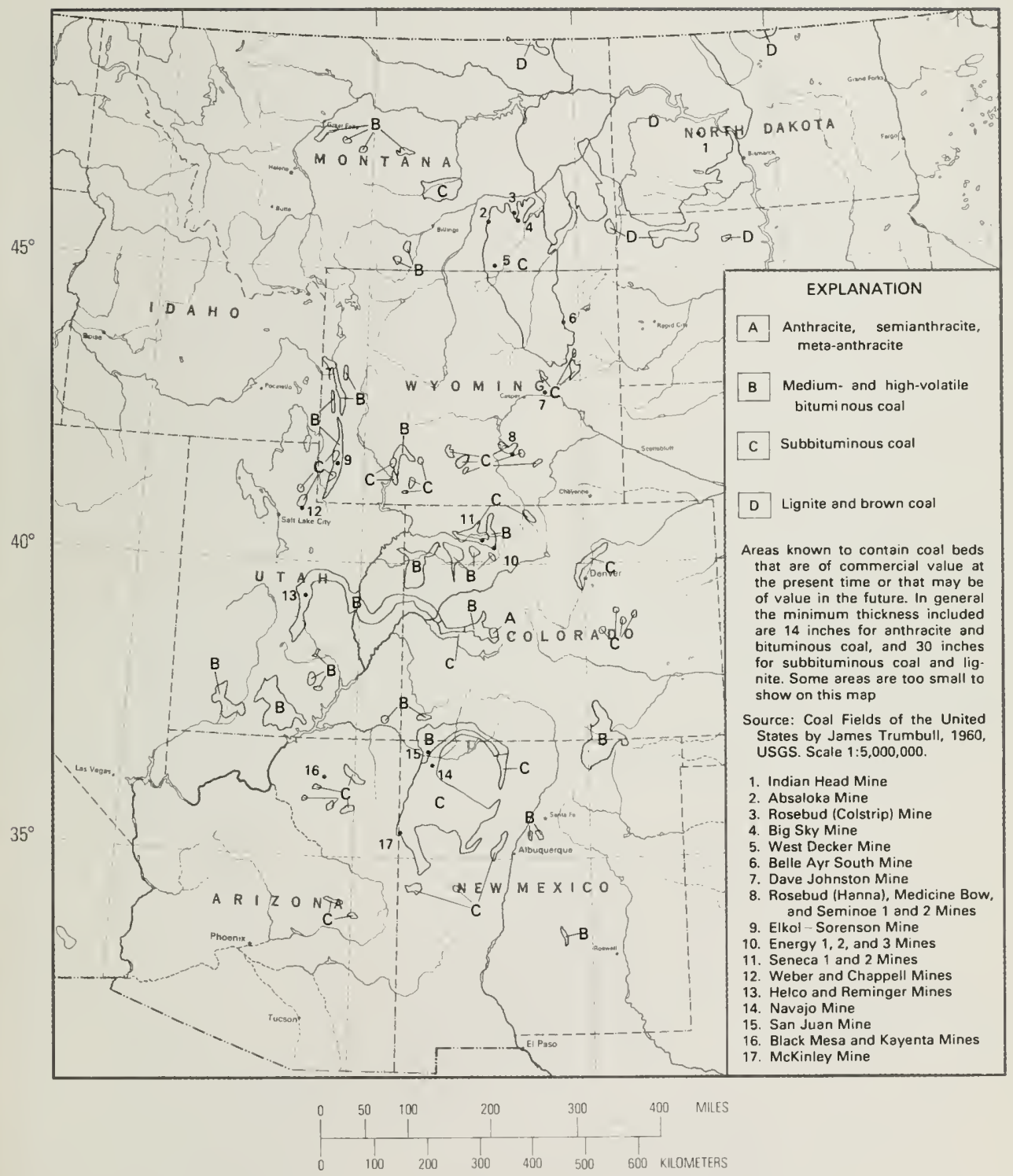



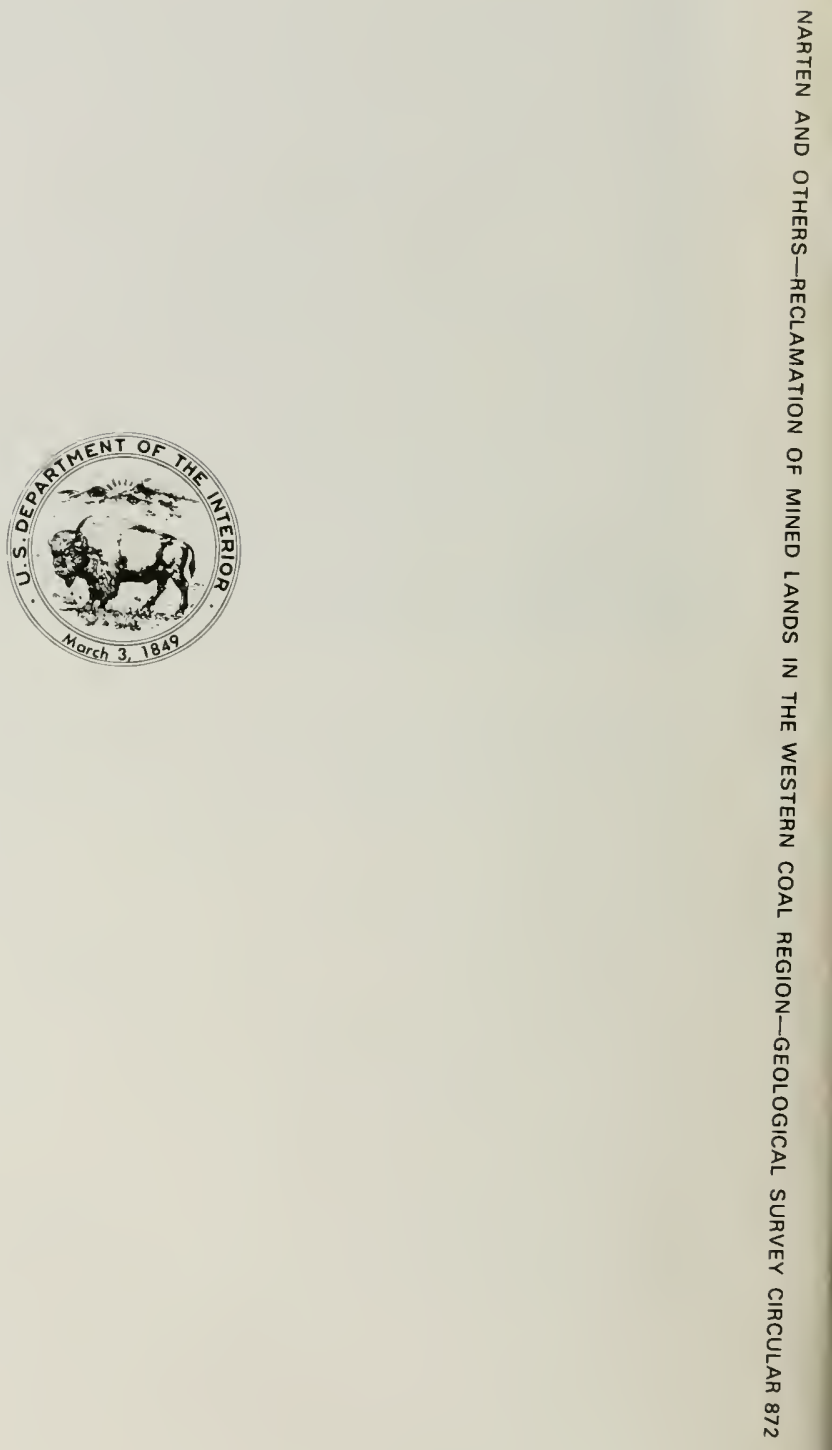